\title{
Does competence predict performance? : standardized patients as a means to investigate the relationship between competence and performance of general practitioners
}

Citation for published version (APA):

Rethans, J. J. (1991). Does competence predict performance? : standardized patients as a means to investigate the relationship between competence and performance of general practitioners. [Doctoral Thesis, Maastricht University]. Rijksuniversiteit Limburg. https://doi.org/10.26481/dis.19911031jr

Document status and date:

Published: 01/01/1991

DOI:

10.26481/dis.19911031jr

Document Version:

Publisher's PDF, also known as Version of record

Please check the document version of this publication:

- A submitted manuscript is the version of the article upon submission and before peer-review. There can be important differences between the submitted version and the official published version of record. People interested in the research are advised to contact the author for the final version of the publication, or visit the DOI to the publisher's website.

- The final author version and the galley proof are versions of the publication after peer review.

- The final published version features the final layout of the paper including the volume, issue and page numbers.

Link to publication

\footnotetext{
General rights rights.

- You may freely distribute the URL identifying the publication in the public portal. please follow below link for the End User Agreement:

www.umlib.nl/taverne-license

Take down policy

If you believe that this document breaches copyright please contact us at:

repository@maastrichtuniversity.nl

providing details and we will investigate your claim.
}

Copyright and moral rights for the publications made accessible in the public portal are retained by the authors and/or other copyright owners and it is a condition of accessing publications that users recognise and abide by the legal requirements associated with these

- Users may download and print one copy of any publication from the public portal for the purpose of private study or research.

- You may not further distribute the material or use it for any profit-making activity or commercial gain

If the publication is distributed under the terms of Article 25fa of the Dutch Copyright Act, indicated by the "Taverne" license above, 


\section{DOES COMPETENCE PREDICT PERFORMANCE?}

Standardized patients as a means to investigate the relationship between competence and performance of general practitioners 
5 


\section{DOES COMPETENCE PREDICT PERFORMANCE?}

Standardized patients as a means to investigate the relationship between competence and performance of general practitioners

\section{PROEFSCHRIFT}

ter verkrijging van de graad van doctor aan de Rijksuniversiteit Limburg te Maastricht, op gezag van de Rector Magnificus, Prof. mr. M.J. Cohen, volgens het besluit van het College van Dekanen, in het openbaar te verdedigen op donderdag, 31 oktober 1991 om 14.00 uur

door

Jan-Joost Rethans 
Promotoren: Prof. Dr. F. Sturmans

Prof. Dr. M.J. Drop

Co-promotor: Dr. C.P.M. van der Vleuten

Beoordelingscommissie:

Prof. Dr. C.P.A. van Boven, voorzitter

Prof. Dr. J.A. Knottnerus

Dr. J.C.M. Metz

Prof. Dr. H.G. Schmidt

Prof. Dr. Th. B. Voorn

Publication of this thesis was supported by a grant from E. Merck Nederland B.V. 
Ik bestudeerde de kaart (touwtje langs de wegen, vermenigvuldigen met de schaal), ik reed het parkoers met mijn auto, met de auto van een vriend, ik monteerde een kilometerteller, maar al die metingen gaven een andere uitkomst; het te meten object had alleen maar de gebrekkigheid van mijn meetmethodes aan de kaak gesteld.

I studied the map (stretching a piece of string along the roads and multiplying according to the scale), I drove the course in my own car and in a friend's; I fitted a mileometer. But all these measurements gave different results; the object to be measured had only served to expose the imperfections of my measuring methods. ....

Tim Krabbé in his book "De Renner"

Erven Thomas Rap, Vijverhof, Baarn, 1980, p. 39 
* 


\section{Preface}

This book originated as spin-off from one of my hobbies, namely longdistance running.

While running I get ideas, which usually tend to come at random or to relate to the persons and things I pass.

Sometimes, however, it is possible to concentrate on one particular issue.

During one of my training runs I was thinking about methods to open up the general practitioner's "black box", his/her consulting room.

One of the brainwaves which occurred to me was to get information about consultations by using simulated patients, who were to be sent to doctors" practices without the doctors knowing.

At first I laughed at the idea and rejected it as funny, not serious, and certainly not feasible.

However, the thought persisted and I decided to accept the challenge to start working with simulated (or standardized) patients in real practice.

It is therefore with the greatest pleasure that I am able to present this book. It is my hope that those who read it will enjoy it and will pick up at least one general idea: conducting research can be great fun! 



\section{Contents}

Chapter 1: Introduction

Chapter 2: Simulated patients in general practice: a different look at the consultation

Chapter 3: De dokter onderzocht: simulatiepatienten met mictieklachten op het spreekuur van de huisarts (The doctor examined. Simulated patients presenting micturition complaints in office encounters)

Chapter 4: Simulatiepatienten in onderwijs en praktijk, een literatuur overzicht (Simulated patients in medical education and medical practice. A literature survey)

Chapter 5: Competence and performance: two different concepts in the assessment of quality of medical care

Chapter 6: A method for introducing standardized patients into general practice consultations

Chapter 7: Assessment of the performance of general practitioners by the use of standardized patients

Chapter 8: Does competence of physicians predict their performance?

Chapter 9: Conclusions and recommendations for research

Summary

Samenvatting

Resymể på. Norsk

Thanks

Curriculum vitae

Appendices:

Detection form for the physicians to report standardized patients

Instructions for the participating general

practitioners (competence setting)

The standards of care used 

Chapter 1: Introduction 
This book contains the results, in the form of several papers, of two research projects conducted consecutively by the Department of General Practice at the University of Limburg in Maastricht.

The projects had two main aims. The first of these was to investigate whether it was possible for a set of standardized (or simulated) patients to visit general practitioners during normal surgery hours without being detected. The second aim was to investigate 1) the difference and 2) the relationship between competence and performance of general practitioners.

In this chapter both topics, the method of using standardized patients and the competence and performance of physicians, will be briefly introduced.

\section{The methodology of using standardized patients}

The immediate instigation for the use of standardized patients in real practice in the projects described here was a sentence in a medical paper. ${ }^{1}$ This referred to the hypothesis that there might be a discrepancy between what doctors actually do in their practice and what they say they do in an interview or in a questionnaire.

To measure what goes on in real practice requires instruments, which need to be valid and consistent. One of the best instruments in this regard is the use of standardized (or simulated) patients. ${ }^{*}$ Numerous studies have been published dealing with the use of this method for educational and licensing purposes in medical schools. In a recent review of the use of standardized patients, evidence is given for the validity and consistency of the method. ${ }^{2}$ Only a few studies have been published of the use of standardized patients in actual practice. ${ }^{3-10}$

The use of standardized patients in real practice has received much criticism in medical joumals. $11-15$ This criticism focussed mainly on the fact that the consent of the participating physicians was not obtained and on the lack of consistency (reliability) of the patients used.

A very strong point with regard to the use of standardized patients in real practice is that this makes it possible to compare doctors in their care for

\footnotetext{
- It was the neurologist Barrows who was the first to speak of and report on simulated patients. (Barrows H.S. The programmed patient: a technique for appraising student performance in clinical neurology. J Med Educ 1964; 39: 802-5). He discussed so called "programmed patients", meaning laymen and womern who had been trained to play a role as a patient in a confrontation with medical students or physicians. In 1971 Barrows changed the term "programmed patients" into "simulated patients" (Barrows HS. Simulated patients. Springfield (111.): Thomas, 1971). Through the years other names have been used, such as "pseudopatients" or "surrogate patients". Since 1987 there has more or less been a consensus to use the term "standardized patients" (Barrows HS. Simulated (standardized) patients and other human simulations. Chapel Hill (North Carolina): Health Sciences Consortium, 1987).
} 
patients, with the standardized patient as the independent variable and the doctors as the dependent one.

The lack of experience with this method in actual practice was one of the challenges to be met in the projects described here. With respect to this, the objective of the studies in this book was to investigate whether the method could be used in actual practice in a valid and consistent way. In this respect, the projects can be described as innovative and risky. This method made it possible to investigate the hypothesis that there might be a discrepancy between what doctors actually do in their practice and what they say they do in an interview, questionnaire or a paper-and-pencil test (first project, chapter two).

\section{Competence and performance}

The assessment of people's achievements is not only interesting in the area of sports, but is an even more serious business in the academic world of medicine.

Before a medical student can start a career as a medical doctor, she or he has to pass the necessary examinations. When this has been done one can speak of a competent doctor.

But does the passing of examinations tell us anything about the future performance of doctors? Intuition suggests that this should be the case but, surprisingly, no evidence exists to validate this assumption. Most of the studies intended to investigate the relationship between pre-examination and post-examination performance show a lack of sound methodological criteria. 16 For example, they either do not define the setting in which the participating physicians were assessed during the studies or they compare results collected with different instruments in the two settings.

A study was therefore needed which would investigate the relationship between competence and performance in a methodologically sound way.

Unfortunately, the literature on 'competence' makes use of an extensive vocabulary. Ability, behaviour, clinical judgment, clinical reasoning, habitual performance, problem solving, clinical competence, the combination of knowledge, skills and attitudes, naturalistic behaviour, medical competence, adequate performance, efficient performance, interpretive skills, optimal behaviour, criteria setting and clinical decision making are terms used (generally without definition) to describe this domain.

In this book the following definitions are used: performance is "what a doctor does in his day-to-day practice" and competence is "what a doctor is capable of doing". 17,18 There were several reasons for choosing these definitions.

First, they are mutually exclusive: they do not overlap. Second, the definitions leave the readers free to choose the type of measuring instruments 
with which to assess aspects of either competence or performance. That is, the two definitions apply to the settings of the doctors' professional activities and not to the domain of expertise. One might argue that competence is made up of several aspects and that it would be more appropriate to speak of 'competences', referring to competences as a series of parameters such as diagnostic and therapeutic skills, interpersonal skills, etc. In this book however we want to emphasize the concept of competence as a single entity. With the measurement of competence, or performance, both concepts can be operationalized into several aspects.

Third, both definitions appeal to the intuitive feeling that working in actual practice is different from working under examination conditions and for that reason are easy to understand.

\section{Standards of "care}

Besides the lack of methodologically sound studies with which to investigate the relationship between competence and performance, there is another reason why the studies described here were initiated. There have been a number of studies, showing that doctors in real practice perform below established standards of care. $9,10,19-21$

An interesting phenomenon in these studies is that it appears to make no difference whether the standards are made by colleagues, experts or even by the participating doctors themselves. That is, below-standard performance seems to be a consistent finding, regardless of the origin of the criteria. This was elegantly demonstrated by Norman, who asked physicians to construct standards of care which were (and this was stressed to the physicians) meant for practical use in actual care. ${ }^{9}$ After doing so, all the participating physicians were visited in their practice by standardized patients. The results of the study showed that the doctors as a group performed only $56 \%$ of the actions considered to be essential according to the standard.

This observation of below-standard performance by doctors has in the past led to action being taken by various professional organisations. This action was mostly directed towards mandatory attendance at continuing medical. education. The underlying assumption of such directives is that poor performance is a reflection of inadequate knowledge and/or skills, which are assumed to be capable of being remedied by additional instruction.

Studies regarding the effects of postgraduate education on the actual practice behaviour of doctors often produce conflicting evidence. 22 It has been shown, for instance, that additional postgraduate education does not seem to change the practice behaviour of doctors. ${ }^{23}$ A recent study concerning postgraduate teaching of fundoscopy to general practitioners showed that there was no measurable learning effect, although the doctors were very enthusiastic. ${ }^{24}$ In a study whose purpose was to describe the effects of training general practitioners in the application of standards, Grol showed 
that the participating physicians performed $51 \%$ of necessary actions after the project in contrast with $45 \%$ beforehand. 20 As a side effect of this project, however, doctors also performed more intermediate and superfluous actions, whereas it was expected that the incidence of such actions would be lowered after the project.

If, however, doctors do not lack competence, then the process of setting standards can also be questioned. This issue is interesting, since in recent years there has been a movement in general practice towards the designing of standards of care for practical purposes.

The designers of these standards seem to be convinced that by producing standards the quality of care in actual practice can be raised. With that in mind, the studies in this book also attempt to determine whether doctors can do better in a test situation, according to established consensus standards, than in their practical work. If doctors can in fact do better, it would be interesting to see whether they perform in line with standards or not.

\section{Structure of this book}

As mentioned before two studies with standardized patients have been conducted. The first study was conducted in 1986 when 48 general practitioners were consulted by standardized patients (chapters two and three). In 1989 a second study was conducted during which 39 general practitioners were consulted by standardized patients (chapters seven and eight).

Since the book is divided in chapters, which are based on papers, it is possible for the reader to read only the chapter or chapters which seem to be of interest. If one is interested enough to read all the papers, one will inevitably find some overlap between some of them.

Two of the papers (chapter 3 and 4) were written especially for Dutch readers and were published in Dutch medical journals. However, chapter 3 contains a extended summary in English and for chapter 4 (with an abstract in English) several alternative papers exist in English.2, 25.

Chapter 2 (Simulated patients in general practice: a different look at the consultation) contains the results of our first study with standardized patients. The purpose of this paper is to compare the actual performance of doctors ("what the doctor does") with answers with regard to a written case of a standardized patient ("what the doctor says he would do").

Chapter 3 (De dokter onderzocht /The doctor examinated. Simulated patients presenting micturition complaints in office encounters) contains results from the same study as in chapter 2 , now focussing on the feasibility of sending standardized patients into general practitioners' offices. 
Chapter 4 (Simulatiepatienten in onderwijs en praktijk, een literatuur overzich/ Simulated patients in medical education and medical practice) is a review paper, focussing on establishing the purposes for which standardized patients have been used and examining the reliability and validity of the use of standardized patients.

Chapter 5 (Competence and performance: two different concepts in the assessment of quality of medical care). This is a review paper concerning performance and competence. Its main purpose was to look for definitions of the words "performance" and "competence", and to see if there was any empirical evidence for a relationship between them, using the definitions of performance ("what a doctor does in his day-to-day practice") and of competence ("what a doctor is capable of doing").

Chapter 6 (A method for introducing standardized patients into general practice consultations) describes in detail the results of the study of whether it is possible for a set of standardized patients to consult general practitioners repeatedly without being detected, in a health care system in which general practitioners have fixed lists of patients.

Chapter 7 (Assessment of the performance of general practitioners by the use of standardized patients) This chapter attempts to determine whether the actual performance of general practitioners, as assessed by standardized patients, meets predetermined standards for actual practice.

Chapter 8 (Does competence of physicians predict their performance?) A direct comparison between an examination setting and actual practice using undetected standardized patients.) contains the results of a study whose purpose was to determine whether competence (defined as "what a doctor is capable of doing") and performance (defined as "what a doctor does in his day-today practice" ) are related.

Chapter 9 (Conclusions and recommendations for research) rehearses and discusses the main conclusions of the work with standardized patients presented in this book and ends with some advice for further research with standardized patients.

\section{Appendices}

1. Detection form for physicians to register detected standardized patients

2. Instructions for the competence section of the study

3. The standards of care used

\section{References}

1. Tielens VCL, Mokkink $\mathbb{H}$, Mesker P. Het medisch diagnostisch handelen van de huisarts. Huisarts Wet 1985; 28: 44-8.

2. Vleuten CPM van der, Swanson DB. Assesment of clinical skills with standardized patients: state of the art. Teaching and Learning in Medicine 1990; 2: 58-76. 
3. Rosenhar DL. On being sane in insane places. Science 1973; 179:250-79.

4. Owen A. Winkler R. General practitioners and psychological problems: an evaluation using pseudopatients. Med J Austr 1974; 2:393-8.

5. Winkler RC. Research into mental heath practice using pseudopatients. Med J Aust; 2 : 399-403.

6. Amidi S, Solter S, Rashidian B, Zokajan A, Rasmjolan F. Antibiotic use and abuse among physicians in private practice in Shiran, Iran. Med Care 1975; 13: $341-5$.

7. Burr A, McCaughan K, Barrows H. The feasibility of using the simulated patient as a means to evaluate clinical competence in a community. Proc Annu Conf Res Med Educ 1976; 15: 295 .

8. Renaud M, Beauchemin J, Lalonde $C_{3}$ Poirier H, Berthiaume. Practice settings and prescribing profiles: the simulation of tension headache to general practitioners working in different practice settings in Montreal area. Am Publ Health 1980; 70: 1068-73.

9. Norman GR, Neufeld VR, Walsh A, Woodward CA, McConvey GA. Measuring physicians performances by using simulated patients. I Med Educ 1985; 60: 925-34.

10. MoClure CL, Gall EP, Meredith KE, Annel Gooden M, Boyer JT. Assessing clinical judgment with standardized patients. I Fam Pract 1985; 20: 457-64.

11. Anonymus. Show Biz (Editorial). JAMA 1973; 225.59.

12. Anonymus. Pseudopatients (Editorial). Br Med J 1974; $268: 729$.

13. McConaghy. Pseudopatients and evaluation of medical care Med J Aust 1974; 2: 385.

14. Anonymus. Simulated patients (Editorial). Br Med J 1974; 286: 399-400.

15. Anonymus. Pseudopatients and pseudoresearch (Editorial). JAMA 1975; 232: 59.

16. Rethans $J J$, Leeuwen w Y, Drop R, Sturmans F, Vleuten vd C. Performance and competence: two different constructs in the assessment of quality of medical care. Fam Pract 1990; 7: 168-74.

17. Senior IR. Towards the measurement of competence of medicine. Philadelphia: National Board of Medical Examiners 1976.

18. Lloyd JS. Definitions of competence in specialties of medicine. Chicago: American Board of Medical specialties 1979.

19. Norman GR, Tugwell P, Feightner JW. A comparison of resident performance on real and simulated patients. J Med Educ 1982; 57: 708-15.

20. Grol R. Kwaliteitsbewaking in de huisartsgeneeskunde. Krips Repro Meppel, 1986. Thesis. (Summary in english).

21. Lomas $\mathrm{J}$, Anderson GM, Domnick-Pierre K, et al. Do practice guidelines guide practice? N Engl J Med 1989; 321:1306-11.

22. Haynes $R$, Davis D, Mc Kibbon A, Tugwell P. A critical appraisal of the efficacy of continuing medical education. JAMA 1984;251:61-64.

23. Sibley JC, Sackeit DL, Neufeld V, Gerrard B, Rudnick KV, Fraser W. A randomized trial of continuing medical education. NEJM 1982; 306:511-5.

24. Reenders $K$. Hiemstra RJ, Bender W. Teaching fundoscopy to GP's in an OSCEsetting: failures and successes. In: Bender $W$ et al (eds). Teaching and assessing clinical competence. Groningen 1990, Boekwerk Publications: 374-8.

25. Barrows HS, Sirnulated (Standardized) patients and other human simulations. Health Sciences Consortium. Chapel Hill, North Carolina, 1987. 
Chapter 2: Simulated patients in general practice: a different look at the consultation

Reprinted from the British Medical Journal, 28 March 1987, 294, 809- 812

The paper has also been published as:

1. Rethans JJE, van Boven CPA. Simulatiepatienten in de huisartspraktijk: het consult anders bekeken. Ned Tijdsch Geneeskunde 1988; 132: 1162-6.

2. Rethans JJE, van Boven CPA. Simulatiepatienten in de huisartspraktijk: het consult anders bekeken. Consult van de huisarts 1988; 12: 34-37. 


\title{
Simulated patients in general practice: a different look at the consultation
}

\author{
J J ERETHANS, C P A VAN BOVEN
}

\section{Abstract}

To dewelop a better empirical basis for developing quality assessment in general practice three sinnulated patients made appointments willi 48 general protitioners during actual sturgery hours and collected facts about their performance. The simulated pattients were indistinguishable from real patients and presented a standardised story of a symptomatic urinary tract infection. Two month later the same general practitioners received a wriften simulation about a patient who had the same urinary tract infection and were asked how they would handle this in real puatice. Both results were scored against an existing consensus standard. The overall score for both methods did not whow any sibstantial differences. A more differentiated analysis, however, showed that general practitioners performed significantly better with simulated patients. It also showed that gencenal prictitioners answering the written simulation performed significantly more unnecessary and superfluous actions.

The results of this sudy show that the use of sinulated patients sems to show the efticient performance of general protothioners in practice.

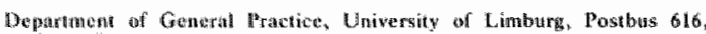

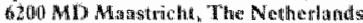

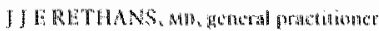

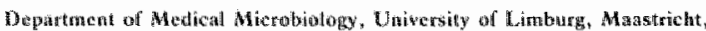
The Whetherlands

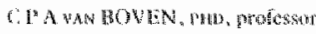

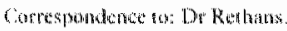

\section{Introduction}

In general practice as well as in hospitals effonts are being made th develop "ideal" standards and equitalen, logical, branchin flow charts for clinical performance. These developments am a improwing the performance of individual tasks, to promote a mon uniform performance by doctors, and to provide standards for the quality of care in solving clinical problems." "The use of suct standards has importan consequences-for example, a positive o negative evaluation of an individual doctor's performance and: reduced cost of heal th care for the communiry.

Most methods for developing standards are based on consensu: models, in which a group of general practitioners discusses the complant or muedical problem in antestion and decides on a snandart procedure for dealing with it. Srudies of the use of such standards. however, show wat doctors sem to do less well then such standards deem to be desirable ${ }^{n-1}$ General practitioners may not be acting as effriently as the designers of the standard would like, but the standand may also be too academic in that it does not allow for the circumstances acrually occurring ing general practice. It is therefore desirable to establish a standard of performanee for general practitioners based on the conditions of real lifie.

Methods of assessing actual performance may be either indirech, by seruding a questionnare to general practitioners to answer gacstions about problems with managing patients, or direct, by actuatly obserwing doctors while they are consulting. Comparisons have shown a discrepancy berween doctors" clinical performance as assessed by indirec methods and wat they acrually do when they are observed in their practice. 'Observed doctors omit actions that were expected from them from the resuls of the indirec methods. These studies, howewer, compared different groups of doctors dealing with different medical problems.

Though there is a substantial experience with the use of sumulated patients for educational purposes, ${ }^{4}$, and the simulated patient method has been described as the best method to assess the 
management of patents by doctors, few sudies have used sirruliated patents to research problems in actual practice. "3? This study aimed at comparing the performances of a group of general practioners when dealing wh a particular medical problem by an jindired method of assessment, using written detwils abou a simulated potient, and a direc method, using simulated patients in practice. We hypothesised that the general practitioners would do less in a consultation with the simulated patient than they woutd have indicated in their answers to the witten problem.

The swudy therefore had the following purposes: to investigate the possibility of asing trained simulated patients fo collect data atrout the realiry of the consultation; po measure the difference berween the pertormances of general practitioners when dealing with the simulated patient (what the doctor does) and the actions that the same general pracnitioners said that they would cake when dealing with a writen problem about a similar patient (what the doctor says be would do is and to measure the agreement between an alteady existing standard based on consensus model and the actual performance of general practitioners as shown by data gathered using simulated patients

\section{Subjects and nethods}

The medical problem in this study concerned a 30 yedr old woman who complaned abour painful micturition for one and a half days. This problem was constructed as a standardised role for three simulaned patients, whon were paid to participare in this project and had mo medical background. The problem was also written down in the form of a "simulation of initial medical problem solvinge "

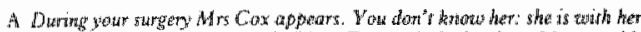

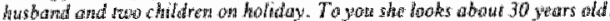
She compians af painf ut micturition for the past doy and a hall.

What would you like to ask her about her history?

H3 During your surgery hours Mis Smith appears. She is 30 wears old, and wot

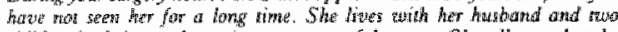
children in that in ow thause on a new part of the tonum. She tells you that she

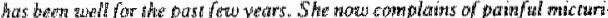

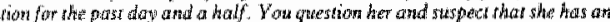

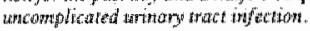

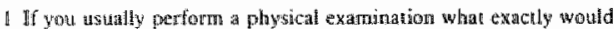
you do?

2 if you usually perform laboratory tests what exactly whonld you do?

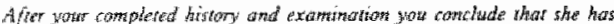

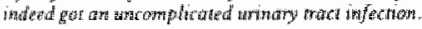

3 What would the your ronther trangagement (in terns of what you would porescribe and for how ing wilhat you woud tell her, and how you would arange a folow up)?

Whiven simulation of hitial ated dical problem solwing used in stady.

The wrimen form, meant to be used to assess medical competence, was a simplification of on modified essay question ( $\mathrm{E}$ de $\mathrm{Grad}$, unpublisher observations). The figure shows the format used in this study. The written format made it mecessary to meation the suspected urinary tract infection before the dochor cominted with the questuons on management idturing a real constifation the docror aceives answers from the patient). For this reason part A and part B were presented separately to the doctors (while reading part A they were tnable to see part $B$ ).

The facts collected about history, result of physical and laboratory examination, instrutions given to the parient, treatment, and followe isp were scored according to an existings stardatd on uringry ract infection developed at the University of Nimegen." The standard is divided into obligatory actions (considered to be necessary) and internediate actions (non essential but not harmful either). All other actions are considered to be shiperthuous (see ables I and II for the items. listed in the stand ard usedi),

The simulated patients were trained in their role as a patient and in

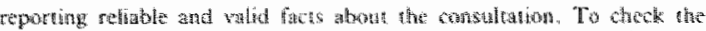

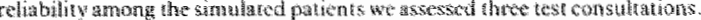

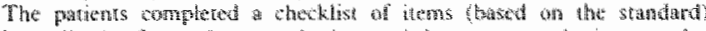

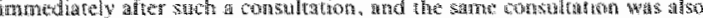

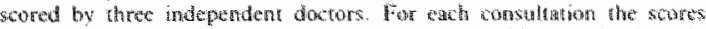

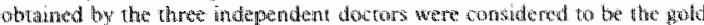

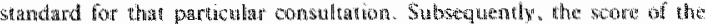

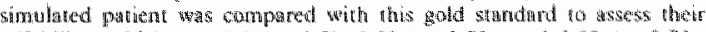

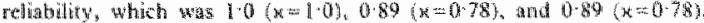

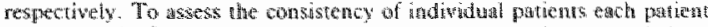
was retested ather sis weets wath the sanne constalnation frecorded on

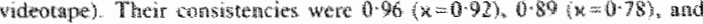

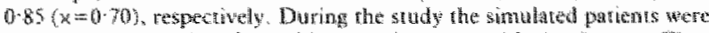

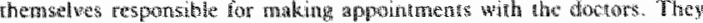
presented thenselwes as Mrs Cox (figure).

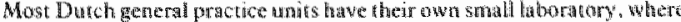

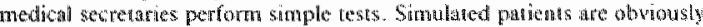
whatble fo undergo such tesss, se we asked the secretat ics of the particinstom doctors what kind of laborarory investigat fong a heir doctor would order them

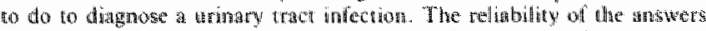

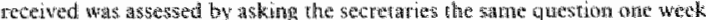
later. The agrement between their for and second answers was $99 \cdot 25 \%$

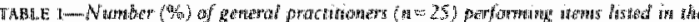

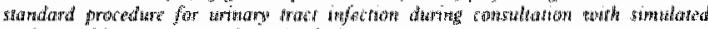

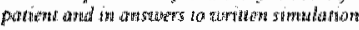

\begin{tabular}{|c|c|c|c|c|}
\hline & \multirow[b]{2}{*}{ 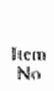 } & \multirow{2}{*}{ 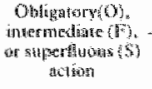 } & \multicolumn{2}{|c|}{ Profotmed } \\
\hline & & & 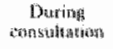 & $\begin{array}{c}\text { In stritgen } \\
\text { antone }\end{array}$ \\
\hline \multicolumn{5}{|l|}{ Hispory: } \\
\hline 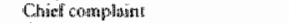 & 1 & (B) & $25(100)$ & $25(19 x)$ \\
\hline Fred cuency & 2 & 0 & $24(96)$ & $18(72)^{6}$ \\
\hline Orisell of compotatith & $x$ & 0 & 1302 & 11 [4] \\
\hline 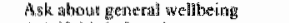 & औ & 0 & $\ln (x)$ & $11(4)$ \\
\hline Ask if this is first tinth & 3 & $F$ & 2201 & $22<88$ \\
\hline \multicolumn{5}{|l|}{ 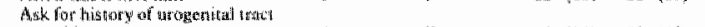 } \\
\hline problews & b & F & $\$\{16\}$ & $10 \quad(40)$ \\
\hline Vyaginal dischanger & $y$ & F & 520 & II (4A) \\
\hline Superftuous actionas & 8 & 3 & 520 & 12101 \\
\hline \multicolumn{5}{|l|}{ 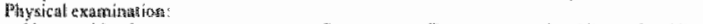 } \\
\hline 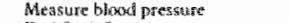 & 9 & r & I (4) & $1 n$ \\
\hline Foet fank for phan & 10 & $\mathrm{l}$ & $3112\}$ & $9(36)$ \\
\hline Superthous astivans & II & $s$ & 5003 & $13(52)^{*}$ \\
\hline \multicolumn{5}{|l|}{ Ingitguclions to pratient: } \\
\hline Expiajn diaignosis:y & 12 & 0 & 2.) 13 & $6(24)^{\infty}$ \\
\hline Exiglain prograsis & 13 & 0 & $12(48)$ & $2(8) *$ \\
\hline Ad vise woid ling afler intercoingse & $1: 1$ & $\mathbb{F}^{*}$ & $1 \quad(4)$ & $2(8)$ \\
\hline Advise drighting fhotre fluids & 15 & lim & U1 4 wath & $9(36)$ \\
\hline Discuss mutcrivion habils & 160 & $\mathrm{j}$ & $2(8)$ & $5(20)$ \\
\hline 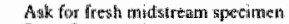 & 17 & 0 & (1) & \\
\hline Superfluious atcrioniti & 18 & $\mathrm{~S}$ & $3\{12\}$ & $2 \quad 4$ \\
\hline \multicolumn{5}{|l|}{ Tresument: } \\
\hline Anribiotics & 19 & 0 & $25(1040)$ & $23(9)$ \\
\hline Superfluous actions: & 30 & 5 & & \\
\hline \multicolumn{5}{|l|}{ Followit ap? } \\
\hline Selentiant & 21 & 0 & $20 \quad(6)$ & 190 \\
\hline Alerer ireatenem & ZI & 0 & $18 x+3$ & 16 (6) \\
\hline \multicolumn{5}{|l|}{ 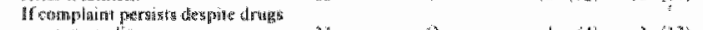 } \\
\hline 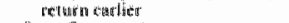 & 23 & 0 & & $3012 \mathrm{i}$ \\
\hline 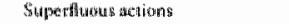 & $y$ & 5 & 1 (4) & \\
\hline
\end{tabular}

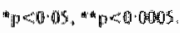

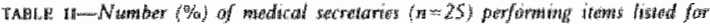

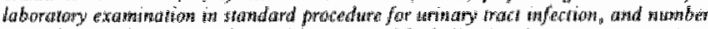

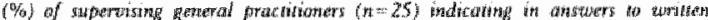

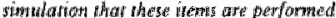

\begin{tabular}{|c|c|c|c|c|}
\hline & \multirow[b]{2}{*}{$\begin{array}{l}\text { Whenri } \\
\text { Na }\end{array}$} & \multirow{2}{*}{ 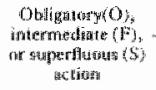 } & \multicolumn{2}{|c|}{ Performed } \\
\hline & & & $\begin{array}{c}\text { Hy } \\
\text { oforetary }\end{array}$ & 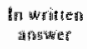 \\
\hline 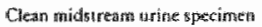 & 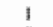 & 0 & $1(4)$ & $2<B$ \\
\hline 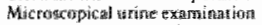 & 2 & 0 & $2 H(B A\}$ & $200 \mathrm{~s}+40$ \\
\hline \multicolumn{5}{|l|}{ 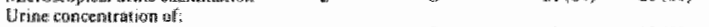 } \\
\hline Glingose & $y$ & 0 & 2464 & 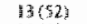 \\
\hline Notrite & 4 & $\mathrm{H}$ & 9096 & 10040 \\
\hline Allowingin & $s$ & $\mathrm{~F}$ & $23(9)$ & $11069^{\circ}$ \\
\hline 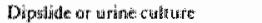 & 6 & $\mathrm{~F}$ & 14 & \\
\hline Supperfluous actions & $\%$ & 3 & 14 & \\
\hline
\end{tabular}

pisencos. 


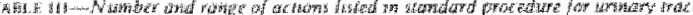

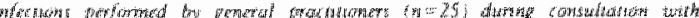

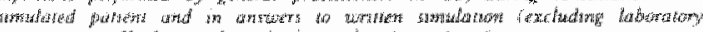

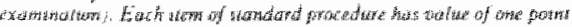

\begin{tabular}{|c|c|c|c|}
\hline Matiars: & 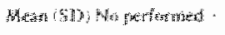 & xhes & xy Of of \\
\hline \multicolumn{4}{|l|}{ Hous: } \\
\hline 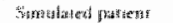 & 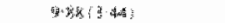 & $41 \%$ & \\
\hline 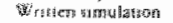 & 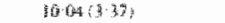 & $4-18$ & \\
\hline \multicolumn{4}{|l|}{ 1. } \\
\hline 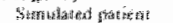 & 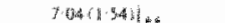 & 4,5 & 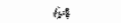 \\
\hline 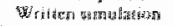 & 9. $2411804 \frac{3}{3}$ & $2 \%$ & $4 i$ \\
\hline \multicolumn{4}{|l|}{ Wherpediate. } \\
\hline 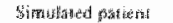 & I. & 0.3 & 24 \\
\hline 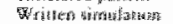 & $\left.786\left[1 \cdot 3 x_{3}\right]\right\}$ & 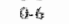 & 페서 \\
\hline \multicolumn{4}{|l|}{ 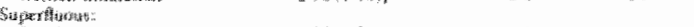 } \\
\hline 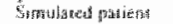 & 9) & 6.8 & \\
\hline 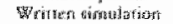 & 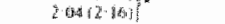 & 0.8 & \\
\hline
\end{tabular}

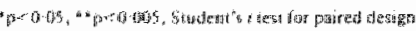

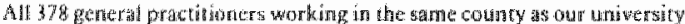

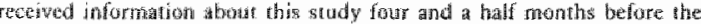

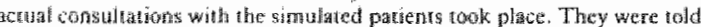

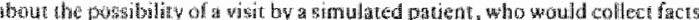

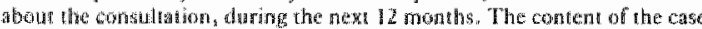

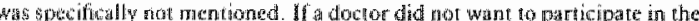

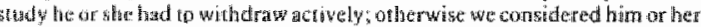
(n) be a pusshe participant.

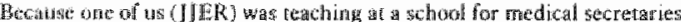

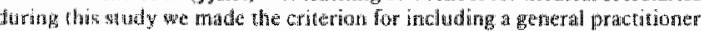

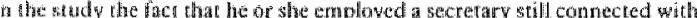

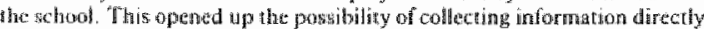

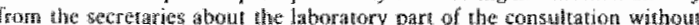

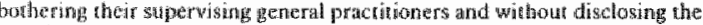
greturil diasign of the study

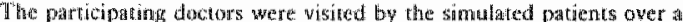
period of four and ta half weeks. Two months after the wisits all doctors were

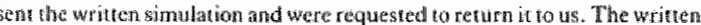

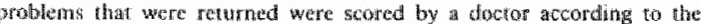

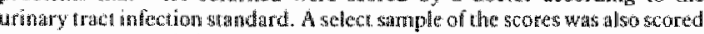

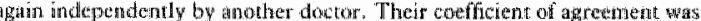
$019(x=99)$.

The wo tahed McNemar tem for paifed dats was used to compare the propartion of general practioners performing atr action during arasulation with a simulated putieg and the proportion of genera

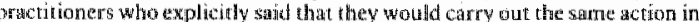

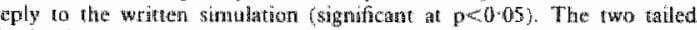
Siudent's 1 tesl for paired data was used to conpare the ional number of

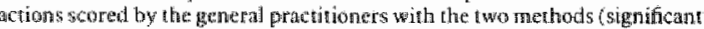
ail $p+0,050$.

\section{Acsultis}

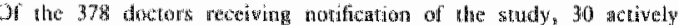

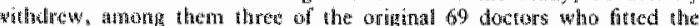

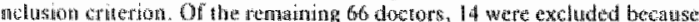

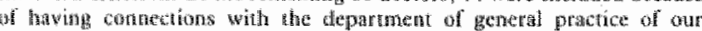

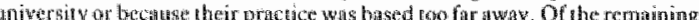

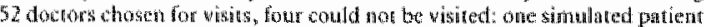

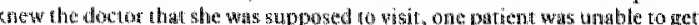

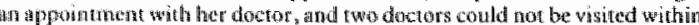

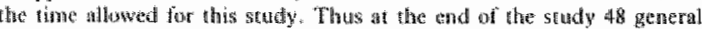

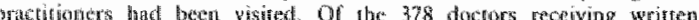
singutution, 19 (57\%) raturned it, inctuding $27(56 \%)$ of the 48 docrors who

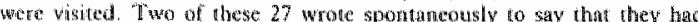

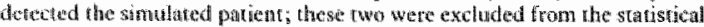
untalyesing

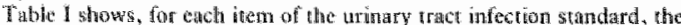
proportian of general practitioners who acrually performed the acrion as

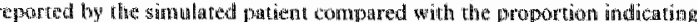

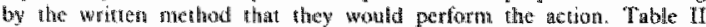

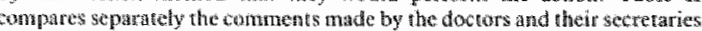

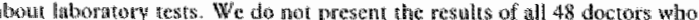

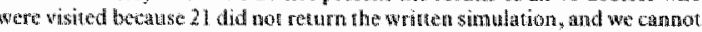
therwore meke anv comparison. The proportwon of downes per item of these

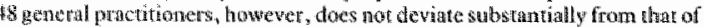
the 25 whents teported on there.

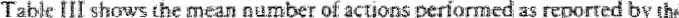

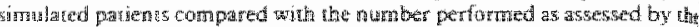

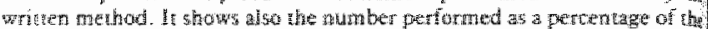
number uranomended by the siandard.

\section{Discussion}

This study shows that sinulated patients can produce reliable and walid data about the periormance of doctors in actual practice. Only rwo of the 48 general practitioners who were wisited paported afferwards that they derecied the simulated patient. We have not reason to suspect that others also did, though we admit that we canmor be sure. Some doctors, on receiving the writen simulation, may have recognised the visil of the simulated patuent becanse they wrere able wo rall the fearures of the case. To prevent the doctor from recalling the consultarion with the simulated patient we introduced Mrs Smith in the written method. It could be argued that the section on management in the writken simulation no longet applies to the visit of the simulated patient Mrs Cox. This is true, but there is no strict medicall reason for the management of Mrs Smin and Mrs Cox lo differ in the irems recorded here.

Table $\mathbb{I}$ shows clearly that in taking the patient's hirory, giving the parient insiructions, prescribing treatment, and following up the doctors performed more obligatory actions during the visit by the simulated patient than indicated by their corresponding intentions in the writem simulation (except for item 23). The total score for both methods, however (table III), shows that more acts were intended to be performed than were performed in real life, but the difference was minimall and not significant. A more differentiated analysis, however, silhows that for the obligatory aicts doctors do more in reality than they say that they intend to do as measured by the written simulation, a finding that is at variance with our initial hyporthesis. For the intermediate and superfuous actions, however" the findings were inst the opposite.

These results are surprising. Most surprising is the finding that there seems to be a clear distinction berween obligatory actions on the one hand and intermediate and superfluous actions on the other. We think that perhaps during the consultation with the simulaced pacient, under the pressure of time, the docrors deal effoiently with a routine problem. During the writren sumulation, however, they tend to want to show their knowledge, exhibiting an examination techmique and forgeting about nomal rowtine procedures. The actions and inventions for laboratory examinations show fewer differenes, but this is the only category in which doctors are not being compared with themselves. Such results give reason to bebieve that using simulated patients shows a pattern of efficunt performance (for the most essential issues) by general practitioners.

We are well aware that content specificicy could affect the results. "More research is therefore necessary to investigate whether the simulated patient method also shows this special partern of effrcien performance when more doctors are dealing with other medical problems. Practical problems, such as dewising different ways to enter practices and deciding on which medical problems can ba used in in simulation, still have to be resolwed.

This study shows that the use of simulated patients in practice resuls in more (and probably more relevano) information about the performance of general practitoners than other existing methods. This is shown most clearly by the category "instructions to parients." "The answers obtained by the indirect method would lead to the conclusion that doctors forget to tell their patients about the diagnosis, while the direct method shows that doctors are giving excellent instructions.

During consultation with a simulated patient the participatimg doctors performed only $64 \%$ and $24 \%$ of the obligatory and in ermediate actions, respectively (table III). We have no reason to suspect the participating dochors of having low standards of care. We therefore think that these results indicate that the consensus standand does not take into account sufficiently the reality of pracuce for general practitioners. "The standard should have a better empirical basis. The simulated patient method cotsld be the method of providing this basis. Siandards should almost certainly be 
evolved in a praterical setcing even if deweloped initially in an abstract manner by discussion groups. In any case, the measurement of gerformance against standardis should be carried out practically and not by natu abstract questionnaire.

The results of this study encourage the wase of the simulated patient method as a basis for establishing the actual performance panterns of general practivioners. The full documentition of these patents in daily practice still seems to be areat challenge.

We thank D L Crombie, C die Gens, J Hendrix, P Hobus, A Knatnenis,

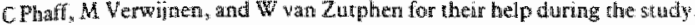

\section{References}

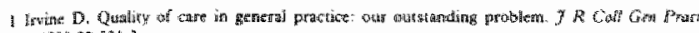

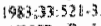

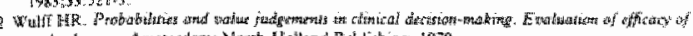

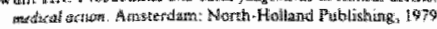

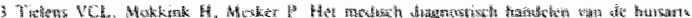

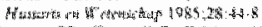

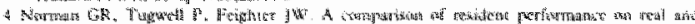

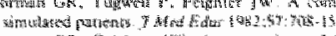

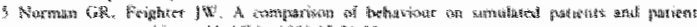

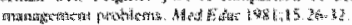

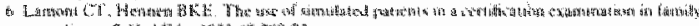

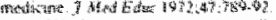

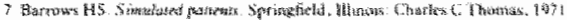

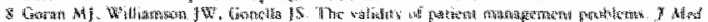

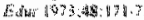

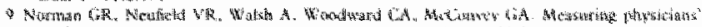

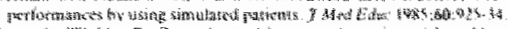

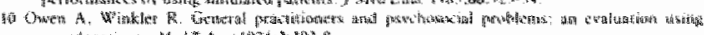

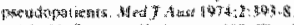

11 beriry

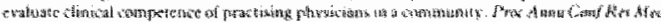

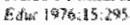

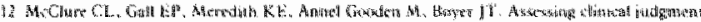

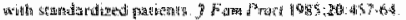

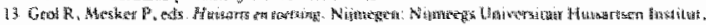
$19 \mathrm{~g}$

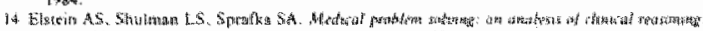

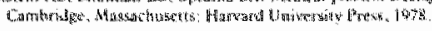

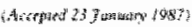




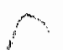


Chapter 3: De dokter onderzocht: simulatiepatienten met mictieklachten op het spreekuur wan de huisarts (The doctor examined. Simulated patients presenting micturition complaints in office encounters)

Reprinted from Huisarts en Wetenschap, 1988, 31, 3-6 



\section{Summary}

A simulated patient is a person who has rehearsed a role as a patient, which role he then plays in an encounter with a doctor. Initially simulated patients were used only during medical vocational training in (self) evaluation of trainees; later, they were also used in real practice situations. 7-9 In an effort to establish the feasibility and effectiveness of this method for Dutch general practices, a study was carried out of the performance of general practitioners confronted with the complaint 'painful micturition'. The casuistics chosen concerned a woman aged about 30 who had for the first time developed a symptomatic urinary tract infection during a holiday and presented to an unfamiliar general practitioner with the complaint 'painful micturition'.

For reporting the data on the encounter the protocol 'Micturition complaints' of the Nijmegen University department of General Practice was used. ${ }^{16}$ All items relevant to the casuistics chosen were culled from this protocol, and this list of items was used as measuring instrument for the simulated encounter.

The study was limited to 69 general practitioners who had a traineepractice aide completing a period of practical training in their practice. Via the school for practice aides, information was obtained on the laboratory tests used by these general practitioners when confronted with micturition complaints.

From a pool of some 100 simulated patients three women aged about 30 were selected who were considered able to give a detailed account of a simulated encounter afterwards. These women were carefully trained to play their role and to report reliably on an encounter.

The simulated patients were given samples of urine to take to the encounter. It was ascertained that on the day of the encounter all had similar, unmistakably infected urine samples with them, divided into two portions. If required, the woman could give the general practitioner one portion; she could give the other portion if she was asked to produce urine "on the spot'. A seventh, identical urine sample was tested at the university microbiological laboratory for nitrite, glucose, albumin, $\mathrm{pH}$, leucocytes and sediment (more or less at the time of the women's encounter in general practice). An urinary culture was started as well.

Of the initial population of 69 general practitioners, 23 dropped out for various reasons. Of the 46 remaining general practitioners, 78 percent posed only three anamnestic questions and only one asked for a washed midstream sample. Other striking findings were:

- 35 percent of the general practitioners used nitrofurantoin as therapeutic agent of choise;

- only 19 percent of the general practitioners prescribed an antimicrobial course of less than seven days; 
- of the general practitioners who made an appointment for a follow-up, 92 percent asked the patient to report back 5-10 days after the start of the medication;

- 40 percent of the 'mandatory' items were omitted;

- active general practitioners were actice in all categories; those who did little, did little in all categories.

According to the guidelines of the protocol used, an item was characterized as mandatory if it involved a truly necessary activity; its omission was regarded as negligence. In this study this would imply that the general practitioners were negligent with regard to 40 percent of the mandatory items. The study population consisted of general practitioners who met the criteria formulated by the schools for practice aides. Consequently there is no reason to assume that especially these general practitioners would provide poor medical care; it seems more probable that the Nijmegen protocol, despite exhaustive validation, accounts insufficiently for the reality of an encounter; in other words: the standard set is too high. The findings raise questions, both regarding the way in which standards are set and regarding the extended training of general practitioners. 


\title{
De dokter onderzocht
}

\author{
Simulatiepatient en met micheklachen op het sprekuw wan de huisarts
}

\author{
JAN IOOST RETHANS EN CEES VAN BOVEN
}

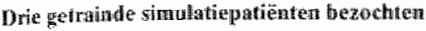

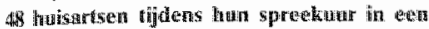
peblindeerde setting. Zij presenteerden zirle als gratienten met en symptomatiche aripus: wegunfecte en registreerden wath de linutsartsen rijuens het consult wogen an dedem.

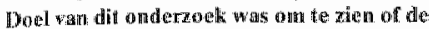
simulaticpatient-methode hambar whe

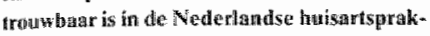

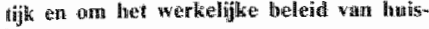

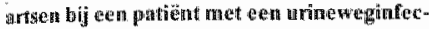
Life vant te stellen. De resullater werden gescowd mett behulfis van het protocol 'Mictickbchten" wan het Nijmedgs Universibair Haisartion Instituut. De sinulatiepatienten

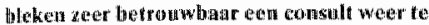
fumnen geven en bifechts wee wan da 48 huisartsen ontmakgerden de sinumatiepa

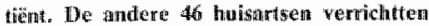
slechts 60 procent wan da handelingert die valgens hed protocoll "obligatat" wiren:
\end{abstract}

\section{Inleiding}

Protocollaire geneeskunde en intercollegilat toetsing zijn actuele on witkelingen in de huisantsgeneeskunde die v1:gen om methoden die de werkelijkheid van het spreekur zichtbaar ma ken. ${ }^{2}$ In de wisie van het Meachands Huistarsen Genoorschap dienen do in

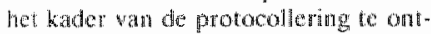
wikkelen nomm an te shiten bij de hadige gang varaken in de praktigk en fevens weanschappelijk verantwoord ace zijn. ${ }^{3}$ Ook De Han en Hollenbeek Brouker komen tot de conclusie dat theoretische normen pas werkelipk betrouswabar zullen zign, als zij bin de prak. tijk worden geijkt: "De normen wan het medisch bandelen dienen fe worden ondeend an he veld wan pratherende muisartsen' "Dis Houd in dat het noodzakelik is om betrouwbare informatie bver the handeten in de praktijksituatie te verkriggen

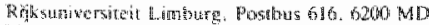
Hawrenthe

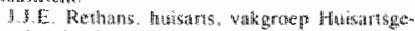

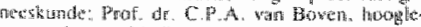

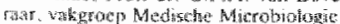

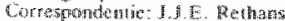

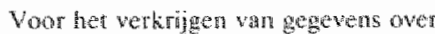
het handelen wan hunsartsen tijdens bet dagelings spreekur zijn versohitlonde methoden ontwikkeld:

- enquêrte;

- andyse wan het kasatsystem (char auditi);

- papierempatientenproblemen

- contact mer een simulatiopatient in een laboratoriumsituatie, eventueel op video vastelegd:

- Gbservatie van het spreckur door aen obsenvator, eventued gecombineerd met een andio-opname:

contact met ocn sirmulatiepatient tijdens het spreekuur, wastouj de sinu. latiepation gegevens over het consult verzantelt.

In welke mate deze methoden de werkeligkheid wan het spreskurcontact werergeven, is overigens walk nite dudelijk Zo werd eet discrepantie gevonden tussen de gegevens die via observatie waren verkregen, en de rapportage door de husartsen zell.

Eer simulatiepatent - de aan en methodiak zijn bedacht door Barrows $s^{6}$ - is iemand die een rol als patient heeft ingestudeerd en deze rol vervolgens speelt in een contact met een arts. Soms worden echte patienten gebruikt, die dan hun eigen ziekte in een gestandaardiserde rol presenteren. Aanwnkelijh werdar simulatiopatenten alleen iijders de medische opleiding gebruiku ter (zell)evaluatic van studenten. later ook in echte praktiksingaties.

Het toe passen van woor de arts ontokende en niet herkende simulatiepatibnter in de dageligkse priktijk met hot doel informatie over zijn handelen to verthiggen heeft seberpe krivek onder vonden, mat marme omdat lie onethish zou zinn artsen zonder hum robdewaten te observeren. " Een probtecm is onk de betroumbartheid wan de rappontage vat de sirrulatiepatient over een con5.16. ${ }^{12} 13$ Recent is echter abngetoond dat systematische oelening vall simulatiepatienten to en anuardbare ni-

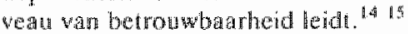

Oim de loepasbarheid en bruikbar heid van deze methode woor de Neder- harde husanspaktijk vast to stellon.

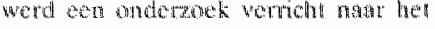
hardeten val hitsaren bij da kiach piju bij her plassen"

\section{Methoden}

\section{Micheklachten}

De gekowen easurbek hat betrokking

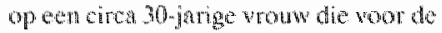
eerste keer ean symptomatische urink-

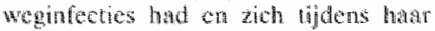
wakane meldde bij een hat vreende

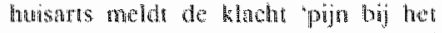
plassen. Dit he instuderen van de rol werd, betwate am her conted natar woforn brengen van de kach veel awn

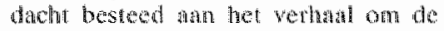
klacht heen frakantie. vertekerings vom, beroep, ect.

De gegevens die door the simulatiepa tient over het consult zouder worten gerapporterd, mosien uiterard eenduidig worden wastgetegd. Hiervoor is gebruik gemaakt wan het protocol 'Mictiek achten van hen Nijnecgs Universi tair Huisartsen Institum (NUHI). ${ }^{\text {to }}$ Voor dit protocol is gekozen. omdat de

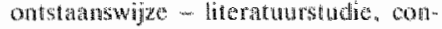
sensus-methode en withester ho de pratltjk - een garannie leck woor een goede aamsliting op de praktijk. Uit het pronocol werden alle items gedestalleerd dic og de gekozen astistick betrekking hadden (ubet I) deze itenlijst is getbrukt als metimstrument woor het smulatiecontact.

\section{Huisismtsen}

Alle 378 huisartsen in de regio van de Rijksuniwersiteit Limburg kregen ongeveer vier manden voor het onderzock een brief waran vidleg over de varastelling werd gegeven en wanin werd

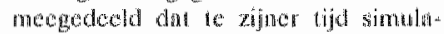
thepatibnter aen antal prakijiken zoader bezocken en zouden regigraren wat

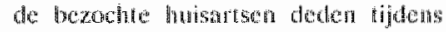
hat consult. Nadrukkelik wort niet ver meld om wolke klacht het ging. Huisartsen die bewwar togen the onderageh hadder, konder wich afmelden; bij geten berich werd atugenomen dat mentr toestamming verlesende.

Ondat het besend bif michekiachuten wordl kenment door een groot antrital handelingen dat zich in het laborato.

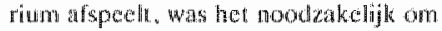
ook over dis onde wedes gorede informatic te werlorigen. Denarom is hel onder" zoek in cerste armeg beperkt fol de 69 huisantsen dic en staghate van een doktersassistentertopletiding in liven prakijk hadden. Via de school fen met toestemming van de opledding werd 
informate ingewonnen owe de lisboraworamianostich bit molicklachen

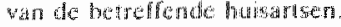

Hutsartsen die mowilik a beretken waren. en haisansen die op de hoggte wouden kurnon zigh wan do te presente.

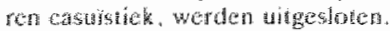

\section{Sintallatiequatienten}

De Rijksunversinen Limbarg besclakt via hatar afdeling skillsh buer cen pool

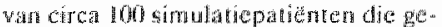
haruld worden ten behoeve wan her medisch onderwifs. Uit deze groep werden Grice yrowwer wan rond de 30 far gekozen, die in shat werden geach om een simulatiecondact na alloop goed weer we gever w, en bowendicn gredurende zes wethen vior ochtenden per weak beschik" bast wath. Deze dric vrouwen werden wervolgens op het skillswly getraind in hed spelen van hum rol on het betrouw-

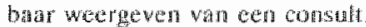

Voor de evaluabe hiervan waten tijdens to trainingen drie meetpunten wagestedd, met tussenpozen van drie we. ken. Tijdens de matingen wond op the Skillab cen video-opname plats valn een spreekumeonthet van een huistats met alle dre de simulariepatienter. Dexa video-ponnen werden onathanklijk van elkais" beoordecld doon drie arisen atan do thand van de genoende iremlist. Adus werd een qouden stan

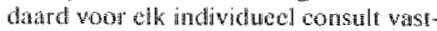
gesteld. Anders gezegd, de antwoorden dite de beoordeladars op de scoringsijst inwaldan, golden als de 100 procentscore van dat consult. De behaade scores van deze interbetrou watarheidsmetingen waren respectievelijk $\mathbb{1}, 0$ (kappa $=1.0 \% .0 .89(\mathrm{kappa}=0.78)$ en 0.89 $($ kappdi $=0.78)$.

Nats the interbetroumbaarheids meting werk ook de infrabetrovwbarHeid van do simulntiepatienten gemoter don iefer whin hen hum elgen derde wheting. dibs op video was opgonomen. nat vier waken opnienw te laten scoren. bo patichen onvingen extra traming op de vragen die zij onjust invulden. De

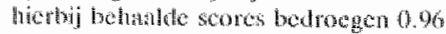
$(\mathrm{kappa}=0.92), 0.80(\mathrm{kappa}=0.78) \mathrm{ch}$ (0.4.5 (kappa $=0.75)$.

\section{Urine}

1n trun rol als partion hadden de simulathiphtichaten urinemorsters bij zich. Er wert woor getorgh dat alle vouwen per onderzoekdag dazellde, dudelijk gen. focteende urine bij zich haddan on wel vereceld over rwee portics. Enn portie kon de whaw desgewaged overhandi-

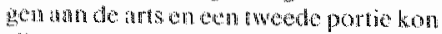
zij owerhandigen als hat werd gewrataged on ter plekthe urine te producesen. Zij gool dar op de $\mathrm{WC}$ de weele pottic over in het annorem the thentie.

Een zevendle identek monster wan

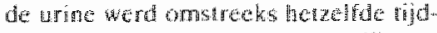

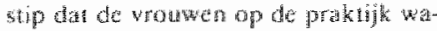
ren op hei michohologiscl Maborato rium van de uniwersteit bekeken op

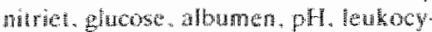
ten en stiment. Teverns wherd een uncult ingezer. Aldus werd per dag een gouden urinestandard asergesteld.

De berouwbatheid wan de verstrek te geverens door de bokrergascistentes wert gemeren door her de informatic over het laboratorium twe what is vra. gen, warbi de assistentes de cerste keer niet wister thet dit nogrnats gevrangd zou worden. De owereenkomst ussen hun gegevens van de cerste on
Wwede keer whace wa 99.25 procen

\section{Scones}

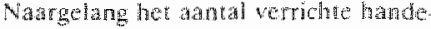
lingen hebben the de arsen scores begekend woor hun consuhen. Per werrinh item werd l pun toegekend. Am do

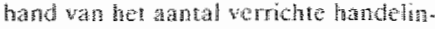
gen konden we nagatu hoe de groep huisantsen scoorde op hei gebruike protocol, zowel woor het totad ats voor de crtegoriect obligat en facultater. Ondat de categorie overbodige handelingen in principe oneindig groot is. koo hier gex n percentage over worden benekend.

Om thezien of thet mogelik was de betrokken huisartsen te beonrdelen op hun werk wize, hebben we ook gennalyseerd in hoeverre er samenhang is tus-

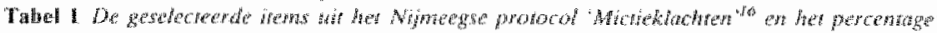
Whatren (n)

\section{Harmente}

1. Aard tan de kacklth

2. Frequentie var de micitie

3. Hoelang hestaat de klacht

4. Algermeen welbevinden

5. Recidieweri nagaan

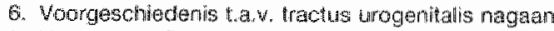

7. Vraagt naar fluor waginalis/pruthus wulwate

8. Overbodige harudetingen aramanese

0

0

0

0

E

F

E

ON

Lichamelijk onderzoek

9. Tensiecontrole

10. Slagpininierloge-onderzorle

1. Overbodige handelingen onderzoek

Eigen laboratorium

12. Gewassen middenplas, andefzow max. 2 uur na lozing

13. Sediment

14. Urine gliucose

15. Urime nitritet

16. Dipslide of urinekweelk

17. Urine albumen

18. Overbodige handentingter baboratorum

\section{Voorlichting}

19. Uitleg diagnosa

20. Prochose besprekery

21. Advias goed witplassen na coitus

22. Vael ditrkent abiseren

23. lngar op mictiegewoonten: hygüne

24. Vorse gewassen unine wagen

25. Owerbodige handelinger vortichting

\section{Madicatie}

26. Antibioticina richatle

27. Owerbodige handeanger madicatie

0

ov

\section{Torucbestellon}

23. Controle atspieken

29. Controle trine-ondaragk na afloop van de kuur

30. Aangeven dat de patient bil armbouden wark de klacht concianks medicatiol eerder harug moet homem 3\%. Owerbodige handelingen controle

100
89
46
50
78
9
17
20

0

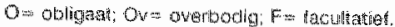


sen respectevelijk het adatal obligate. facultatieve en overbodige handelingen en tot foral verriche aasual handelingen.

\section{Resultaten}

Van die anvankeligke populatie van 69 huisarisen 7 inn er 23 afgevallen:

- 3huisartsen meldden dich na do anm kondigingsbrief af;

- 4 huisartsen werden geschrapt in werband met mogelijke bekendheid met do te presenteren klachen;

- 10 huisartsen werden niet bezocht in verband mer de afstand;

- I husisars weigerde de patient te ontvangen:

- 3. huisartsen werden nien bezoch in werband net tijdgebrek.

- 2 war de 48 bezochte huisartsen meld. den achteraf da ze er bijna zeker van waren dal ze de simudatiepatient hadden ontdekt.

Di resultaten wan de consulten wan de restertende 46 huisartsen zijn vermeld in rabed 1 . Mentit thy onder meer dat 78 procent van the husarisen slechts dre antmestische vagen stelde, on dat slechts een huisarts on gewrasen middenplas-umine voeg.

Wit tabel 2 dink dat 40 procent vande "oblgate" items niet is verrich

In de abollen 3.5 zijn eakele tems van het protocol gedetaillerder whigewerkt:

- nitrofurantome was voon 35 procen wan de husarisen een eersteneus prepartat (kobel 3):

- slechrs 19 procent van de muisartsen schreef een antionicrobiele kur voor vanninder dan zeven dagen (rabel 4 ):

- van de ludisansen die een nacontrole afspraken, mante 92 procent die alsprazk voor $\$$ tot 10 dagen na het begin van de medication fobel $5 \%$.

Whit net obliykt tenslotte dat artsen die in cotal vee: handelingen verricturen, in allie categoriean veel dedan, en dat artsen die weinig deden, in allos weinig deden.

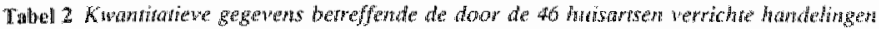

\begin{tabular}{|c|c|c|c|c|}
\hline Haneolingery & Quemiddalde & Sproiding & $\begin{array}{l}\text { Wo van date } \\
\text { standalard }\end{array}$ & $0.25-0.75$ \\
\hline Totand & 13,2 & $7-23$ & & $10,7-15,2$ \\
\hline Obligaat & 8,41 & $4-12$ & 60 & $7,0-10,0$ \\
\hline Fact llatief & 4,04 & $1-9$ & 36 & $3,0-5,0$ \\
\hline Owerbodig & 0,76 & $0-8$ & & $0,0-1,0$ \\
\hline
\end{tabular}

Tabel 3 De voongeschrewen madicatie aph het

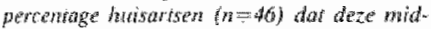

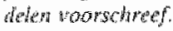

\begin{tabular}{|c|c|}
\hline Medicatio & Percentagle \\
\hline Milroturantoine & 35 \\
\hline Cotrimoxazol & 24 \\
\hline Sulmamethioli & 15 \\
\hline Triptetoptirn & 9 \\
\hline Plpemidinezkntext & 9 \\
\hline Noroxint & 7 \\
\hline Armoxicilling & 2 \\
\hline
\end{tabular}

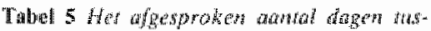

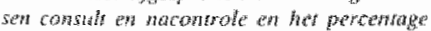

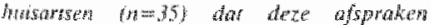
moative.
Thbel 4 De dikter wast de worgeschreves kwater en het percenfage hisartsen $(n=46)$ dar dere kuren woover hreef.

\begin{tabular}{lr}
\hline Arntal dagen & Percentage \\
\hline 4 & 2 \\
5 & 15 \\
6 & 2 \\
7 & 48 \\
8 & 2 \\
10 & 35 \\
\hline
\end{tabular}

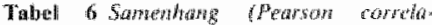

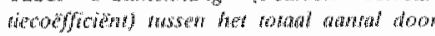

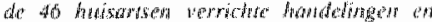

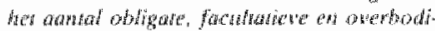

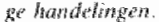

\begin{tabular}{lcc}
\hline Handilingen & Tolat & $p$ \\
\hline Obiligat & .74 & 0.000 \\
Facultates & .77 & 0.000 \\
Overooding & .72 & 0.000 \\
\hline
\end{tabular}

\section{Beschowwing}

Niel terder is op deze maner informatio herkegen over het forelijh bandolen

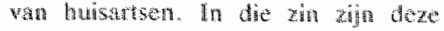
pesultaten don ool wiok

Volgens de richthonen van thet gebruikte protocol is een hindeling als obligatl gekentherkt ats het om cen edht noodzakeligke handeling gate; het weglaten ervan word beschout als matatig handelert. In dit onderzoek tou dat be-

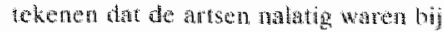
40 procen wate doblighte brems.

De onderzoche groep bestaxt wir husartsen die voldoen ata de miteria die opleidingen voor doktersassisacules stellen. Wij hobben dan ook geen reden om an te remen dat speciald wo huisurtsen sleche medische zorg zonto den vertenen. Hen lijk ons warselningiijket dat het Ninmeense protocol, ondanks de uthoerige wijze wan validoring, toch te weing rokening houd me? do werkelijkheid wan een consult, dat wil zeggen dat de thotm to hoog is gesteld. In de literaturir ower do diagnos-

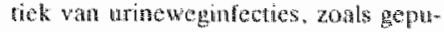
biceerd in Masarts en Werensehop inde lantse 15 jar. kon bijoorbed ka op keer de gewassen midtenplas-urine, onderzoctu met de dipslide-methode als enige juiste nethode trit de bus. Iir ons onderzock heeft slentus fen van do 46 hailsorisen hiervan gebruik gemilak!. Dit rocpt vratgtckens op ten anmien. van de maner wathop nomen worden gesteld, en ten anzien van de nawcho. ling die huisentsen ontwengen.

Via getrande simulaticpatiénten feiro. lijke gegevens over het handelen wan hübartsen verzanclen, is om verschillende redenen aanuek kelijk an zinvol.

Allereerst zal het moetijic zoing een mothode wa datwerranding in do

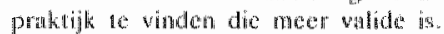
Just het fon dat do hasarts an kuestic niel weet dat or lijdens cen consult gegevans over zijin landelen wosten verzamold. makt a da hij de kars thijg!

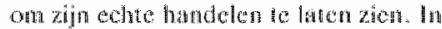
rweede fase wan dir onderack bijwor.-

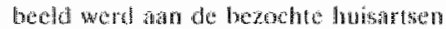
gevatitgd wat ze in de" dongelijkse prak-

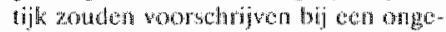

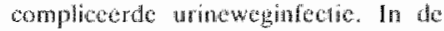
schrificlijke antwooten werd doon 35 procent vath de hussintser? sulfamethisol als earse keus werd angegeven; 26 prowent koos woos corrimoxizol an 17

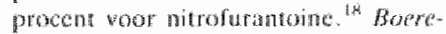
mo wond zells when enculate onder

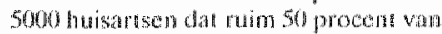
de Mederiandse huisarsen zou kiezen 


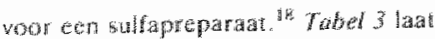
echer zien dat as samenselling wan de

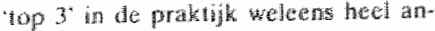

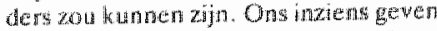

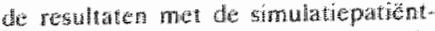
methode adr dat on zijo minst voordettighed to gebouen met hed treken wan

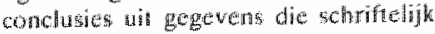

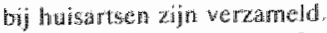

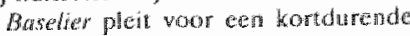

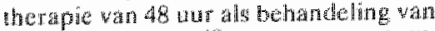

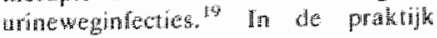

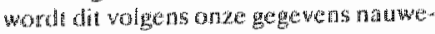
lijks gedan.

Een ander voonded van het getorul van simalatiepatenten op hel sprestu

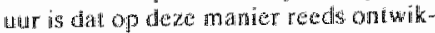
kelde protocoller woor hes huigarisgc. neeskindig handelen getoetst kunnem worten on hut hatbacheid an dat

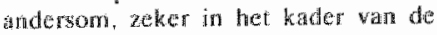
meeriarige beroepsoplading, wh hus armengetoetst kumen worden op hun handelan. Door het achter far huis. artsers vatgen watrom man whel of nit een hadeling verrichter, kan tevans

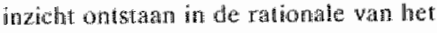
dugefijks hrindalerie.

Bij aamvang van dit onderowek heblen cile simulatepatignten onder grote psychische duk gestatan, wootal door de gevolgde manier van toestemming vragen ande lussarisen. De simalatiepay-

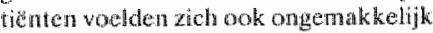
toen zij merkten dat de ansen slachin scoorden" op de itemlist, terwijl zij het gevoel hadden adequat ke worden bekandeld. In de loop van het onderzoek werden de patienten steeds meer ontspanten en tenslotte waren zäl zonder meer bereid bij aen volgend onderzot weer mee te doen.

Wij realiseren ous lerdege dat nog zeor weel problemen bil het invoeren wan dete simalatepthent-methode rige ten worden overwonnen. Mer name ale praktische witwoerbarnheid an de uthische problematick vagen nog om

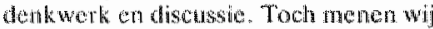
dat deze methode, noits verantword en goed gebrukl, een goed nzich kan werschatifen in de werkelijklneid wen de spreckkamen wan te husars.

\section{Dank luetuiging}

Wij withen hiegbij onde dank aispreken an C. de Oeus J Hendrix, P. Hobus, Y. Kanser,

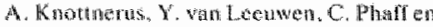
W. van Zurghen voor hun bijdrage an deze shutio.

\footnotetext{
"Wan den Dool CWA. Pro protocol. Huis arts Wel 1984: 27Guppl 8h: 379.
}

7 Wigersma L. Doetsellingen en begerkin-

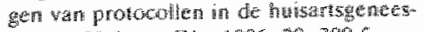
Kunde. Huscasts Wed 1.986: 20:3005.

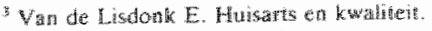
Prisarts We 198 : 24: $250-1$.

A De Hosn M. Hollerbelk Brouwer H. Normien woor het wokteren vall de hutistris Men Contac 198\%: $37: 1650.3$.

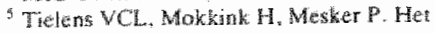
medisch diathowstisch handelen was de huigart. Hustate

6. Eurri A. McCaughan K. Barrows H. The feratility of asing the shmulated patient as a means to evahuate conical competence of pracuicing plysticians irs as commurity. Rime-confercincen 6,2 p. 295.

Barrows HS Simulated patients. Spring-

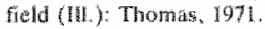

Sowen A. Winker R. General practilofues: and psychoscocial problems: an evaluation bsing prevdopatients. Med J Austra lis. $197.4 ; 2: 393-8$.

"Rosemhan DL. On being sane in insane places. Science $1973,17 \%: 250 \%$

10 MrConaghy N. Psendopatiemts and evaldiation of medieal practice. Med I Aust 1974: $2: 385$

it Anonymous. Pseudoparicnts and pseudoresench [Editorial]. JAMA 1975; 232: 59\%co.

12 Larront CT, Hennen BKE. The esse of simulated pathents in a crevification examinanson is candily medicine. I Med Edac 972: 72: 789-95

1) Norman GR, Tygwell PB, Feighter $\mathbb{J W}$. A comparison of residen performance on rent and simulated pattonts. I Med Edduc $1.982: 57: 708 \cdot 15$.

* McClure CL Gall EP, Meredith KE. Antw nel Gooden M, Boyer JT. Assessing clinial qudgment with standardized patients. I Fan Pract 1085; 20:457-64.

1 Norman GR Neufeld WR, Walsh A. Woodward CA, MoConvey CA. Measu* ring physicisans" performances by using simutated patients. J Med Educ $1985 ; 60$ : $925-34$

to Grol R. Mesker P. red. Huisarts en raet. sing: Nignegen: Nijmeegs Uniorsitair Huisarsen Instatudi: 1984

"Rethas JUE. Van Boven CPA, Simulated pationts in general practice: a different look at whe consultation. Ar Med $\$ 1987$; 294: $9 \times 12$

Bosena JDy. Behandeling wan urineweninfectics. TOO 1985; (1): 731-6.

Baselier PMA Acure bacteriele urineweginfectios in de huisartspralkujk [Dissertatie\}. Nijmegen: Katholjeke Universited Nijmegen, 1983 
Chapter 4: Simulatiepatienten in onderwijs en praktijk, een literatuur overzicht (Simulated patients in medical education and medical practice. A literature survey)

Reprinted from Huisarts en Wetenschap, 1989, 32, 366-369 


\begin{abstract}
Since its introduction by Barrows in 1964, the simulated patient method has been used in medical education and for research and assessment purposes in medical practice. Inter-reliability in the use of simulated patients for assessment purposes has not yet been satisfactorily investigated. This yields also to what specifically can be concluded from these assessments. The method is however well documented for educational purposes and for registration of actual procedures in medical practice and its use in these simulations is justified. The simulated patient method offers the possibility of more closely investigating inter-doctor variation in general practice.
\end{abstract}




\title{
Simulatiepatiënten in onderwijs en praktijk
}

\author{
Een literatuuroverzicht
}

\section{JAN-JOOST RETHANS RIET DROP FERD STURMANS YVONNE VAN LEEUMEN}

Sinds de introdactic wan de sinnuhutiepatherryt in 1964 door 13 arrows is deze methode gehruilit in het medisch onderwijs en woon onderaoth en loetsing in de dagelijkse protatijksituatie. De interbetrouwbarharid bij het gebruik wan simulaticpatientern bij tactsing is nog onvoldoende onderzocht. Verder $z a l$ bij toetsing duidelijker mowten worden wát er gecondhdeerd kan worden ait gegevens dic perkregen worden wia simulatiepatiönten. Foor educatieve doweinden ten voour registratie van de feitelijke gang wan zaken in de medische praktijh is de methode ecturer goed gedocuncuteerd en is het gehruk orvan gerechnatardigd. Wome de husartspraktijk bikdt de simalnfiepatiezut-methode onder meer mogeligkheden om het begrip saristie nader to onderzoken.

Retlans JJ, Drop R, Shurmans T, Van Lecumen Y. Simulatiepatichuten in onder-

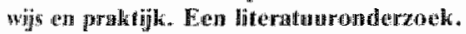
Hustsarts Wet 1989; $32(10): 366-9$.

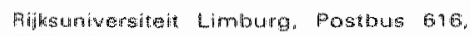
B2G0 MD Matw Etricht.

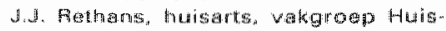
artsgarneskund prof. th. P. Drop, vakproep Medische Sociologie: prot dr. Fr.

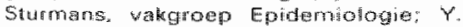
wan Lequwen, hulsents, vakgroop thuisartem geneskunde

Correspontertio: J.J. Rathans

\section{Inleiding}

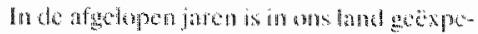

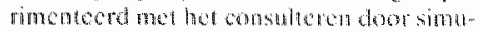

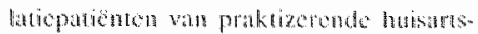

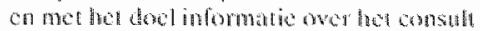

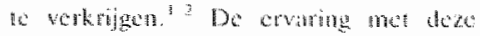

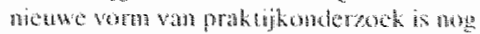
beperki mar polet op het strewen natio meer pratkijligerich onderand is $1 \mathrm{e}$ ver. wachten dat in de thekorish smotationa fenten wather petroukt zullew worden an

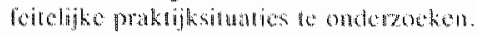

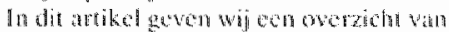

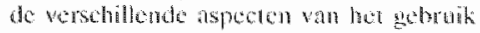

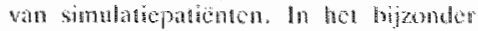
komen twe vagen an we arde:

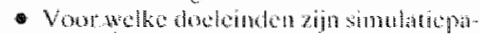

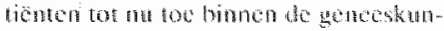
de getrutht?

- Hoe benroublar en hoo valide is het gebruik van simulaticpatitnten?

Woor de beantwootefing wan deze vragen is gebruik gomaka wan ons bestand anin publikaties ower sintulaticpatherter. De basis diaran word geronnd door hed bestanel. zouls dat in nowember 1986 bestond arm dice MoNaster Universitent (Hamilton. Crmm da). destijds toonatangewend op het gebied wan onderzok wan en met simblaticpantientern. Na 1986 thebben wij dit bistand systematisch angevuld met do relevante litentuur.

\section{Barrows}

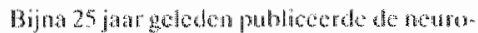

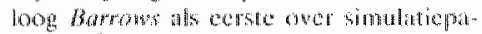

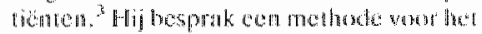

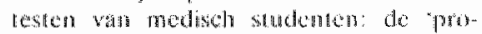

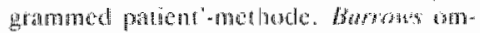
schroef oce "programmed paticht' ats 'aten feck dia een rol als patich heot ingesteacerd on deze rol verwalgens spetelt in een

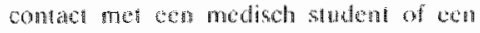
arts'.

Borropy gaf twe ledenen om met pro-

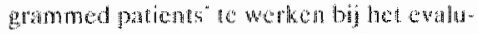
eren war madish sudentem. Ten eersto zijn deze "patienten" gestandardisect, in

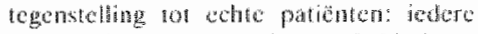

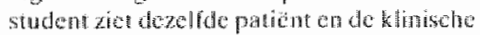
wardigheden van de studerion zijn dardoor beter met akar te vergelikan. Ton

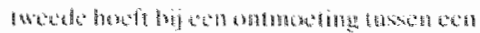

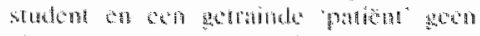

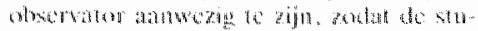
a vanelen?

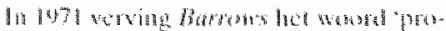

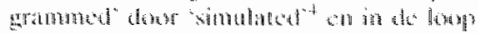

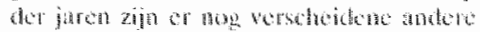

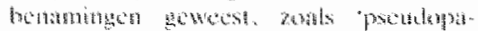

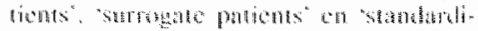

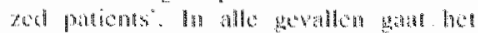

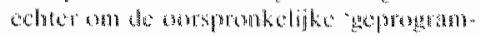

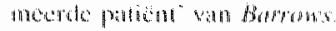

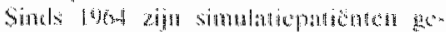

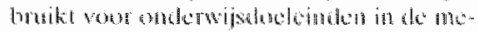

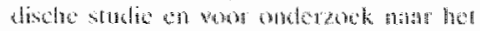

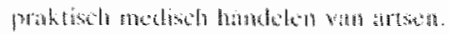

\section{Onderwijs}

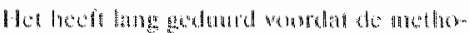

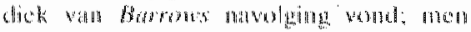

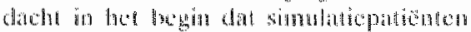

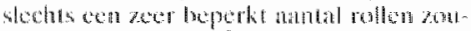

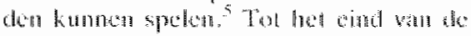

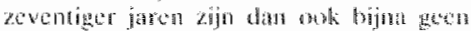

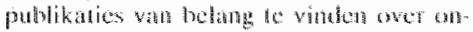
derwijskendege ervatuged med simula

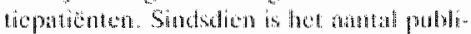
katies echter stok toogenomen.

Bimen het onderwijs hext ale simula

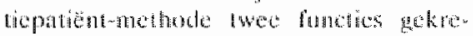

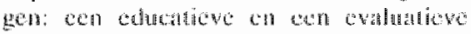
functic.

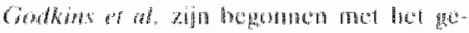

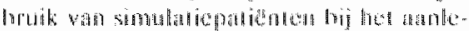

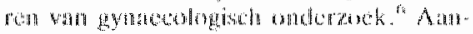

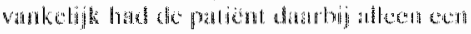

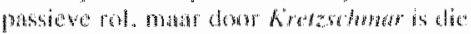

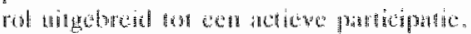

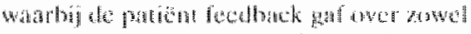

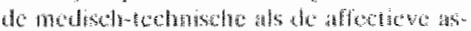
peeten van het gyrabeothenseh onderzok.

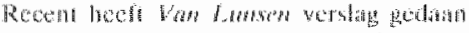

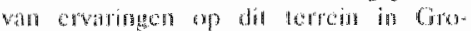
ningen."

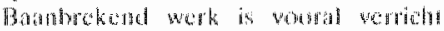

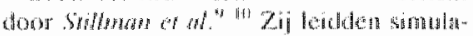

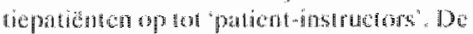

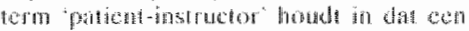

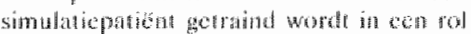

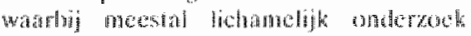

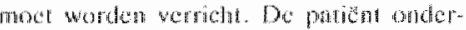


Ook bi het gebruik van simulatiapatienten wom toetsing geld dat simuleutepatienten zowed mogelijk op ectue puliesten moden lifken. Er zijn inmiddels vele studies verrich warin werslag wordt gedan wan suc. waswol gebruik van simulatiepatienten als

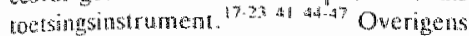
beslabt nog steds onenghed over do vaag wat er nu precies met sirmulat nienten gemeten wordt. Dit is onder andere een gevolg wan het moeilijk miectbars begrip "medische competentie". In dit opzich onderscheid de simulatieparan-methode zich hict wan andere rueetiostrumenten alls geschreven simulaties, multiple choicetoetsen en kennistoetsen. Indicatief voor dexe situatic is dat Nerfeld and Noman in lun bock "Assessing clinical competence" klimische competentie een 'battered child' nowmen.

Sullhran and Gillers vermelden in een overzicht varn evaluaties van klinische competentic wolsswar dat simulatiepatienten in de loop der jaren gebrukt zijn on alle aspecen van klanische competentie te metery - me name interpersoonlijk vardigheden en interview-en probleernoplossingsvaardigheden - maar maken toch niet: duidelijk hoe valide die metingen waren. ${ }^{\$ 9}$ Tot nog toe zujn er geen onderzoken geweest die en anduidige conclusie over dit olyderwerp roesaten.

\section{Beschouming}

Wat kam ma worden geconchdeerd ten

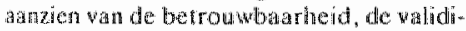
teit en het loekomstig gebruik van de sima latiepatie nt -me thode?

Voor edncatieve doeteinden zujn sinulathepatiènte a een uniek lecrrinddel; met name de studies wan Srilknar e al bevestigen the keer op keer. Het gebruik van simula thepatientep op onderwijskundigaterrein zal dasrom hoogstwarschiljnlik toenemen. Daw zuller wok de mogeligheden wor den uitgebreid, met name door verbetering var simulaties op lichamelijk gebied. Het gebruik van simulatie patięnten voor onderwijkundige toersing cuprak tijkdoeleinder (registratie en toetsing) vereist echter ook mader onderzoek.

Hoewel stillwam et al hebben aangetoond dat "patient-instructors" studenten

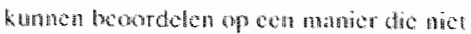
onderdoet von het beomdeten dom abser.

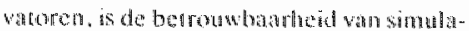
treparichten bij toetsing pas zeer reccus expliciet onderzocht. ${ }^{43}$ Deze onderzocken wekken wellswat vertrotwen, mas zijn nog re gering in atath. Het is datrom an we bevelen dat er meer studies mand de interbetroubarheid xan simulaticpatienter zullen worden opgezet. Er zijn met name nog geen studias verrich die alle aspectem van de betronwbatrheid angelijkertijd onder the loep nemen.

De betrow watarbeid van simuaticpateenten bij het rapporteren over hen handelen wan artsen in de dagelijkse praktijo is beter onderbotwd. In reconte studies hatden simulatieptienten in alle gevalen betrouwbarheidspercentages van meer dar 85 procert. 35 is

De raliditeit van simullatienatienten voor educatieve ren registratie-doeleinden is woldoende onderbouw. Wat betreft de toet sing zal duideligker genatakt moeten worden wh er nu precies word gemeten (büvoorlieeld kennis of socialle vatrdigheden'.

Tenslote de vraag, war simulatiepatienten voor de huisartspraktijk kunnel beteke. nen. Alereerst is en geen ander "instrument" dat zo valide kan weergeven wat zich in de spreekamer afspeeft, en dar bovendien ook andere informatie verschaft dan bijworbeel wia selirfolijke instrumenten kat w worden verk regen. Mricht in de wath tie tussen huisartsen kan beter worden bostudeerd, doordat een simulatiepatien keer op keer dezelfide patient blinft. De huisarts wordt zo de echte afluankelijke waribele. Daarnast kunnen sumatiopanienten in de praknik een uitstekend onderwigsmedium zign bil de opieiding wan assisgenten in de huisartsonleiding.

\section{Dankbetuiging}

Met diank arara drs. Cees wan der Vleuten wan de afdeling Onderwigsonthikkeling on -research van de Rijksuniversiteit Limburg wow zijn watardewollie suggesties.

\footnotetext{
Literiatuer

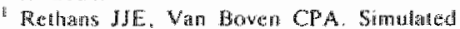
patients ingerenal practice: a differcent look a
}

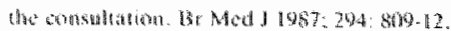

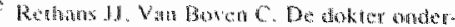

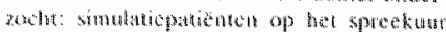

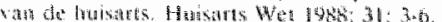

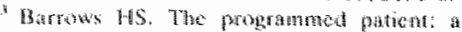
teduryez

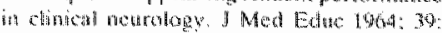
$80 \geq-5$

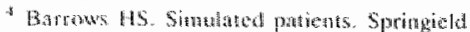
(1). ): Thomas, 167!

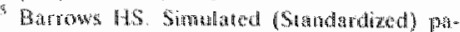

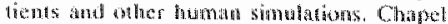

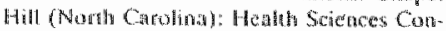
sorrinatist 145

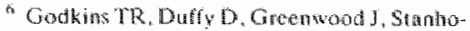
pe WD. Ulitization of siandated pationts to

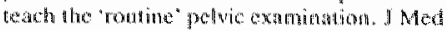
Educ 1974: 49: 1174,

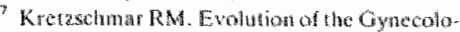

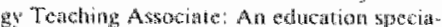
list. Am Joural Obstet Gyactol 1978; 131: 607. 7 ,

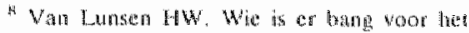

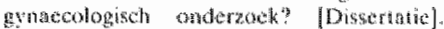
Gomingen: Rijks Linversiteit Groningen 1986 .

* St.ilman P. Ruggill J, Muala P. Sabers D. An

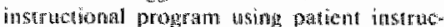
lons as teachers and evatuators. Proc A nem Conf Res Med Edtuc 1979, 18: 53 .8

(u) Stiliman PL, Ruggind IS, Rutala PI, Sabers

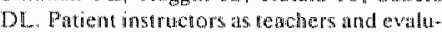
anors. J Med Edtar 1960: 55: 186.93.

it Juhnon BF Pratkonki L. Lamier R. Evalua

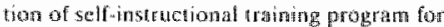
simpulated patients. Proc Antu Com ${ }^{2}$ Res Med

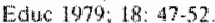

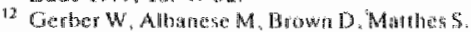

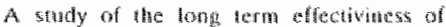

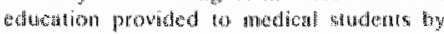

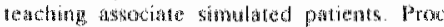
Antu Con The Med Edue 1483, 22:180-5.

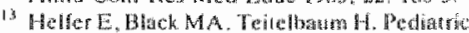

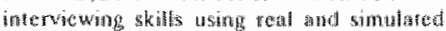

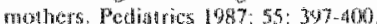

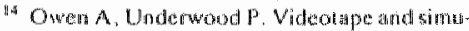

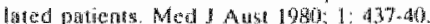

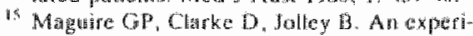

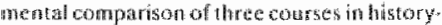
taking skiflo for medical sudents. Med Fdac $1977 ; 11: 175-82$.

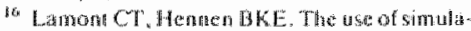

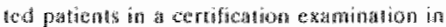

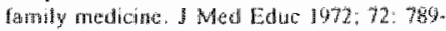
45

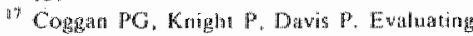

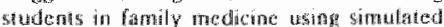

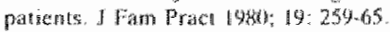

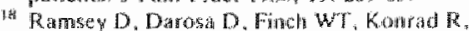
Birich A, Peters W Folse J. Evalantion of 


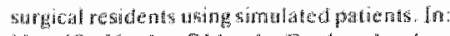

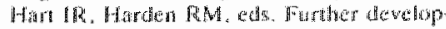

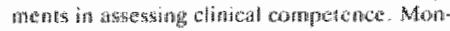

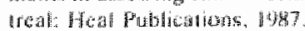

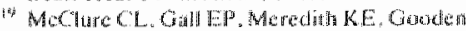

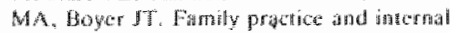

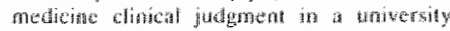

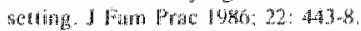

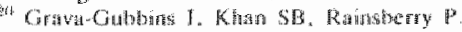

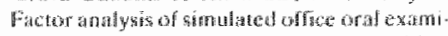

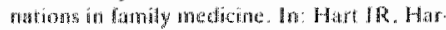

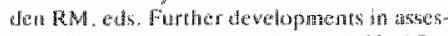

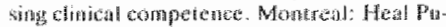
Whathions 1057 .

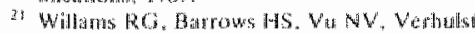

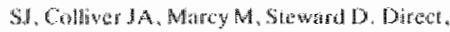

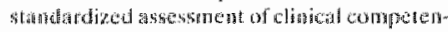

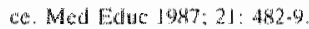

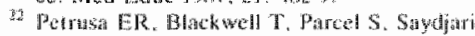

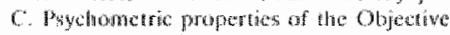

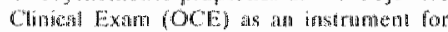
fi:tal avatumenon. In: Hain IR. Harder RM.

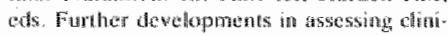

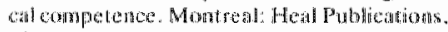
1986

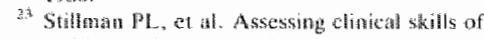
resiblems with standerdixed petionts. ano lot lat Med 1986: $145: 762-71$.

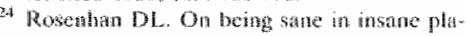
ces. Scienee 1973:179: $250-79$

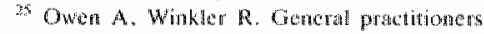
and psychesonal problems: an evaluation

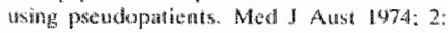
303

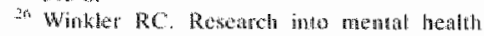

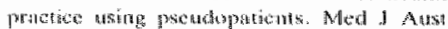

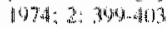

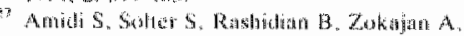

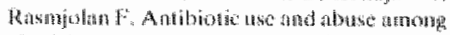

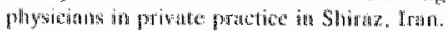

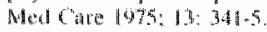

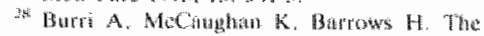

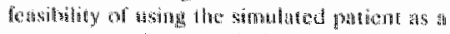
rote

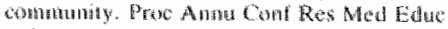
1030,$15 ; 295$

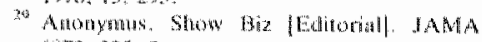
$867 ; 225 ; 56$

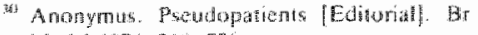
Mcoi I 197: 266: 729.

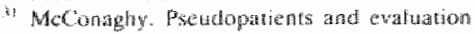

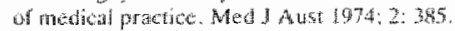

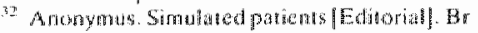

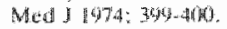

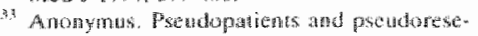

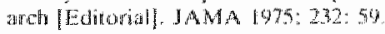

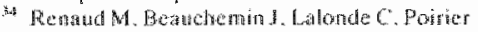
14. Berthitume Patice seting and poseri.

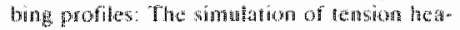
daches togeneral proctionters work big in diffe-

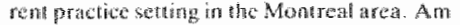

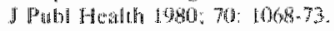

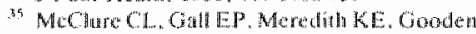

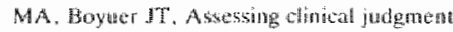
with seandardized patsones. $\mathrm{J}$ wam Prac 1985 $20 \mathrm{x}+457-64$

Th Noriltan Gh. Neafeld Wh, Walsh A. Whod

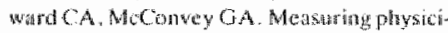
ans" performances by ming shoulated par.

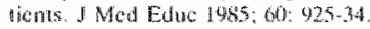

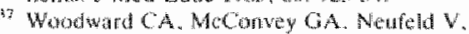

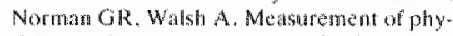

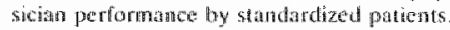
Med Cure 1985: 23: 1019-27.

34 Jolly A. A revien of hastes in live pation simblation. PLET: 1962: 69-107,

Wu WW, Steward DE. Marey M. Mn astas ment of the onsistency and aremacy of sean-

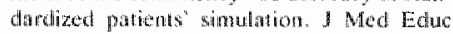
1687?62: 100.2

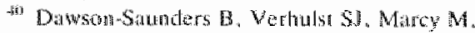
Steward DE. Variabutry in standardized pa-

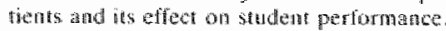

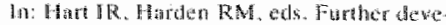

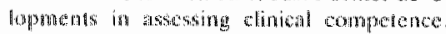

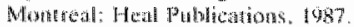

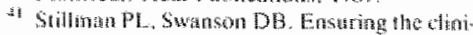

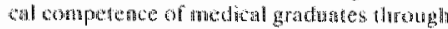

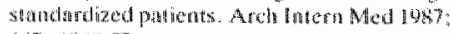
$14: 112+18)-52$

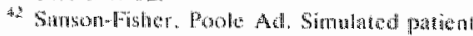

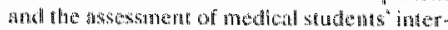

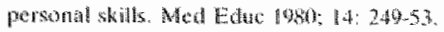

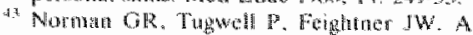

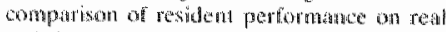

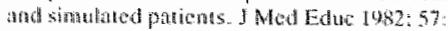

$708-15$

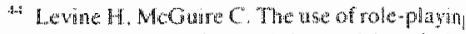

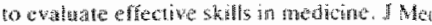
Ex 1970: 45: 704 5 .

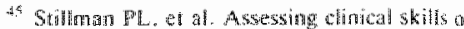

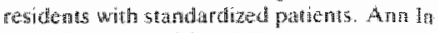
9อ

whe Graff E. Post GI, Drop MI. Valleationo a bew masure of whinal protblen-5olving

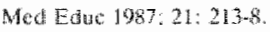

if Smin DH, McWhingey IR. Comparison a

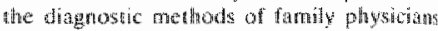

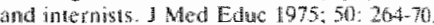

a Meuteld VR, Norman GR. A sessing moneal competene. New rork: Soringer, 1925.

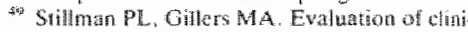

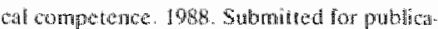
viont.

\section{Abstriact}

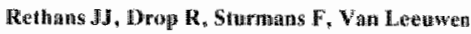
Y. Simlatated potients in medical educathon ant medical prachice. A lineratere survey. Hutsarts Wat 1964: 32(16): $366-9$.

Since its anderdustion by Barrowa in 1954. the sinulat ed paticm mettod has been used in medi

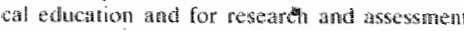
purposes fint medical practise. Inier-melabbility in tha use of simulated patalut for issessment

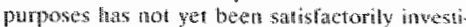

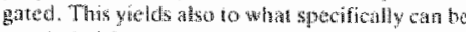

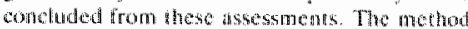

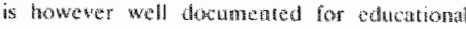
purposes and flor registation of actul pro.

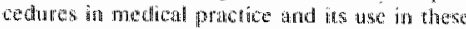

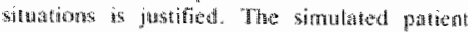
methed offers the possibility of more rowsely

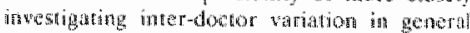
practice.

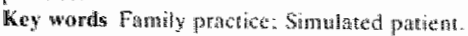
Correspondence J.J. Rethans, University of

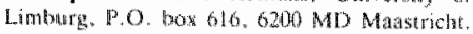
The ventertands. 
Chapter 5: Competence and performance: two different concepts in the assessment of quality of medical care

Reprinted from Family Practice, 1990, 7, 168-174 



\title{
Competence and Performance: Two Different Concepts in the Assessment of Quality of Medical Care
}

\author{
JAN-JOOST RETHANS, YVONNE VAN LEEUWEN, + RHET DROP, CEES VAN DER VLEUTENS AND \\ FERD STURMANS *
}

Rethans $J-J$, van Leeuwen $Y$, Drop R, van der Vleuten $C$ and Sturmans F. Competence and performance two different concepts in the assessment of quality of medical care. Family Practice 1990; 7: 168-174. In the debate about ' what is a competent general prisctitioner?'little attertion has been paid to the actual practice situation of general practitioners. This paper, based on the 16 mast important studies in the literature about medical competence, tries to re-initiate this debate by proposing a clear distinction between 'competence', (what a physician is capable of doingl and 'peifformance' (what a physician does in his day-to-day practice). With this distinction we llooked tht whether situdies defined both competence and performance, how they dealt with these concepts, whet measurement instruments were used and what the conclusions of the studies were. Although it is the common rassoning that competence is a good predictor of performance this concept could not be alfirmed. This surwey shows that the majority of studies use wrong concepts and come to invalid conclusions. With the empirical distinctien between compettence and performance however, this paper proposes new directions for the quality assessment of general practitioners.

The British governments whitte paper on primary healtb care' has again raised the debate about the quality of care delvered by generall practitioners. In this report indicators are mentioned in relation to high guality', such as personal availability, preventive anctivities, attendence at post-graduate courses and certification procedures. Also, a recent series in the British Medral dournal, "What is a good GP", puses scver questions regarding the critera against which general practitioners should be measured. The discussion is closely related to the issue "What is a competent doctor?" "standard messurements of quality" and to assessmetrt procedures in general practice.

What is a competent doctor? Many authors and organizations in areas of modical research and medical edacat ton have struggled with this quextion. With the introduction of standards and logiwal branching flow charts in medical practice the debate about "what is competence", "what is good" and "what is quality" is also of current interest in this a dea. The relewant liter-

"Depart rucot of Gicne

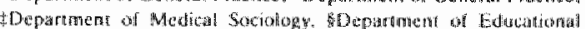

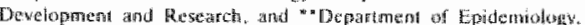

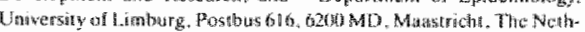

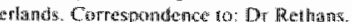

ature includes a large vocabulary, eg: ability, behaviour, performance. clinical judgment, clunical reasoning, dobitual performance, problen-solving, dinical competence and the combination of know]. edge, skils and attitudes.

The fundanental problem of this lack of clatity about competience is that each author means different things when using the word sompetence". Other authors dest with the same issue butt ase different names. The use of different instruments to measure competence, for instance "peper and pencit tests". "chart audit", and simulated paticns", complicates the issinte further

This paper intends to give a elearer picture of "conpetence and specifically proposes clear distintion between it and 'performance'. The reason for doing 30 is becatse we think that the debate allout the quality of medical care will gain in clarity if this distinction is used. After making the distinction between compe. tence" and "performance" this will be applied to at syththesis of the literature examined from several viewpoints: to see if studies definte thoth competcitce and performathe, how the studice deal with these concepts, what medsurement instruments were used and fonally the conclusions of the study. Details of the psychometric characteristics of different measurement 
instruments wit not be examincd. Finally thes paper indieates directions in which assessnent of the gualizy of eare delivered by genters practitioners mightil go. This paper is not intended as a quantiative review of the quatio of care literature but it takes accoun of the 18 unportant studies on this subjact (Table I).

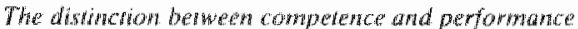
Ouality of care is the crux of every hath care system. In trying to guarante this quallity lieconsure boards have set up examindion systems for medical suidents,

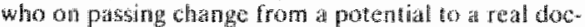
Lor. The reasnong underlving these exminations is that passing them predicts the performance of a doctor in the period after the medical school. In other words passing the examiantions poredicts quatity of care.

Sonior and loyat distingtushed between "compehence' and 'pertomance'; the former meaning "what a physician is capable of doing' and the latter "what a plysician actually does in this day-to-day practice. 'These definitions clearly demarcate the exaramation: before and during the examination the candlidate mast behave to the best of his abititics, he deals with the competence seting. After passing the doctor deals with mormal day-lo-day sirthations and altempts to cope with them; he aldeds wh the performance setting

One might argue that "bormpetence" is made up of several aspects and that it would be more appropriate to speak of competences", referring lo competences as a series of parametes such as diagnostic and theram peatic skills, interpersonal skills, wete. In this paper however we want to crophasize the construct of competeno as a sibgle entity. With the measurement of competence (or performance) both concepts indeed can be operationalized into seweral aspects.

The distinction between comperence and performance atises the question of their relationship. In this there are two possibte approathes.

The first one, more psychometrical approach, considers competence as the limut of one's performance. Or. stated diferenty performanes is an indicator of

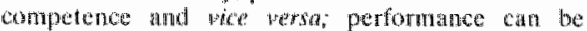
observed wherefs competance chn only be inferred. In this concept competence arat performance are not two separate coneepts but ante separate measurement points of the sanc concept. An example of this restoning is the current examination systent passing predicts pertortatinee

Is the second spproach competence and performance are considered as two separate concepts although they wa be refated in a very delicate way. This approach takes nore account of the actual practice experierces of gneral practitoners. When treating paigents doctors are influewerd by motivational and sifuational wartabes. This siruation is completely different from the examination situation

The fist reasoning is the ane that most educationat ists bave followed and is the busis of all existing exarnination systems.
Whather comperence is inteed at good predictor of performance thas been studied in wight of the eighteen studes mentioned in Table (studies $1-6.9$ and 10 ), all but one fall to establish this relationship. Only perer son concludes that years of experience is an indicator of competence (Study 3). With these results at hand researchers conclude that their meastrement instroments are not good enough. They conclude that the reason for not establisting a relationship between competence and performance is a pxychometric problem.

As in the past 10 years there is only one direction it which this educational researdh line will go: to construct more and refined measurement instruments which try to alssiss competence as closely as possible.

The second ressoning, to consider competence and performance as two different concepts, could be more fruitful. If it could be proved that we have to ronsider competence and performance as different concepts this could give new inpulses for research in to the daily work of physicians. More research could be focused then to the diangostic pathways which doctors use during contacts with real patiersts and which most times do not. seem to be very logicul or fail to reach ideal stasndards."

In order to explore this second reasoning the afore mentioned 18 studies are rewiewed to look at how atuthors dealt with comperence and performance. We will adop: the definitions of competence and perform. ance by Senior and Lloyd and apply these to the literature.

\section{Definitions}

Only three of the studies in Table 1, give an explicit definition of cither competence or performance or both (12.13 and 16). Fabb states that competence is to assest periormance" (13), whille Morgan seas a competence level as neccssary for adequate performance (12). Only Neufeld explicitly separates both concepts in their definitions using the same theoretical concepts as we do in this paper (16). The 15 other studies do not define competence or perfomance other than in terms of a measurement instrumen. Careful examination of these 15 studies, however, shows that most authors implicity use different concepts of performance and/or comperance.

Berause of this implicit use of concepts the assess ment of these concepts thad to be done in two steps. First. we looked how the atuthors used the terms performancetcompetence and whether they separated both words or used them athernatively. Only one of the 15 studies implies the cornpatence concept (3). Of the 14 other papers eight use the performance concept onty $(4,5,7,8,14,15,17,18)$. Six papers use both concepts synonymously $(1,2,6,9-11)$, (Table 1, subheadings $4-6$ ).

In the second part of this assessment aut defintions of performance and oompetence were applied to all the suludes to see whether these were in accordance with 


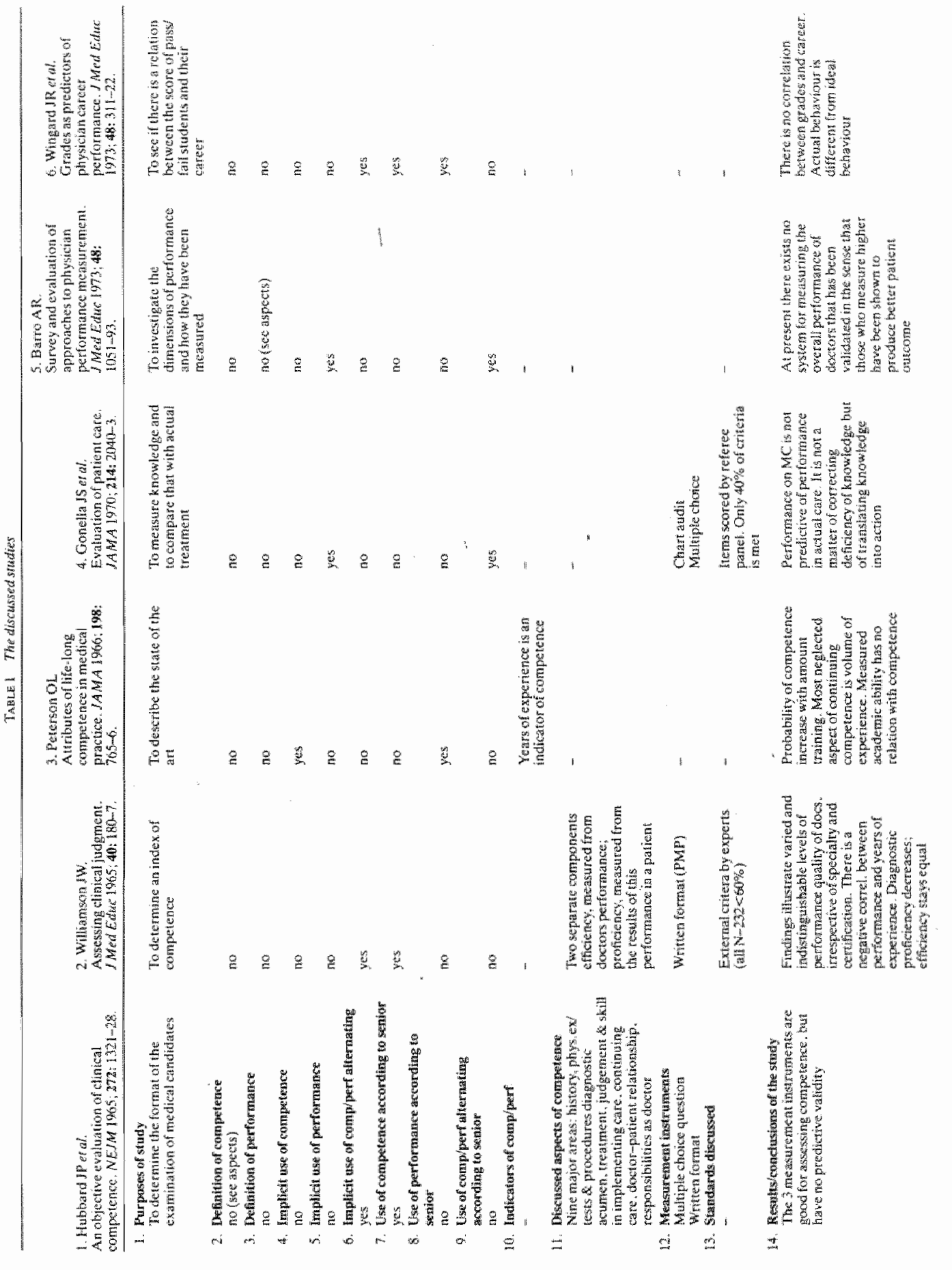




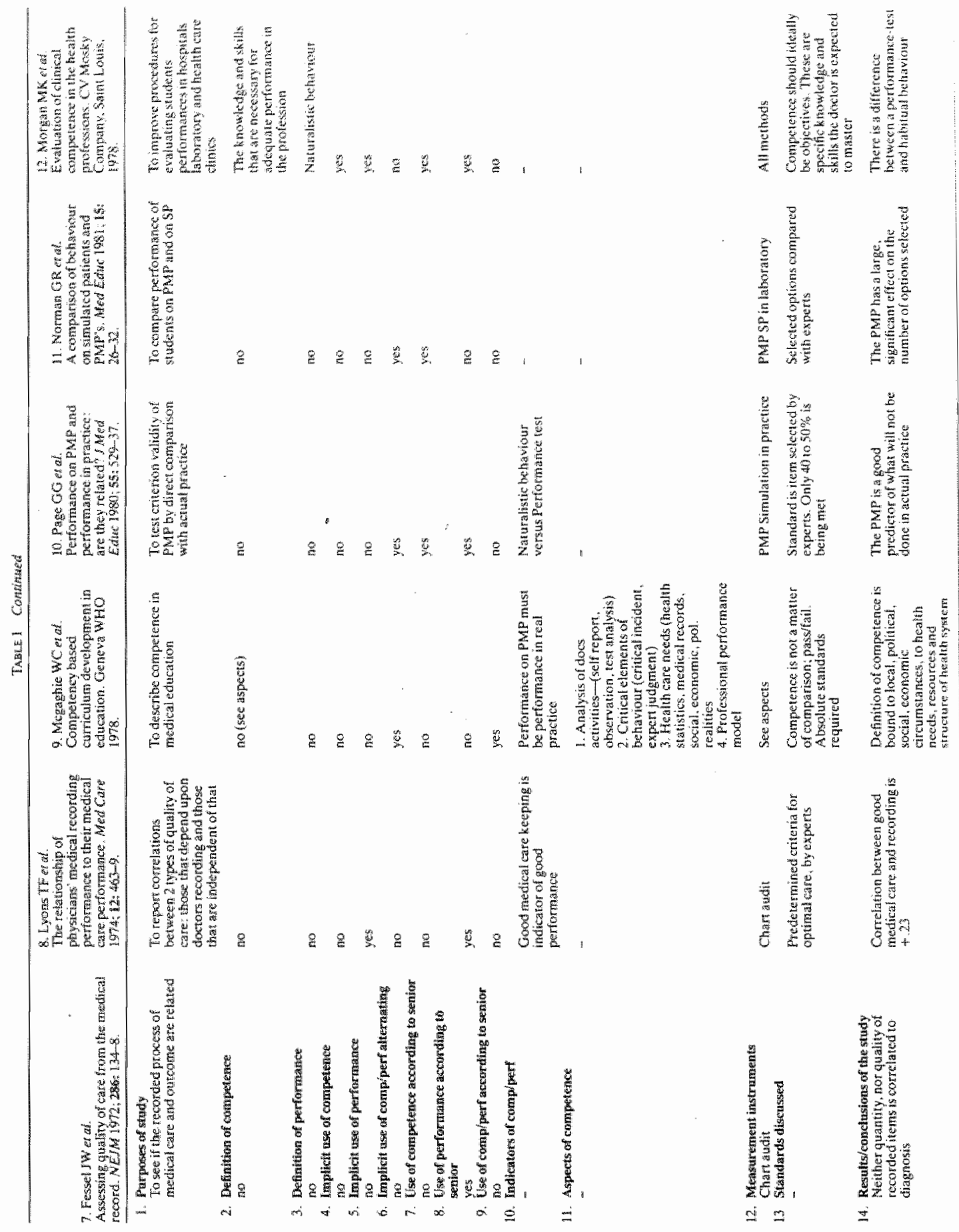



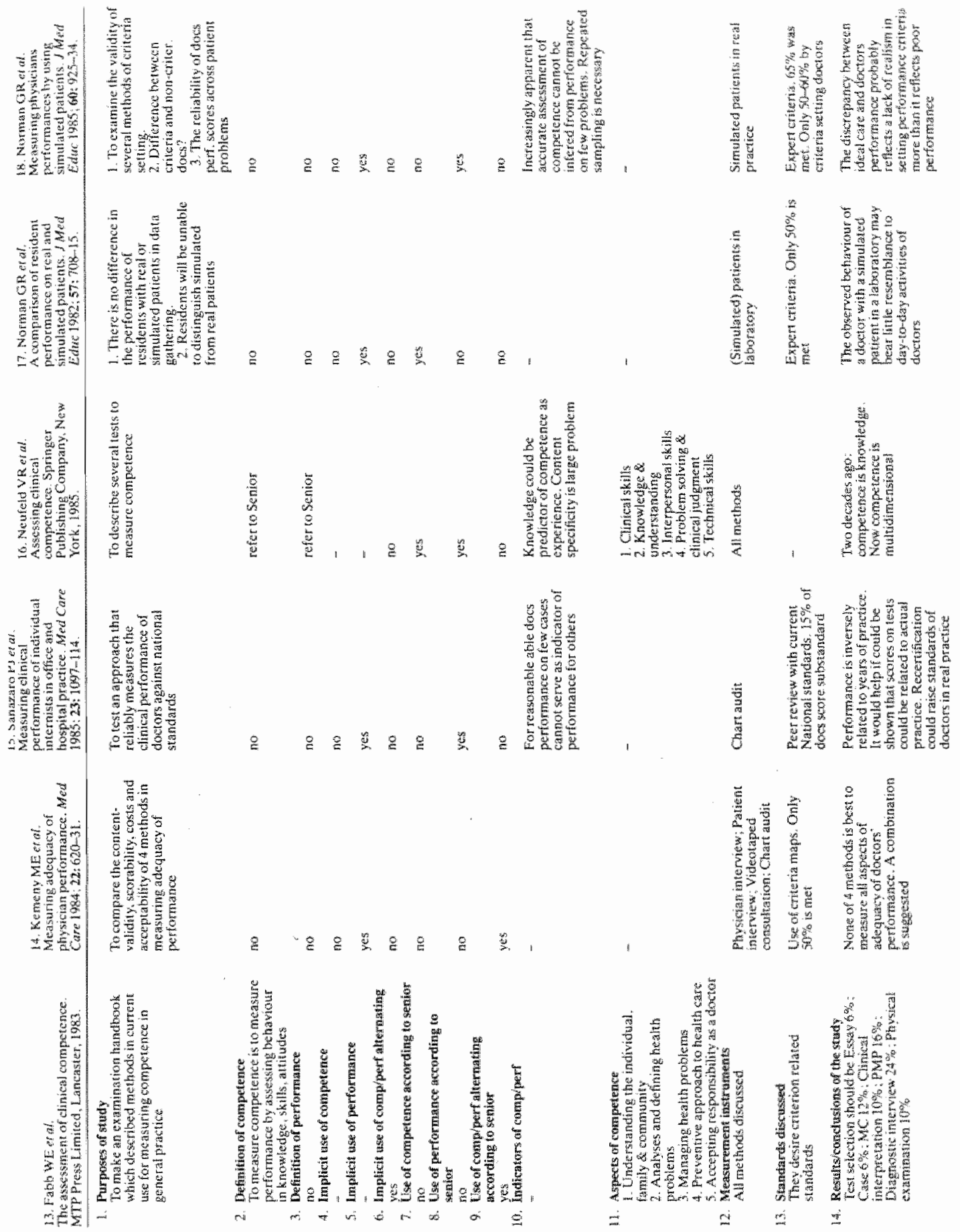


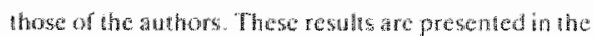
subhoralings $7-9$ of "Table 1

If appears thet in 10 of the 18 gapers the deforitions alescribed by the duthors are wathen with ours (1-6. $16,11,14.17)$. With this result an hand we concleste therefore diat four situdie describe only competence $(1,2,11,17)$. Five stadice deal wh performance $(3,7$, 8. 15, 18). Another four papers deat with both con-

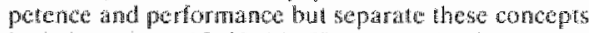

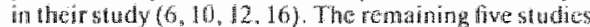
we both conceph alternatively and withou distenction $(4,5,9,13,1,4)$

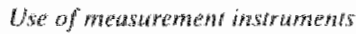

Intarently ralated to the use of competence performance concept is the sige of meatsurement instruments. It is argued here that the distinction between corrapetence and performance hat its implications for the use of meastrement instruments. The objech of study (competence, performance) find wsed measurement insintnuest do not math. then vesedreh conclusions ate invallidaced.

Measuremene instruanemts (or methods) can be diwided into clinect and indirect mothods. With direct raethods the research workers seo or hear a physician ferling with paticuts or with exanyiners. Such mothods inch de observation of doctors (both wiclea and at dio), use of standardized patiens or oral examinations. With indiect methods direct obserwation of doctorpatient contacts is not possible. They depend upon written simulation papers, chant audits, interviews and muleple thoice questions. Using the conceptro of competence and performance it is clear that some instruments nay be used in born concepts, while others may not. Written simulation papers, laboratory observation, orat examination, multiple choice questions can only be used in the competence-situation. Chare atdit can be used for performance measurernent. One of the best nethods for penformane masurement (high reliability and watidity) is some kind of unobtrusive ancisurement stat as the use of standadized patients in a blinded way, so that doctors do not know when they are deathag with such in pattent. We analysed 16 of the 18 studies (3 and 6 ande review papers) 40 see whet the the appropriate mestatement inst rument hat been used. For wample if an atubthot used a writen simulation anwhod and yet latelled the stidy as "mostung geriomance this is considered incorten. Five $(31 \%)$ used incorod instruments $(2,5,9,14,17)$ or labelled their instuments incorrectly. In these five studios llie authors" purposes were to describe performance, but unstead they measured aompetence.

\section{DISCUSSION}

The paper shows flat rese wroly workets do not specify wery carefully which concept of competencep perlomance they mestr. Onc might angue that athors do not give defintions because it is neither important nor meressary. Howener becaluse every athor use differ ent conepts implicity, a defintion is imperatue and enables the reader ro interpert the situdion he is degating with.

The comparison berwen the atothors implict defiwitions of perfonnancefcomperence and the way we chassified these acoording to our concepts revealed a distubng pichure. It is disapponting that so many papers fall to delineate what they are dealing with and it is even more disturbing that most aw hors deat with concepts whath they label incorrecty. The conseGuence of this migclassification is also shown in the use of measurement irhstruments: Even among the 11 studies in which the right instruments were used four authors intorpered their own concept talsely $(1,4,10$. 11). This means that in ond seven $(44 \%)$ studles $(7,8$. $12,13,15,16,18$ ) the right instrument matched the right concept?

We are inclined to ensider only the conclusions of these seven studies as wald. Fessol (O) and Lyons (b) conclude that they did not nind a significant relation betwen chart audit and good medical care. Fabb (13) and Neufeld (16) giwe a frame work for a test to measure competence. The other studies. Morgan (13), and Sandizaro (15) and Noman (18), reter to a possible distinchon between competence and performance. This is in line with our reasoning. It 15 clear that the majority of studies adhere to the reasoning that competence is an indicator of perrormance. But as we stated carlaer, all but one fatled to establish whis relationship

To develop a beter empirical basis for the debate about the quality of medical care future studies should concentrate on the difference or relation between competence and performance. The inplication of the results of these future studies nigh have great impact both on medical schools and nedical practice. Will it still be justifed to teach medical candidates compefence' or should medical schools concentrate more on performance"? Sibley. in a study to determine the affects of continuing medical education, concluded that ahbough the purineipants showed significant gans in thair knowledge, they did not cllatage in their performance." Normat showed that doctors in actual practice performed considerably hetow the criteria developed by themselves. Should standards of quatity of medical care. delivered by doctors in their denity practice, continually be used based on competence" stindards?

To find an answer to these questions we think the recommendations are essential. Firsty, researchers should make a cletris distinction between "competence" and "performanee". We think this could be done according to our definitions. Secondly when using measurement instmunents research workers should choose their instrument in correspondence with the object of study. Ingeneral it can be stated that different concepts reauire difterent measurement instruments. For lhose instruments which seem appropriate for both competence and performance settings researciers 
should carefully describe in which setring they are used during the sptudy. Ar present we are running a study in which we try to find more empirical evidence for the distinction betwen competence and performance. We compare general practitioners in three settings. In the first setting doctors will be wisited by standindized patient, indistingurshable from real patients. In the sexcond serting the same doctors will deal with the sarne standardized patienss in a laboratory situation (video controlled?. Finaly the doctors will handle a written simulation about the same patients as they med before.

Other researdi questions in this ares are whether a relation between performance and ouput of medical care exists. What factors infuence the perfomance of doctors and how may they be infuenced to taise the standards of performance?

It is our conviction that the debate about the quality of medical care can take place at a higher level if these fundamental questions are answered clearly.

\section{ACKNOWLEDGEMENTS}

The at thors wish to thank Dr Robin Hull (Birningham), Pie Hobus and Wim van Zutphen for their conments on earlier drafts of this paper.

\section{REFERENCES}

Secrearies of State for Social Services, Wates Nonthern fretand and Scotland. Pronowing better Meath Londer: HMSO. 1987. (Cmind 249\%

Gray DP. Assessmentingeneral practice. I $R$ Coll Ger Pract 1088,$38 ; 34-345$.

Sonior JR. Towards the meatsuemert of competene of medicine. Phifadelphia: National Board af Meaical Examiners 1976

LLoyd MS. Defintions of competene in spectallics of medi

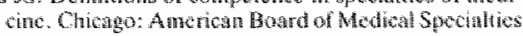
1979

Rethons JJE, Van Boven CPA. Simutated paticats in gemeral practice: as different look at the wonsulfution Ar Med $J 1987294: 849-812$

th Sibley IC. Sackett DL, Neufeld V, Gerrat B. Rudnick KV.

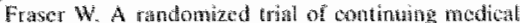
education. New Engl I Mod 1982,306:511-515

"Norman Gik Noufeid VR. Wulsth A. Woodwited CA. McContey $G$. Measuring physiclans" performances by

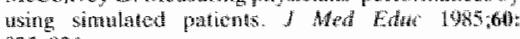
$925,94$. 


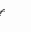


Chapter 6: A method for introducing standardized (simulated) patients into general practice consultations

Reprinted from the British Journal of General Practice, 1991, 41, 94-96 


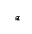




\section{A method for introducing standardized (simulated) patients into general practice consultations}

\section{JAN-JOOST RETHANS}

\author{
RIET DROP \\ FERD STURMANS

\section{CEES VAN DER VLEUTEN}

SUNMARY. A study has been undertaken to determine whether it is possible for a set of standardized (simulated) patients to visit general practitioners, without being detected in a heaith care system where doctors have foxed patient list's Sirce sending standardized patients inco docHors offices is a new way to assess the performance of general practitioners: this paper describes in detail the methodology that has been used for visits.

the paper looks first at the general preparation for visits and secondiy at the spectic preparation concenting the fine detail of the individual wisit. The method was tested in 156 consultations with 39 general practitioners and in no cases where the stumdardized patients detected. None of the doc. fors visited felt offended and all were prepared to cooperate in futwe studies with standardized patients. it is conchuded that the standardized patient method, following the slepby-step procedure described, is reasible in actual practice.

\section{Introduction}

D ECENTLY the need tas been stressed for new mathods of Rassessing the actual performance of general practitioners Fatrer than assessing what they are capable of doing: their competence. . $^{2}$

Doctors' behaviour may be measured directy of indirecty. With direct methods the research worker observes the physician dealing with patients by means of video- or atudo-tapes, or the use of sandardzed panents. Inctirec methods consist of chan audits or written or oral examination. The choice of a particalar arehod depends on whether one is interested in actual practice of doctors or in test (comperence) situations. For both situa. rons bigh walidity and bigh reliability of a method are essential.

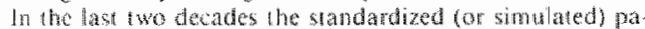

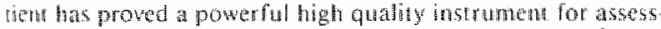
my the competenew on medieal sindents and dociors. "This method has been extended by intoducing standardized paiches

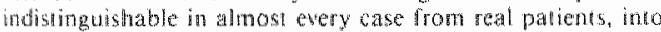
general practice. Since the method is a direct one and both relliability and validity are high, "in may be the best method of wssessing dinicul care Experience of this method in achal prato lice is limited, only lour wudics naking valid, reliable use of sandardized patheris in practice hawe been reported." Woot wat described the we of sandardized patients in the Nonth Anenican stuation. "In vicw of the need for more methods for assessing gerformance is actual practice, and in wiew of the lack

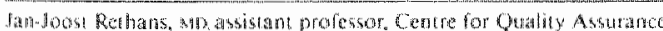

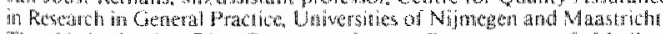
The Nenterlands: Riet Drop. professon, Deparmencan of Mcdical

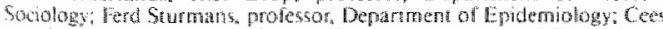

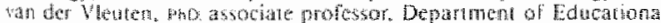
Development and Research, Onwersicy of Limbung. Wassiricha, The Netherlands.

Subruited: 16 Mag lago; accented: 4 September 1990.

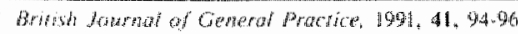

of experience with the use of stand ratized pations in nomat prat. wore, the deparment of general practice ac the Unversis af lim-

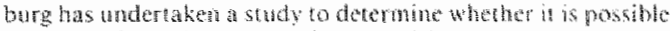

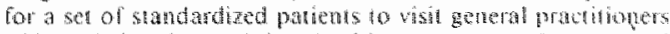
whont being detected, in a bealih are sytem where gentral

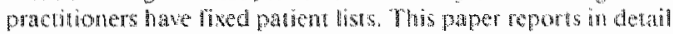
on the method that has bern ased.

The preparation for visics combisted of: firse geveral prepara. tion in which the whole operation was organized regatedloss of

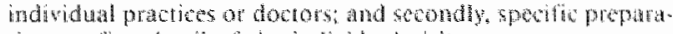
cion on finc detail of the individual visid.

\section{General preparation for the visits}

\section{Pretiminary maling of informanon to doctors}

It was importanto to explain this pescarch as a metrs of studying what happers in the consultaton so that docens did not perceive standardized patients in be offensive on intrustre. The purpose was not to look for 'rutten apples' but to show that krowledge of what gertly happons in the consuhation improves our understanding of why docors behave as they do Doctory were paid for their care of the standardised patients and all dats wats theated in strice confidence and analysed hine. Feedbath was provided to individual doctors and consisted of an inen Higt.

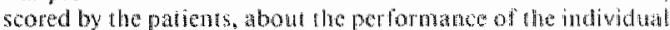
doctor, compared with his on hor perers. This procedure enstived that none of the participating doctors apertented sthical problems with this yype of reseamt in genteral practice. Doctors did not know when or how often they wowd be visited by standardized patients. Information was sent to doctors at the start of preparasion thus ensuring a long interval between consent and the visit.

Doctors were also sent 'detecrion forms' which they we asked to use to report suspected standardined patients, giving the patient's name, the date of the visit and degree of the aloctor's certanty of having identified a standardized particnt.

\section{Selection of simulated medical problem}

Any medical problem wihoun physical signs, and many cond. fions wh physical signs, can be sinulated eflectively. For ex.

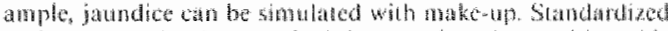

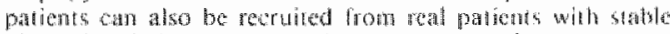

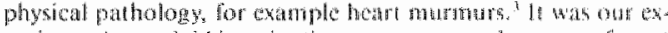

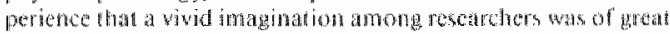

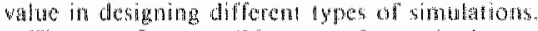

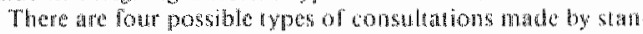

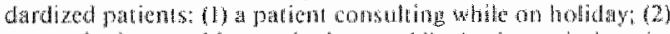

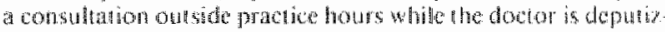
ing for others; 13 a consutation owng nomal work ing hours

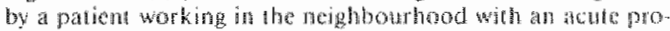
blem (for axample west pain or foreign body in the eye) (4h

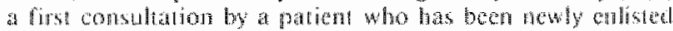
in the practice.

An additional wariant is the home visil bat this has not been insed hiere.

\section{Serding up backgroumd data}

Many genetil practioners will as as the name of a patert's

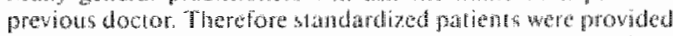
with rames of doctors who lnad been botefed wh written descriprions of the standardized fat iene sole so that they cond respond to at request from the ather doetor. 


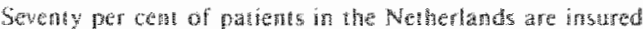

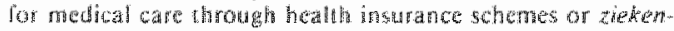

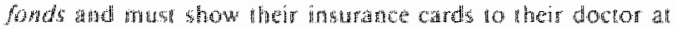
wach wise, These mserance cardis show the private neathe in-

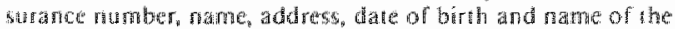

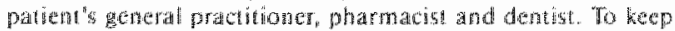

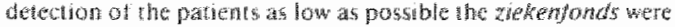

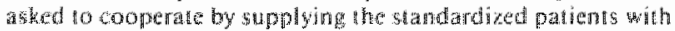

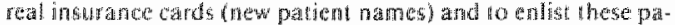

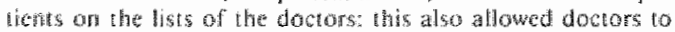

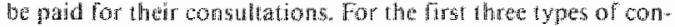

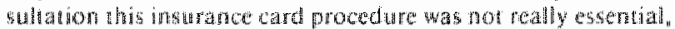

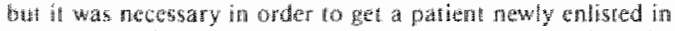

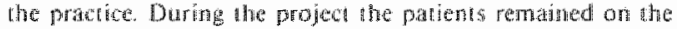

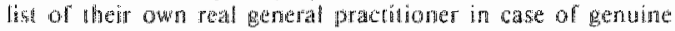
illiness.

\section{Selecron of stondardized patients}

Any moniwad persom may become a standardized patisnt and mathy medioal shools have pools of siandardized patients avaliable for eductional purposes. "The standardized patents used in our stuchies were selected from a pool of 100 in the skills laboratory of the medicat school at Maratrichn. They were paid tor hlatir participaton. The criteria used in selection were that standardized patients mathed the rolss assigned to them (a 40-year -old woman canno be simulated by an 18 -year old man; were able to memorize $20-40$ items of information: were able locope with stress sul everts; ideally possessed a driwing licence and were independent of public dransport (it is unconvincing to be very late tor an appointment when the patient supposedly lives close to the practice).

Standardized pat iets were requined 10 make a writhen tuder. laking to keep all in formation about doctors stricly confidential.

Though some standardized patients were atble to visit ap to 20 doctors in a given role mosn managed 11 and it was found very useful to have some rescrves in case of real illness.

\section{Role and reliability raining}

The standardized patents were trained to play their role as a patien and to report reliable and valid facts about the consulatation. This traning nook place at the medical school and lasted about 14 hours. During these sessions the patients repentediy played their role in contact whts other doctors. With the wse of widrotaps of these sessions the patients were stoppled with feed back about how they performed. To check the reliability anong the standardiged par iens the videotaped test conswitations were

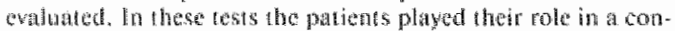

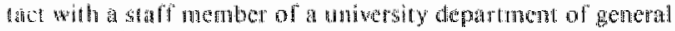

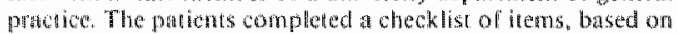
a consensus set of standards of eare (ste the accompanyine paper " itwediately affer such a consul taion; the same consabation was atsu sconed by three independen dochors. For each

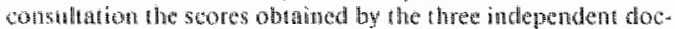
tors were considered to be the gold standard for that particular consultatom, Subeduenty, the individual sopes of the stan. dardized patients were conparred with this gold standard to assess their relabilay. To assess the consistency of individuat patients anch pat ient was retested affer about six neeks, after the visits to the doctors by scoring the sarta consultintion rewaded on videotape. These procedures hate been comied ont suceessully helone she rethability and consistericy agrement scores all fanged from 0.9 to 1.0 (kappa $0.8-1.0$ ).

\section{Specille preparation for a visit}

\section{Suldection of docrors to be nisited}

In some crases economic considerations influenced the selection of a doctor from the list of those willing ro participate. It was

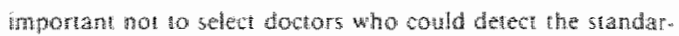

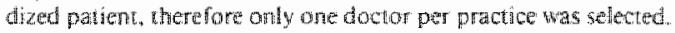

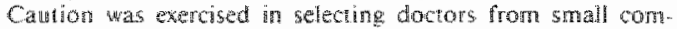
munites where everbody knows arerybody or colleageres of the

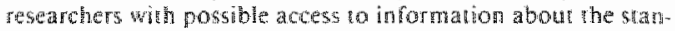
dardized palien "s medical problem or date of wist. Standardired

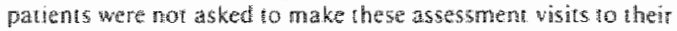

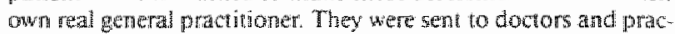

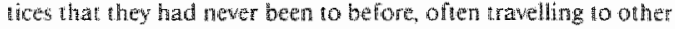
cites. This minimized recogrition of the standardized patiens by practice stare on on har patients.

\section{Recomoing of practice and district}

Al seltected pratices were wisted by the first author to gain knowledger that would make the stories of the standardized panients credible. This cnabled information to be collected about possible addresses for the parients, abour places where the paremts could be employed and about some local interesting buthlings of werts.

\section{Selection of home addresces for stanchardized patients}

The address used by siandardized patieng needed to be credible to the foctor being consulted yet not so farmiliar that he was capable of recognizing in falsity. Adutresses were selected in three ways. It was sometinges possible to access the computer of another doctor with a practice in the same city where the ad. dress of a reat patient could be "borrowed". Real addresses of friends and relatives of the researchers were also used. If these did nou succed a risk was laken in selecting a nor-exusting house number in a known street. Once names and addresses. were selected the doctors were noified by atrine mailing of the ziekenfonds of the inclusion of the patientl on their list. This notification was made between wo and five months before the standardized palient wisted the doctor.

\section{Information given to standardized parients}

Standardized patiens wers given deatled information about the practice to be visithed, such as telephone number, appointment system, address details of the doctors and other gtaff, the number of medical secretaries and the other thealth personnel fphysiotherapisn, practice murses present. Some practices receied students or tramees from the research workers madical school. Where possible standardized patians were cold of the experiences of other standartized patiemts who thad made previous visits. It might be argued that most real patients are nol usually so weli in hormed about a practice, but it should be bome in mind that sandardized patents had to concentrate on their role Every item of information about practices heleded then to feel 'at home' in the practices and enabled then io concentrate better on essential inems of their role. Around six hours of additonal traning was neaded to give this information to patients about the ptactice they were going to visit and for teturn meetings around the time of and afier the accual visits to the general prattioners

\section{Selection of alares for visits}

Afer studying detalls about the aractices the standardized par thents were asked to wisit the practices as soon as possibe, choosing a date which would ensure that they saw their target doctor ather than a deputy.

\section{Pirot wisits}

Alter the raning progranme standardized paients made a single pilor visit - that is, their first real wisit in achual practice as a standardized patien - and this was cwaluated at the medical school. These pilot visits were always stccessful and increased the confidence of the standardized patients. 


\section{Feasibility study}

In Jafuary 1988 all 422 general practitioners working in the pto. vince of our unversity recenved informaton about this study and were asked to give ther writter permission that they would accept standardized patients into hel practices und January 1991. The doctors were not told how many times and when they would be visited, nor were they intomed about the conten of the nedical complaines. The doctors were lold that at the end of the project they would reweive information about whoh stan. dardized pathents had visited them. Of the 442 doctors, 37 agreed to participate and were sent a 'detection form', which had to be returned inmediately after they thought they had detected a slandandized patient.

Of the 137 doctors who agreed to participate 39 were seleted. The main criterion for including a general practitioner was that the disfance berween his or her practice and the uniwersity was les: than 30 kilonetres. This was done for financial reasons. Esch selected general practitioner was wisited by four different standarkized patients presenting four different medical complaints during a four monh period starting at least 12 months after they agreed to participate.

None of the standardized patients was detected. Two doctors returned a completed detection form but both forms reported real patients.

\section{Discussion}

The ethical problems of sendirg standardized patients into dostors' offices were discussed at the start of this project with representaives of the Dutch college of general practitioners (Nedterlands Huisartsen Genootschaph. It was concluded that there were no ethical problems if doctors gave a written consent to be visited and if they were informed at the end of the project when and by which sandandized patients they had been visited.

It was encouraging that in none of the 156 consultations was a parient derected, ewen though each doctor was vishted by four patients. There are probably several reasons for this success. First, there was a lengthy period bet ween the doctors" consent ro participate and the actual visits. Secondly, the original health in surance cands certainly helped the patients to validate stueir position. In ai least two wisits the patients thought that the doctors were suspicious at the start of the consultation. In both cases the original insurance papers convinced the doctors that nothing was wrong. Thirdly the standardized patients were stoplied with a grear deal of inside information about the practice they were going to wisut. Thus, before a particular visit, the only thing the standardized patient did not know was what the doctor looked like. During the project the standardized patients also learned to adopt strategies for dealing wh the doctors' sectetaries. Although we had thought that it would be difficult ot standardized patients to visit doctors in small willages without detectonn all eight such consultations strcceded. The selection of the practioes may perhaps have been too strict. It can be conchuded that for assessment purposes the slandardized patient method is a feasible and adequate method, even in a heallin care system where doctors see only pattients who are registered in ther practice.

There aro some additional aspects of standardized patient studies whith are worth mentioning. The method was not expensive: the budget of this project (lasting (wo years) was calculated to be about 132300 . This sum includes the payment and twinting of the patiens involved $(11100)$ and the fees for the participating dactors ( $\mathbb{f} 320$ ). The rest of the budiget consisted of the salary for the researcher (one doctor working fout days a week).

Marny non-medical issues were reponted spontaneously by the standardized patients. For example, at the beginming of their Wisits most standardized parients felt embarrassed to find that the doctors were really interested in them. Some doctors used to explain where the patiems could find the closest pharmacy or started small ralk with the patients, after the complaint had

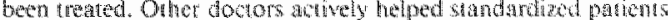

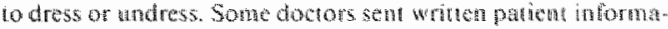
tion about complants do the addresses of the pathons. Some doctors, after rectiving the rout inge maing list of nigu pathents.

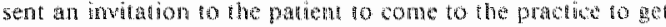
zucquanted. Some peculiar detats were reported: onc doctor 5larted to simg for his pantent and another doctor snoked during the consultation. Furhermore, ench patient saw many watiog rooms and differen styles of inceriod. The whing thes differed considerably. In some cases standardized pactions lelt satisfed with the doetor, but not with the ptactice murses. Somo patiens thataked us after the sudy for enabing them 10 get this experience and spoke of "hose mice, kind and dear dowors.

At the end of athe studies we intornted the participating general practioners about the nethods used. None of the dowtors folt offended and all were prepared to cooperate in future sobitres whth standardized patients. All participathe general practitonters expressed thein betief in research by means of standardied pat tuens in real pratice they considered the data to be walld wh very useful for feedback purposes.

We conclude that the use of standardized patiens is a method which has proved feasible in actual practice. The timitations of the method are in the sinatuation of particular medical problems, as we pointed out earlier. Roles in which patierats would be at risk of undergoing invasive unvesigations wo thalso be an pro. blem. In general practice, howewer, this rarely occurs and if $p$ at tients are referred to hosputals ro undergo such investigations, the results can be simulated 100 . Until now an important ansed of this type of study was to test whether pattents would swoced in entering the practives. This is not a problem and nore attention may be pad to more important aspects stith ats the content of consultations and personal working silyles of doctors. We believe that the standardized patient method has enor. mous potential for research and audit because it gives more insight into what goes on th the consulting roon of doctors. One example would be a study in which doctors were asked immediately after a vist by a standardized patient why they acted as they did. From this we could learn more about why doctors act as they do and evaluate how they provide their cater.

\section{References}

1. Maguise P. Assessing dirical compentence. Br Med J 1989; 298" 4-5.

2. Newble DI. Assessment of dintull competeace: silate of the

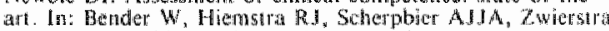
RP (eds). Teaching and assessing chirical compertence. Gromingen: Bockwerk Publications. 1990

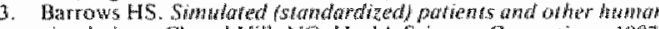

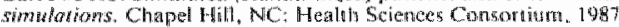

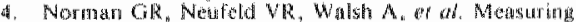

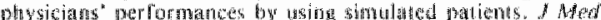

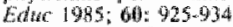

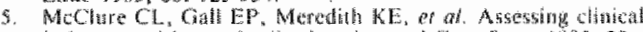

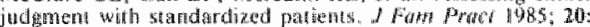
457.464.

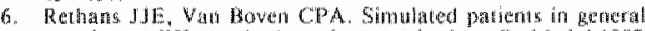

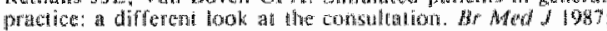
294: 0090

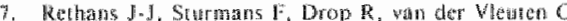
Assessment of the performance of genteral practiolioner's by the

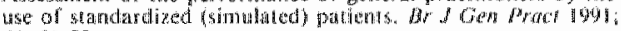
$41: 97.99$.

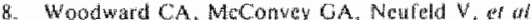

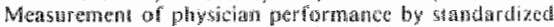
pat ients. Mad Care 1985;23:1919-1027

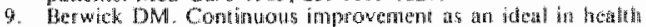
care N Enol I Hed 1989:320:53-56.

Acknowledgements

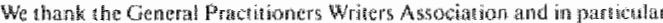

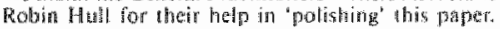

\section{Addresta for correspondeme}

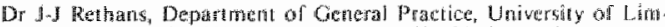

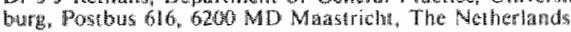


Chapter 7: Assessment of the performance of general practitioners by the use of standardized (simulated) patients

Reprinted from the British Journal of General Practice, 1991, 41, $97-99$ 


\section{Assessment of the performance of general practitioners by the use of standardized (simulated) patients}

\author{
JAN-JOOST RETHANS
}

FERD STURMANS

RIET DROP

CEES VAN DER WLEUTEN

\begin{abstract}
SUMMARY. A study was undertaken whereby a set of stan. dardized 'simulated patients visited geneval practitioners without being defected, in a health care swstem where docfors had fred patient lists. Thirty nine general practitioners were each visited during normal surgery hours by four standardized patients who were designed to be indistingusable from real patients. The objective of the study was to see whether the actual performance of general practitioners, as assessed by standardized patients, met predetermined consensus standards of care for actual practice. The patients presented standardized accounts of headache, dharrhoea, shoulder pain and diabetes. The mean group scores of the doctors on the predefirned standards of care for the different camplaints ranged from 33 to $68 \%$. The results shaw that standardized patients may be the method of choice in the assessment of the quality of actual care of doctors. It is hypothesized that the substandard scores of the doctors do not reflect inadequate competence, but are a result of the difference between competence and performance.
\end{abstract}

\section{Introductions}

TN the current debate about the quality of the performance lof general practitioners, problems arise in defining the methods for assessing auality of practice. 'Good methods are those which possess thigh validity and high reliability, but in real practice the feasibility of a method is also an important aspect. Traditional assessment methods lrave relied on written tests and clinical examinations but doubes have beer cast on their validily and reliability ${ }^{*}$ Audit of medical records has been criticized for its low reliability in assessing reveral aspects of a consultation. ${ }^{3}$ Aladio- and video-laping of consulations are methods which have boul high walidity and reliability. A disadvantage of these methods is that the researcher cannot control which patients enter the surgery room, making, it diffucult to compare performance between doctors. This is not a problem with the standardized (or simulated) patient method and therefore this method has been described as the best one for assessing the management of patients by doctors. With the help of standaraized patients it has recently been shown that doctors in real

Jan-loost Rethans, Ma, assistant protesson, Centre for Qualiy Assurrance

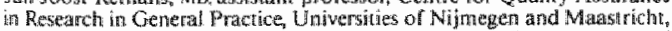

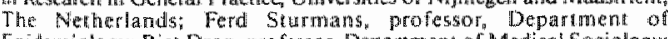

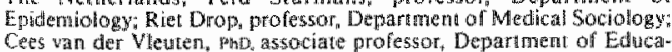

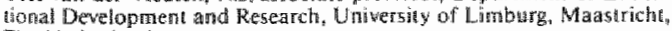
The Netherllands.

Submined: 16 May 1900; accepted 4 September 1990.

C Brivish Journas of Gemeral Pracfice, $1991.41,97-99$ docrors in real pracice performed mere actions categorized as essemal for good qualing care than they soid they would in an open ended questioniatre. Experience with the use of standwe dized pat sems who report data which can be considered reliable and valin is. however, highly limited and requires furthex testing ${ }^{4}-\bar{\delta}$

Problems arise not only in defining the meohods of assessing the performance qualisy of doctors, but exen more in defining the level of quality of care, in of her words "decting what is "com. petence", what is "good' and what is "bad". One of the most common ways to define a level of quality for performance is to set standards for actual bealth cafe. In most instances a group of experts in general practice deternines sel of standards for a particular medical problem. This procedure, however, carries the risk of setting "arnchair siandards', of standards that have no basis in actual practice." II has, tor example, been shown that a group of doctors who were first asked to assess a practical standard for a particular medicat complaint, pertormed at only $56 \%$ of their own scandard during actial consulatations. The department of general practice at the University of Limburg has undertaken a study to determine whether the aciual performance of gemeral practitioners, as assessed by standardized patients, meets predetermined standards for actural practice

\section{Method}

From anong 24 nationally accepted sets of standards of care in general practice in the Netherlands, eight medical problems were identified. which were all common in generall practice, presented a diagnostic challenge and could be presented by a standardized patient. The 24 sets of standards had been deter. mined by a consensus procedure with several stages. During the stages experts in general practice as well as general practitioners "in the field" commented on the sets of standards and cested them in practice. It was stressed that the standards should be precticable for actual practice and that they should not reflect an "acadernic view" of real practice. The standards were divided in" to three catcgories: estential actions (congidered to be recessary for good quality care), intermediate actions (not essential for good care but not ham ful either) and super thuous at fons. The aight problems which were selected for the stidy were translated into standardized roles for standardized patients to use. A pand of three general practithoners independemily ranked these poles with respect to face walidity. The fiour cases which rarked highese and on which there wat agremelat among the pand were finally chosen for this sudy. Figare I shows the most important features of the fot cases. The facts which the doctor collected about history, the results of pansical and laboratory examina tion. instructions given to the patien, treatment, and follow up were to be reported by the standardized patients and scored ac cording to the sets of standards. The number of items on each standard ranged from 26 to 36 . The standards used and their preparation process have been published in detail before." Figure 2 represents an example of one of the standards used.

Twelve standardized patients (six women and six men) were selected. Three stamidardized patients were allowated to each caste study; these were of the same sex and approximately the same 


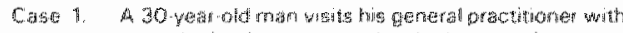

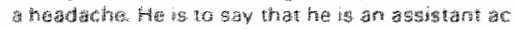

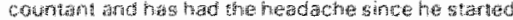

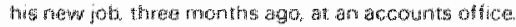

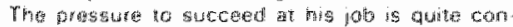

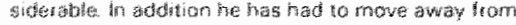

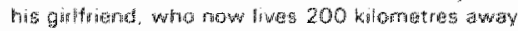

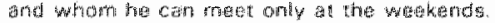

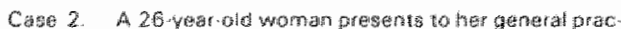

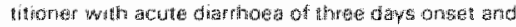
asks for somenhing of stop her complant. It the doc:

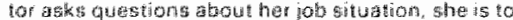

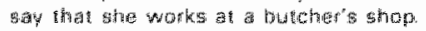

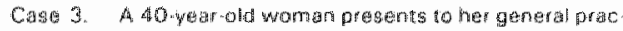
litioner with pain in one ghoulder, which she has had for low days. She is inguly oworced and has a davgher agad 75 wears. Should the thotor ask,

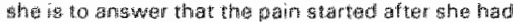
painted several door in har rew jpartment.

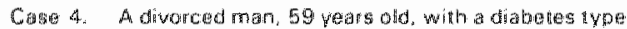
2. visitg his generb! peactituner for a new (rapeat) prascription for his ora! arytidiabotic medication.

Filgure 1. Featuras of the four ghandardized medicat complants.

\section{Hithory}

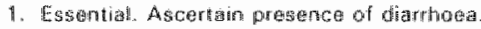

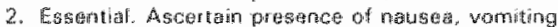

3. Enssennial. Ascertan presierice of abotominal pain

4. Lesential. Ascertain course and durtion of the complatistis.

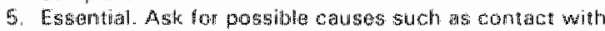
illnegrs, travel, spocial foodis atc

6. Essantial. Ask whather any other complaints are present.

7. Hotermediate. Chack medication being aken.

8. Irytermediato. Check eating habizs history.

9. intermediate. Check hor fower.

10. Essential. Ascertain stool consistercy and fraquaricy.

11. Essential. Ascortain mature of pair.

12. Esseratal. Agcertain location af pain, shifts in location.

13. Superfuous. Ask for adouthonal irtormation.

\section{Physical examination}

14. Esserital Examina atamen: inspection, percussion, auscultation, palpation.

15. Superfluous. Cospry out further examinations.

\section{Hoboritory rous a}

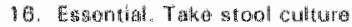

17. Sthpertuous. Cinry ou other laboratory tests.

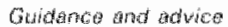

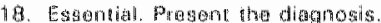

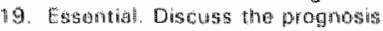

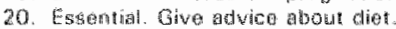

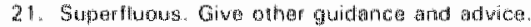

\section{Madickation}

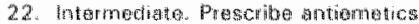

23. Irtermediato. Proscribe abgorbonts.

24. Superthons. Prescitoe oller rededication

\section{Paturn wist}

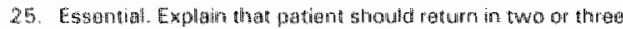

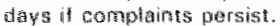

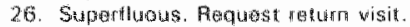

Figuro 2. The scandard of cara for the standardized potient presen-

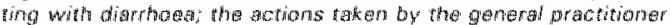
aro drydad into essentiat actions, internedisto actions and superfithous a

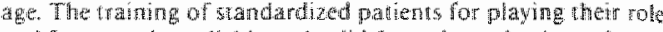

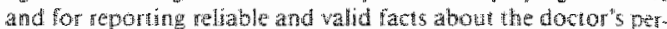

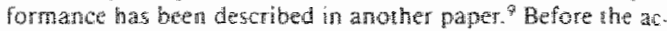

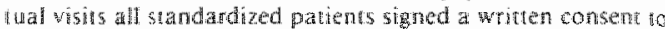

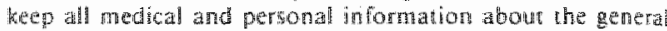
practioners in the project strety for research purposes. The doctors selected bor the study wate wisited by the sandardized patients during a four mome period staring at least 12 momth afoer they agread to paricipare.

\section{Results}

Of the 42 doctors asked 10 participate 137 (31\%) agred so be

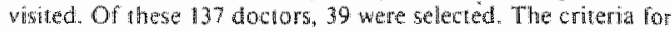
seischion have beer othined in a prewiols paper. "The persond and practice thatactistics did not differ from national charaterisitics.

"or the subcategories "essental" "intemedial and "superthous" actions, Table 1 shows the mean number of actions whish were actualy peromed by that goneral practioners for cach ol the four medcal complains. To some extert score cat be standarized as the perceniage of the potential toral mat irtum score in ach ategory of action for the sundart. Sinc: the number of superfuous actions can be potentially infitite

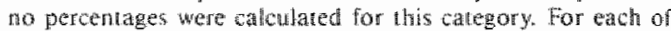
the complaits, there was a clear distinction in the adhenence to the standards betwern the esential and intermediate action. We hat aiso analysed which elements of the standards for ex ample hislory or phystol cxaminason were adheted to and whinh were ontuce, but no consistent patiern was found for the four complants. From Thbte l it can bo sen that a much smalter proponton of the maximum scores wetre oblained by the doctors for the diabertic case than for the other cases.

\section{Discussiton}

Since only $310 \%$ of doctors approached agred to participate, the restilts might reflect performance of the more competent doc. tors in the prowince of the medical school. Although the personal anci practice charactetistics of the participating docrors did not devate from national chatacteristics, we cannol exchude the possibility that the performance of non-participating doc tors would be different, possibly lower, than the reshits in this silldy.

The results showed that in actual practice doctors met only between 33-68\% of established national consensus standards for essental actons. Becatase these standards Have been deweloped for pracicat tse one would expect that doctors in actual practice would meen $90-1000^{*}$ of the standards. Since lo visits could be considtered the minimum namber to be represent tative of the ind ividual doctor ${ }^{b}$ s tastal level of per formance, the resuls ir this seudy co not allow us to look ab indivadual doc. tors, but are only applicable to the participaning doctors as a group. The fact that no comsistent pattern was fourd regarding wheh elements of the standards were adhered 10 and which were omitted, might be the resule of content specificity and of the fact that only four cases were used. An essental question in the curreat process of standard senting and especially with regard to the subsiandard scors of the participaring doctors is whether this resute reflects inadequate performance. In this whole profect 137 dontrs agreed 10 be visted ower a period of three years The fact that these doctors were not atrad of beirg audised with the very ditect method of standardized patients, shows what the had fath in their ow menhods of dealing with patienis. The conclusion from this study - wat physicians in practice per form at a lewel considerably below the standards set by their peers - is not new. However, this has never before been demonstrated in acual practice with a method as direct as the 
Thbie 1. Number of acions scared tmean. rangef tor the lour medical problems as reported by standardizen gations for consultations whth 39 gener practitioners.

\begin{tabular}{|c|c|c|c|c|}
\hline & \multicolumn{4}{|c|}{ Number of actions soored } \\
\hline & $\begin{array}{c}\text { hat } \\
10=39\end{array}$ & ngtoge & $\begin{array}{l}\text { triter } \\
\text { quartile } \\
\text { range } \\
\text { iquar 1 ro } \\
\text { guart } 31\end{array}$ & $\begin{array}{l}\text { Mean no. of } \\
\text { actions } \\
\text { scored as a } \\
\text { percantage } \\
\text { of maximum } \\
\text { no. from } \\
\text { slandard }\end{array}$ \\
\hline \multicolumn{5}{|l|}{ Hedoghe case } \\
\hline $\begin{array}{l}\text { Esseratial actions: } \\
\text { Inmaximan i3! }\end{array}$ & 89 & $5-12$ & $8-10$ & 68 \\
\hline 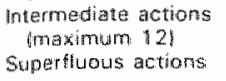 & $\begin{array}{l}3.8 \\
2.6\end{array}$ & $\begin{array}{l}0 \min 8 \\
0-7\end{array}$ & $\begin{array}{l}3-5 \\
1-4\end{array}$ & 32 \\
\hline \multicolumn{5}{|l|}{ Diarhoea case } \\
\hline $\begin{array}{l}\text { Esantial actions } \\
\text { (maximum } 15 \mathrm{j}\end{array}$ & 8.5 & $5-14$ & $7 \cdot 90$ & 57 \\
\hline $\begin{array}{l}\text { lotermediate actions } \\
\text { (maximum } 51\end{array}$ & 2.4 & $7-4$ & 13 & 48 \\
\hline Shipenfluous actions & 9.8 & $0 B$ & $0-2$ & \\
\hline \multicolumn{5}{|l|}{ Showder pain anse } \\
\hline $\begin{array}{l}\text { Essemtiat actions } \\
\text { imaximum } 191 \\
\text { finermadiate actions }\end{array}$ & 12 & $4-76$ & $10-1.4$ & 63 \\
\hline 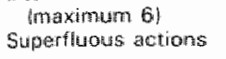 & $\begin{array}{l}1.7 \\
1.8\end{array}$ & $\begin{array}{l}0-3 \\
0-6\end{array}$ & $\begin{array}{l}1-2 \\
9-3\end{array}$ & 28 \\
\hline \multicolumn{5}{|l|}{ Diabetic case } \\
\hline $\begin{array}{l}\text { Esseritial actions } \\
\text { imaximum } 2 \text { th }\end{array}$ & 6.9 & $2-14$ & $5-8$ & 33 \\
\hline $\begin{array}{l}\text { Intermediat actions } \\
\text { (maximum } 6 \text { ) }\end{array}$ & 0.2 & 02 & $0-0$ & 3 \\
\hline Superfluous actions & 2.0 & $0-7$ & $1-3$ & \\
\hline
\end{tabular}

$n=$ number of consultations

use of standardized patienis with four visits per doctor. As already mentioned in the introduction, whether standands are external (as in this study) or internal does not seem to affeer the results obtained: doctors still performed below the standards set.

This observation of substandard performance of doctors has un the past led to actions by various professional orgatizations. These axtions were primarily directed towards mandatory attendance al continuing medical education. The underlying assump. wion of these directives is that poor performance is a reflection of ifnadequate knowledge and/or skills, which would be remediatble by additional ingtructions. Sudiles about the effects of postgraduate educanon on the behaviour of doctors in actual pracm hee often produce conflicting evidence ${ }^{\text {at }}$ It has, for instance, beten shown that additonal postgraduate education does non seen to change the practice behaviour of doctors " Recently it has also been stoow in a study of postgraduate teaching of fundascopt 10 general practinioners that there was no neacureable learning effect. although the doctors were very anthusiastic do

An alternatiye explanation to that of inadequate competenge is that physicians, when not under supervision, do not perform at the level they are capable of ${ }^{\circ}$ The willingness of all participating doctors 10 allow their act ual behaviour to be soruminz. ed, however, suggests that the results of this study do not reflect. inadequate pertormance per se. The subjective opinions of the standardized patients after their wisits stipport this vient. At the first visits in actual pardice some patients were almost enbarrassed because they fof that the doctors were really interebted in them and did their best to help them. The results of this study stress the need for more studies which unuestigate the relation. shin betwen acrual behavour and maximem competene of practising doctors.

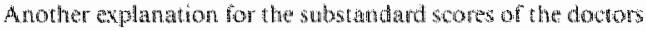
could be that the standards irwolved, now whistuding the con*

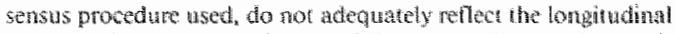

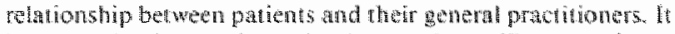
is known that in actual practice docors show efficient perform. ance and do only wher is necessary at what particulan monent.

Then large difference bet were the mean soore for the ohront diabetic case and those for the other cascs condal be becuse doclors do not ask all their questions at the lirst consuntation, but

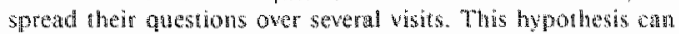
be tested by vising doctors seweral times wint the same stan* dardized patient. Our department of genenat practice is currenty involved in suct a soudy. The results of the present study seress whe need to establish standards which vake accoum of longitudinal relationshog in general practice.

The theng that doctors perfom below predetermined shandards toes not prowe that doetors are incompetent; it should at least be tested aganst the hypothest that sandard for actual care are sull not realistic.

If can be concluded that the standardized patient method is feasible and is the most ditect met hod for assessing the per formance of practising physicuans. The data fat hered with this method

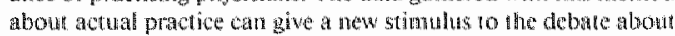
what constitutes "good" and 'bad" in acheral practice.

\section{References}

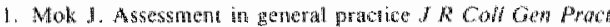
$1988 ; 38: 34$ 애 -345 .

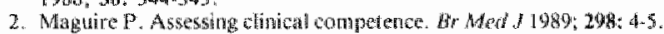

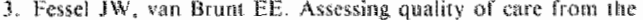
nedical record. N Ligd J Med 1972: 286: 134.138

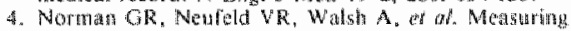

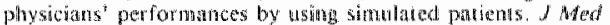
Educ 1985; 60: 925-34

9. McClure CL, Gall EP. Meredith KE, et a. Assessing ellinal

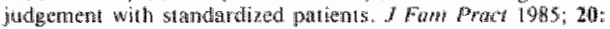
457.464 .

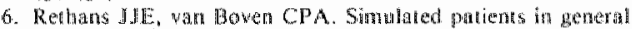

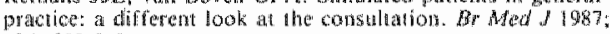
$204: 809-812$

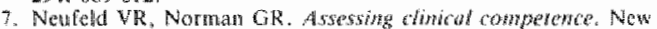
York: Springer, 1985.

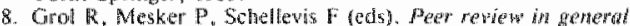
procice. Nijmegen: Lniversiny Doparthent or General Practice, 1988: 87.89; 97-98; 109-108; 117-119.

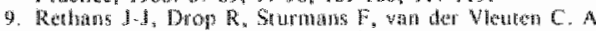

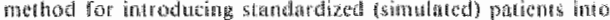

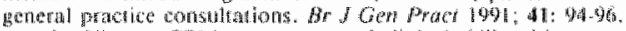

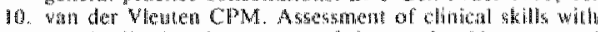

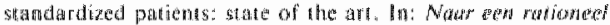

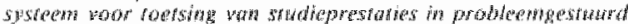

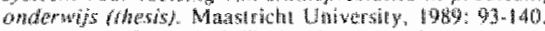

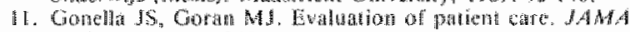
$1940+214: 2040-2043$.

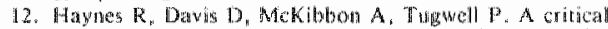

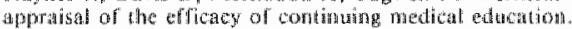
IAMA :084; 251: 61-64

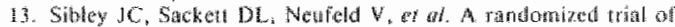

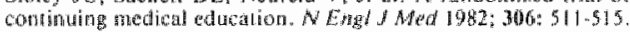

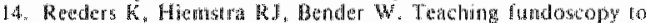

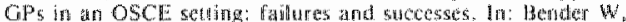

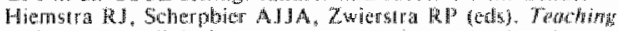

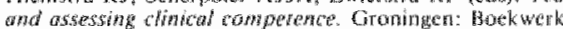
Publicatiogs, 1990

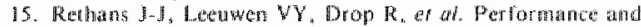

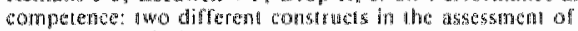

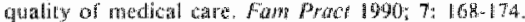

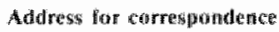

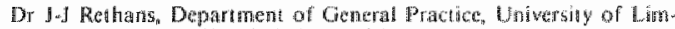

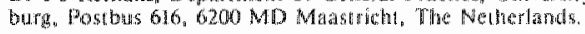


s 
Chapter 8: Does competence of physicians predict their performance?

Submitted for publication 
Does competence of physicians predict their performance? A direct comparison between an examination setting and actual practice using undetected standardized (simulated) patients

Jan-Joost Rethans, Ferd Sturmans, Riet Drop, Cees van der Vleuten, Pie Hobus

Jan-Joost Rethans, MD, Centre for Quality Assurance in Research in General Practice, Universities of Nijmegen and Maastricht, The Netherlands.

Ferd Sturmans, Professor, Department of Epidemiology, University of Limburg, Maastricht, The Netherlands.

Riet Drop, Professor, Department of Medical Sociology, University of Limburg, Maastricht, The Netherlands.

Cees van der Vleuten, $\mathrm{PhD}$, Department of Educational Development and Research, University of Limburg, Maastricht, The Netherlands.

Pie Hobus, MD, Until 1991 Department of Educational Development and Research, University of Limburg, Maastricht, The Netherlands. Since 1991, Family Physician in Wijnandsrade, The Netherlands. 


\section{Summary}

Current licensure systems for medical students/doctors are based upon the assumption that they predict actual practice. However, no hard evidence exists for this. Furthermore, it has been shown that doctors in actual practice perform below expert criteria, which led to the question whether they could perform better.

This study investigated 1) the difference and 2) the relationship between performance (what a physician actually does in his daily practice) and competence (what a physician is capable of doing).

39 Family physicians were consulted by four, incognito, standardized patients, portraying four different cases, during normal surgery hours. Later 34 of the 39 doctors participated in a controlled practice test at the Medical School, for which they were asked to act to the best of their ability. Here they were confronted with exactly the same standardized cases (different patients) as they had seen earlier in real practice. Based on the reports of the standardized patients, scores were assigned to the physicians.

Results show that mean competence score of the physicians was substantially higher than their mean performance score. The Pearson correlation between the competence score and the performance score of the participating physicians was negligable. However, when efficiency and consultation time of the consultations were taken into account, moderate to high correlations were found.

It is concluded that performance and competence should be considered as distinct constructs. Assessment of competence under examination circumstances can only have predictive value for performance in actual practice when factors such as efficiency and consultation time are taken into account. Below standard performance of physicians does not necessarily reflect a lack of competence. 


\section{Introduction}

Senior and Lloyd distinguish between competence and performance of physicians. 1,2 They define competence as "what a physician is capable of doing' and performance as 'what a physician actually does in his day-to-day practice:.

In their effort to guarantee quality of medical care, medical schools and other licensing bodies have set up examination systems to decide which students will or will not qualify as practicing physicians.

For this purpose, many countries have installed national examination bodies, whose role it is to develop, spread and apply methods of examination to assess the competence of medical students. Competence consists of knowledge, skills and attitudes. ${ }^{3}$ Assessment of competence therefore requires several measurement instruments, each representing different aspects of the construct 'competence'. The choice of a particular assessment method should be based on research on the reliability and validity of the methods. ${ }^{4}$ The use of standardized patients in exams (representing the most important aspect of medicine: a consultation with a patient) has been shown to be the most direct method, with a high reliability and high validity. $5-7$

Licensure examinations typically assess competence, whereas assessment of actual practice refers to performance. The assumption behind lisensure examinations is that competence predicts performance: passing examination predicts quality of care and performance in actual practice.

Though intuition tells that this should be the case, surprisingly no evidence exists that this assumption is true. In most studies competence and performance tend to be measured by different methods, or implicitly used concepts are not mentioned. ${ }^{8}$ An example of the latter is the use of chart audit as a competence instrument, where in fact chart audit reflects performance. The lack of evidence that competence reflects performance therefore requires the study of the relationship between competence and performance in a sound methodological manner.

The relationship between competence and performance is also important for another reason. Several studies in actual practice show that doctors perform below standards $6,9-13$, whether standards are set by experts $9-13$ or by the participating doctors themselves. ${ }^{6}$ Below standard performance appears to be a consistent finding. It has been suggested that one of the main reasons of this finding is that doctors perform below standard because they lack competence: they simply do not know how to act correctly. ${ }^{3}$

The purpose of the present study was to investigate whether indeed competence and performance as defined by Senior and Lloyd are related.8,9 For reasons of reliability and validity the method of standardized patients was chosen as an instrument. $5-7$ For the performance assessment, unrecognisable standardized patients were introduced into family physicians' normal surgery hours. Recent studies demonstrate that standardized patients may be introduced into practitioners' offices, with negligible detection 
rates $, 6,9,10$ For the competence assessment, standardized patients were used in a controlled examination setting.

It was hypothesized that, as previous studies suggest, achievement of the participating physicians in the competence situation would be higher than in the performance situation. 3,6 On the other hand, it was expected that achievements in both situations would be correlated.

\section{Subjects and Methods}

The study was divided into a performance and a competence part, which took place consecutively.

Performance part: From 24 nationally accepted and published Dutch primary care standards, eight medical problems were identified which were all common in general practice, presented a diagnostic challenge and could be presented by a standardized patient. 14 The standards describe obligatory actions (considered to be necessary) and intermediate actions (not essential but not harmful either). Any other actions are considered superfluous. The eight problems were used to construct roles for standardized patients. A panel of three family physicians independently ranked these roles with respect to face validity. The four cases which ranked highest and on which there was agreement among the panel were chosen for our study. The complaints used were 'tension headache', 'acute diarrhea', 'pain in the shoulder' and a 'checkup for a diabetic type II patient'.14 Diagram $\mathbb{1}$. represents an example of one of the standards used.

Four groups of three standardized patients (six women/six men) were selected. The three standardized patients in each group had the same gender and approximately the same age. The patients were trained to present a complaint in a standardized manner and to score history-taking, physical and laboratory examination, instructions given to the patient, treatment, and follow-up using the aforementioned standards. To assess the reliability and consistency of scoring among the standardized patients standard procedures were used, which have been carried out successfully before. 9,10 In short, the report of the standardized patient about a consultation with a physician was compared with the report by a panel about the same consultation. The obtained reliability and consistency agreement scores all ranged from 0.8 1.0 (Kappa, maximum level is 1.0). All standardized patients signed a written consent to keep all medical and personal information about the general practitioners in this project strictly for research purposes.

In January 1988 all 442 general practitioners working in the province of our university were informed about the study and asked to give their written acceptance of standardized patients into their practices for a period lasting three years and of later participation in the competence part. The doctors were not told how often or when they would be visited, nor the content of consultations, but would be informed when and by whom they had been 
wisited at the end of the project. They were asked to report every patient whom they thought they detected as a standardized patient. Four months before each of the planned visits, the standardized patients were enlisted in the practices of the participating doctors using techniques reported earlier. 15,16 The standardized patients made visits during a four months period, at least 12 months after the doctors agreed to participate.

Competence part: Five months after the visits of the standardized patients, the participating doctors were invited to the Medical School. They were installed in rooms which had been fitted out like consultation rooms of family physicians. They were then instructed, orally and in writing, to perform to the best of their abilities, free of time pressure, in their subsequent contacts with a number of standardized patients. The doctors were told that the quality (not the quantity) of their consultations was going to be assessed. The doctors were not told the number or content of cases. All consultations were audiotaped and videotaped. The same standardized patients were used and tested again for their reliability and consistency (kappa-scores ranged from .78 to .94$)$. The case histories used were the same as in the performance part, but no doctor met the same standardized patient as in the performance part.

Several variables were chosen to measure different aspects of competence and performance.

First, performance and competence scores for each doctor were calculated by counting the number of obligatory, intermediate and superfluous actions, leading to 'obligatory, intermediate and superfluous scores'. In addition, a 'total score' was calculated by summing all obligatory, intermediate and superfluous actions.

Second, since the obligatory actions (and hence their score) were regarded as the most essential part of a consultation, the ratio between the obligatory and total scores was calculated for each complaint and across the four complaints. This ratio was defined as 'efficiency score', since a high score on this variable reflects physicians with relatively more obligatory actions compared to additional actions, suggesting an efficient consultation. 10

Third, recent cognitive literature on medical problem solving suggests that the time a physician uses to solve a medical problem reflects an important aspect of expertise. 17-19 It has, for example, been shown that the longer it takes a doctor to state a diagnosis, the more likely it is that his diagnosis is wrong. 20,21 Furthermore, within a certain time span expert physicians show better and more adequate processing of relevant patient information than less experienced colleagues, suggesting that time combined with efficiency is important. ${ }^{17,22}$ In concordance with this line of reasoning, two other variables were calculated. First, for each case and across the four cases, the duration of the consultations (in minutes) was measured; the 'time score". Second, the above-mentioned 'efficiency score' was divided by this time score. This new score reflects the level of efficiency per unit of time, and was hence named 'efficiency-time score'. High efficiency-time scores 


\section{History}

1. Obligatory. Time aspects: how long have complains existed; at what times of the day; how often?

2. Obligatory. Nature of the pain.

3. Obligatory. Location of the pain, radiation, presence of prodomes; progression of complaints.

4. Obligatory. Associated phenomena (such as light phobia, nausea, fever, dental and neck complaints, etc.).

5. Obligatory. Relation with psychosocial circumstances.

6. Intermediate. What is the reaction to the pain.

7. Intermediate. Self therapy.

8. Intermediate. Intoxications (smoking, carbon monoxide).

9. Obligatory. Migraine history (familial history, progression, frequency, connection with circumstances).

10. Superfluous actions history.

\section{Physical examination}

11. Intermediate. Blood pressure reading

12. Intermediate. Examine eye sight.

13. Intermediate. Examine eye fundus.

14. Intermediate. Cervical spine.

15. Intermediate. Neurological examination.

16. Intermediate. Sinuses; percussion and pressure pain, transillumination.

17. Superfluous actions physical examination.

18. Superfluous actions Laboratory

\section{Guidance and advice}

19. Obligatory. Explain cause of complaints.

20. Obligatory. Discuss prognosis.

21. Obligatory. Explain relationship between complaints and tension.

22. A. Obligatory. In case of therapy: explain expected effect.

B. Obligatory. In case of no therapy: explain why no therapy is prescribed.

23. Intermediati:. Relaxation exercises, yoga (brochure).

24. Obligatory. Discuss connection with life style.

25. Superfluous actions guidance and advice.

\section{rherapy}

26. Intermediate. Simple analgetics.

27. Obligatory. Discuss possible background to headache.

28. Intermediate. Benzodiazepines.

29. Superfluous actions therapy.

\section{Return visit}

30. Obligatory. Indicate whether or not a retum visit is necessary, depending on possible increase of complaints and prognosis.

31. Superfluous actions return visit. 
differ from low ones in that these consultations contain relatively more obligatory actions per unit of time.

First the Wilcoxon signed rank test (paired design) was used to look for differences in the doctors' scores in the competence and performance parts. Secondly, observed correlations (Pearson product moment correlations) were calculated between doctors' scores on the two formats (disattenuated "true" correlations were not calculated, because equal content of cases were used in both formats).

\section{Results}

Of the 442 doctors asked to participate $137(31 \%)$ agreed to be visited, of whom 131 also agreed to take part in the competence part. 39 of them were selected and visited; three of them as pilot practices. For financial reasons, the main selection criterion for including a general practitioner was the distance between his/her practice and the university (less than 30 kilometres.) After all visits had taken place, 36 doctors were asked to take part in the competence part, and $34(94 \%)$ agreed to do so. Personal and practice characteristics of the participating doctors did not deviate from national data (mean years of practice-experience of these 34 doctors was 12 (range 1-28) and $15(44 \%$ ) of them were solitary working). In none of the 156 visits in actual practice was a standardized patient detected and none of the doctors was able to recall these cases, before or after the competence phase.

Table 1 shows the obligatory, intermediate, superfluous, total and time scores, as calculated from the actions performed in the performance and competence parts. The obligatory and intermediate scores have a maximum number of actions per standard, so these scores can also be calculated as percentages of a particular standard. These percentages are also shown in Table 1, under heading "\% of standard". Since the number of superfluous actions is potentially infinite, no meaningful percentages can be calculated for them. From Table 1 it follows that there was a significant difference between competence and performance for each of the variables, with competence scores being consistently higher than performance scores. The mean total score for competence across four complaints, for example, showed an increase of $49.5 \%$ compared with the same performance score. There was also a difference in the obligatory performance scores between the first three complaints and the diabetic case with respect to adherence to the standards, respectively $69,58,65 \%$ and $34 \%$.

Table 2 shows the mean efficiency scores across four complaints and for individual complaints. This table shows again that there was a significant difference between competence and performance across four cases and for three of the separate cases, but now in favour of performance. It seems that when it came to efficiency, doctors did better in actual practice than in a test. The same effect is also found in Table 3 , which shows the mean efficiency- 
Table 1. Mean obligatory, intermediate, superfluous, total and time scores (range and standard deviation) and percentages of the standard for obligatory and intermediate scores across four cases and for each individual case as reported by standardized patients visiting 34 general practitioners, for, respectively, the performance and competence settings.

Standard

Mean Range deviation \% of standard

\section{Across four cases}

Obligatory score $(\mathrm{Max}=68)$

Performance
Competence
Perliate score $(\operatorname{Max}=29)$

$37.08 * * * * * \quad 25-51$

$6.35 \quad 55$

$49.05 \quad 38-58$

$5.27 \quad 72$

Intermediate score $(\operatorname{Max}=29)$

Performance

Competence

$8.52 * * * * *$

3-14

2.59

29

12.44

9-16

2.19

42

Superfluous score

Performance

9.11 *****

2-19

Competence

20.32

10-35

3.99

Total score

Performance

$54.70 * * *$

33-83

6.68

Competence

81.82

61-102

10.05

Time score

Performance

$38^{4} 40^{\prime \prime} * * * *$

19-69

10.97

Competence

$55^{\prime} 38^{\prime \prime}$

$27-100$

$12^{\prime} 05^{\prime \prime}$

$163^{\prime \prime}$

\section{Headache Case}

Obligatory score $(\operatorname{Max}=13)$

Performance

Competence

Intermediate score $(\operatorname{Max}=12)$

Performance

Competence

Superfluous score

Performance

Competence

Total score

Performance

Conpetence

Time score

Performance

Competence

$9.02 * *$

10.64

$4.08 * * *$

5.58

$2.70 * * *$

5.23

15.82

21.47

$113^{\prime \prime \prime} * * * * *$

$17^{\prime} 07^{\prime \prime}$
5-12

$7-13$

0-7

$3-9$

0-7

$1-13$

$11-2 \mid$

13-29

4-19

7-28
1.62

1.25

69

82

1.65

1.63

34

47
1.91

2.73

2.79

4.17

$3^{\prime} 45^{\prime \prime}$

$5^{\prime} 01^{\prime \prime}$ 
Mean

Range

Standard

deviation $\%$ of standard

Diarrhea Case

obligatory score (Max $=15)$ Performance

Competence

Intermediate score $(\mathrm{Max}=5)$

Performance

Competence

Superfluous score Performance

Competence

Total score

Performance

Competence

Tine score

Performance

Competence

$\begin{array}{lccc}8.67 * \cdots & 5-14 & 2.4 & 58 \\ 12.08 & 9-15 & 1.5 & 81 \\ 2.50 * & 1-4 & 1.05 & 50 \\ 2.94 & 1-5 & 0.91 & 59 \\ 1.88 * & 0-8 & 1.88 & \\ 3.17 & 0-8 & 1.78 & \\ 13.05 * \cdots * & 6-23 & 4.03 & \\ 18.20 & 13-23 & 2.38 & \\ 6^{\prime 4} 47^{* * *} * & 3-12 & 2^{\prime} 36^{\prime \prime} & \\ 9^{\prime} 42^{*} & 4-18 & 3^{\prime} 32^{\prime \prime} & \end{array}$

Shoulder pain Case

Obligatory score $(\mathrm{Max}=19)$

Performance

Competence

Intermediate score $(\mathrm{Max}=6)$

Performance

Competence

Superfluous score

Pertormance

Competence

Total score

Performance

Competence

Time score

Performance

Competence

$12.29 * *$

5-16

3.13

65

14.23

9-17

1.89

75

$1.76 *$

$0-3$

0.92

29

2.67

$1-5$

1.17

44

$\frac{2.50 * * * *}{5.08}$

0-7

1.60

5.08

$1-9$

1.91

$16.55 * * * * *$

6-24

4.26

22.00

14-28

3.42

$8^{\circ} 00^{11 * * * * *}$

3-13

$2^{\prime} 56^{\prime \prime}$

$12^{\prime \prime} 08^{\prime \prime}$

5-26

4'28"

Diabetic Case

Obligatory score $(\operatorname{Max}=21)$

Performance

Competence

Intermediate score $(\mathrm{Max}=6)$

Performance

Competence

Superfluous score

Performance

Competence

$7.08 *$ **3 水将

2- 14

2.81

34

12.08

6-19

3.03

58

$0.17 * * * *$

$0-7$

0.45

0

1.23

$0-3$

0.78

21

Total score

Performance

Competence

Time score

Performance

Competence

$2.00 * * *$

0-7

6.82

0-24

1.79

5.72

$9.26 * * * *$

$2-18$

4.04
8-45

20.14

$\begin{array}{lll}12^{\prime} 19^{* * *} * & 4-36 & 6^{\prime} 55^{\prime \prime} \\ 16^{\prime} 40^{\prime \prime} & 7-28 & 5^{\prime \prime} 53^{\prime \prime}\end{array}$

$\begin{array}{lll}12^{\prime} 19^{* * * *} & 4-36 & 6^{\prime} 55^{\prime \prime} \\ 16^{\prime} 40^{\prime \prime} & 7-28 & 5^{\prime \prime} 53^{\prime \prime}\end{array}$

7.87

$* * * 0 * 0.0000$

*** $p<0.0005 ; * \mathrm{p}<0.005 ; * \mathrm{p}<0.05$; Wilcoxon signed rank test (paired design) 
Twble 2. Mean efficiency score (range and standard deviation) across four cases and for each individual case as reported by standardized patients wisiting 34 general practitioners, for, respectively, the performance and competence settings.

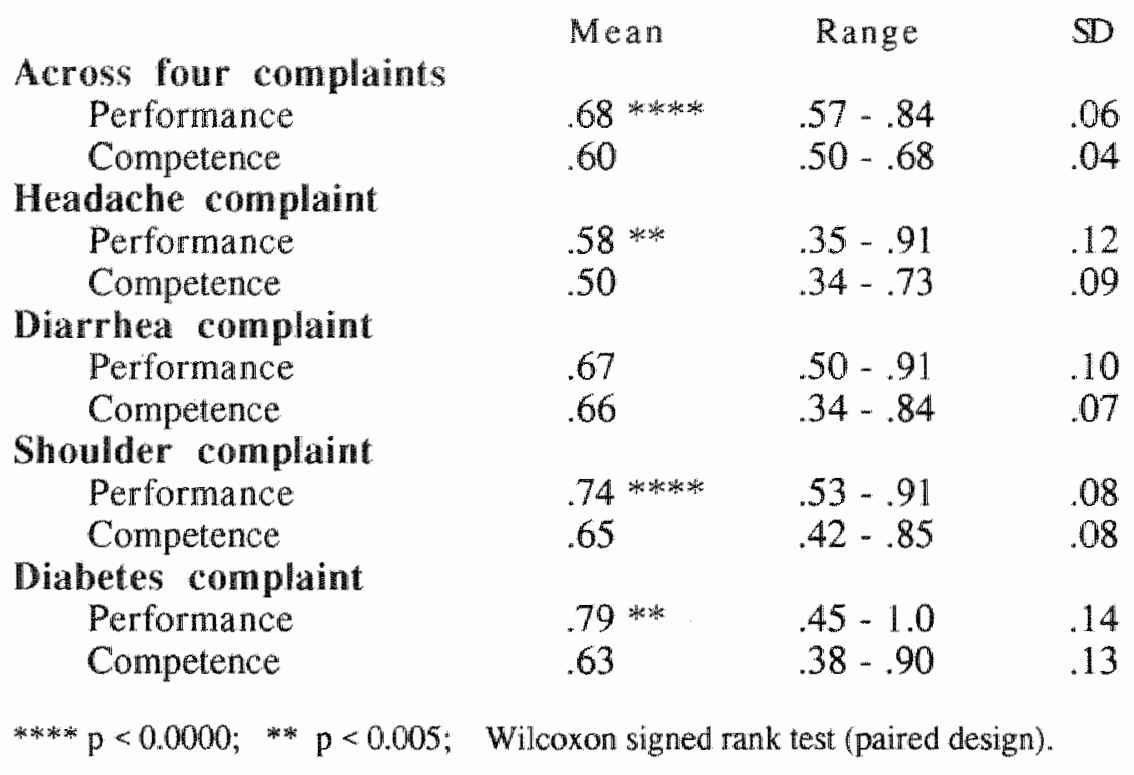

Table 3. Mean efficiency-time score (range and standard deviation) across four cases and for each individual cases as reporled by standardized patients visiting 34 general. practitioners, for, respectively, the performance and competence settings.

\begin{tabular}{|c|c|c|c|}
\hline \multirow{2}{*}{\multicolumn{4}{|c|}{ Across four complaints }} \\
\hline & & & \\
\hline Competence & .011 & $.005-.023$ & \\
\hline \multicolumn{4}{|c|}{ Headache complaint } \\
\hline Performance & $.058 * * * * *$ & $.027-.211$ & \\
\hline Competence & .034 & $.092-.104$ & .0 \\
\hline \multicolumn{4}{|c|}{ Diarrhea complaint } \\
\hline Performance & $.121 * * * *$ & $.050-.277$ & \\
\hline Competence & .080 & $.036-.176$ & .0 \\
\hline \multicolumn{4}{|c|}{ Shoulder complaint } \\
\hline Performance & $.108 * * ; *$ & $.053-.277$ & \\
\hline Competence & .060 & $.026-.150$ & \\
\hline \multicolumn{4}{|c|}{ Diabetes complaint } \\
\hline Performance & $.084 * * * *$ & $.020-.250$ & .0 \\
\hline Competence & .044 & $.016-.093$ & \\
\hline
\end{tabular}

$* * * *<<0.0000 ; * * * \quad p<0.0005 ; \quad$ Wilcoxon signed rank test (paired design). 
time scores for the competence and performance settings. Across four cases, for example, the mean efficiency-time score in the performance setting was $65 \%$ higher than its equivalent competence score.

Table 4 shows the correlations between competence and performance for each of the variables used. The table shows several surprising outcomes. The correlations of .00 and -.04 for, respectively, the obligatory score and the total score across four

complaints, suggests a disappointingly low (virtually no) correlation between competence and performance. The same conclusion can be drawn for the efficiency score. Surprisingly, this score shows correlations which do not deviate much from the intermediate and superfluous scores. In contrast, the time and efficiency-time scores across four cases seem to indicate a more substantial correlation between competence and performance. The individual cases in Table 4 show the same interesting pattern: very low correlations for the obligatory and total scores, but high values for the time and efficiencytime scores.

However, as in Table 1, the diabetic case of Table 4 again shows a pattern that is different from the other complaints, with many negative values, in contrast to the other cases. For this reason we also calculated what the correlation between competence and performance would be without the diabetic case. The results are also included in Table 4 . These values (without diabetes) show again that the obligatory and total scores yield a low correlation. The efficiency-time score, however, yielded high correlations, up to .77 , between competence and performance.

Table 4. Pearson product moment correlation between competence and performance for the obligatory, intermediate, superfluous, total, time, efficiency and efficiency-time scores across four cases, across three cases and for each individual case with 34 physicians.

Across
four
complaints

\begin{tabular}{|c|c|c|c|c|c|c|}
\hline Obligatory score & .00 & .07 & -.11 & .20 & .15 & .21 \\
\hline Intermediate score & .26 & .33 & .25 & .07 & -.04 & .32 \\
\hline Superfluous score & -.05 & .24 & .25 & .10 & .25 & .25 \\
\hline Total score & -.04 & .23 & .24 & .07 & -.07 & .29 \\
\hline Time score & $.49 *$ & $.48 * * *$ & 67 & $.47 *$ & .15 & $.61 \leqslant$ \\
\hline Efficiency score & -.08 & $.34 *$ & .32 & .28 & -.30 & $.35 *$ \\
\hline Efficiency-time score & $.45 * *$ & $.72 * * *$ & $59 *$ & $.62 *$ & .00 & 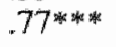 \\
\hline
\end{tabular}




\section{Discussion}

This is the first time a study has compared actual performance of doctors in their practices with data collected in an examination setting, both assessed with the direct method of standardized patients. Clearly, the study has some limitations.

First, competence has been reduced to its medical-technical aspect, which means that physicians' attitudes and knowledge were not studied.

Second, this study used only four cases per doctor, which is too few to allow generalization beyond the specific sample of cases used. ${ }^{7}$

Third, $31 \%$ of invited doctors responded positively. The question arises whether these doctors represent a selected group with higher standards of care than non-participants, although personal and practice characteristics of the participating doctors did not deviate from national data. If it is assumed that the doctors under study are the better ones, then it remains unclear whether the competence-performance difference of the non-participants is likely to have been larger or smaller.

Fourth, there is the validity question of the standards: would different standards have produced different results? As was explained, the standards were developed for use in actual practice. As the designers were well aware of the risk of setting expert criteria, the construction procedure consisted of several rounds of comments including a pilot phase in practice. 16 Therefore, at the start of this study there was no reason to doubt the validity of these standards.

With these limitations in mind, several important results emerge from this study.

First, from the large, significant differences between the competence and performance scores for all the variables used, it can be concluded that there is a substantial difference between competence and performance of physicians.

Second, the direction of the differences in scores between the two formats is very interesting and challenging. The use of only quantitative data (obligatory, intermediate, superfluous and total score) shows that doctors did more in the competence than in the performance situation. However, using qualitative data (efficiency and efficiency-time score), it was in actual practice that doctors did better.

Third, a major finding of this study is that it appears to be crucial to choose the correct variable to measure the correlation between competence and performance. This is shown by the differences in level between, on the one hand the obligatory, total and effiency correlations and, on the other hand the time and efficiency-time correlations. It is difficult to explain why the level of the efficiency score correlation is not higher than those of the obligatory, intermediate and superfluous scores. Perhaps this reflects that scores, without the time-factor, are bad predictors of performance. The high time correlation seems to indicate that time is also an important factor with respect to 
expertise. As regards the differences between the first three cases and the diabetic case, the participating doctors were asked about the reasons for this difference. They responded that the diabetic case, in contrast with the others, reflected a chronic disease, for which in general practice several consultations would normally be used in order to gather all necessary patient data. The type of problems, therefore, remains an important issue in real practice as well.

We believe the implications of the study are in two areas, viz. the examination-setting or licensing area and the area of setting standards for the quality of care.

First, since the doctor-patient consultation is the final purpose of medical education, assessment of students or physicians' capacities in this contact is crucial. This study shows that, if qualitative data are used in combination with the time that examinees need to perform a test, competence will indeed be predictive of performance. However, examinations are generally restricted to quantitative data. This study shows that these data are poor predictors of performance, casting serious doubts about the validity of current examination systems. It seems crucial to make use of proper variables in order to be able to predict performance from competence situations. Second, with respect to standards of care it can be concluded that this study shows that doctors perform below standard in actual practice, but that they are able to perform significantly better when asked to do so. It can be concluded that the physicians are more competent than their performance in practice shows. On the other hand in the competence test they also perform below the standards. Taking into account other experiments with internal or external standards, it might be asked whether the procedures of setting standards are valid.6,9-13 Even when these procedures contain pilot-practice phases and even when doctors are asked to formulate standards themselves, results show that doctors perform below standards. The finding in this study that the participating doctors had good reasons not to adhere to the diabetic standard, shows that one has to be cautious about interpreting the scores of physicians on standards. The finding that doctors act more efficiently in real practice than in a test, also means that one should be cautious in simply concluding that doctors just have to do better, in relation to the standards, than in real practice. It has been shown before, also with standardized patients, that doctors in actual practice show efficient performance. 10

The participating physicians were left free of time pressure in the competence part of our study. The reason for doing so was that we wanted to investigate whether doctors would indeed be able to perform more actions in the competence situation than in actual practice. It would be interesting now to investigate what would happen if doctors were to perceive time pressure in the competence setting as well.

It seems necessary to reconsider carefully the procedures for setting standards. It might be advisable to start with an assessment of actual practice in a valid way (by observing how doctors really perform). In this process standardized patients can play an important role. Since the standardized 
patients were highly satisfied in nearly all consultations, even though the doctors varied in their level of scores, it is suggested that the number of actions performed alone can never be the sole base for assessing a physician's competence.

From this study it can be concluded that there is a difference between competence and performance of physicians. Taking qualitative data into account, competence is a predictor of performance. This should be taken into consideration in examination systems and in decisions about the way doctors have to be assessed for (re)certification reasons.

Quality of care in actual practice should not be assessed on the basis of standards alone. Other aspects of actual practice, such as patient satisfaction, consultation time and outcome of consults, should also be taken into consideration.

\section{Acknowledgments}

We are grateful to the 39 participating familly physicians in Limburg, to Caroliene Janssens, Marike Laning, Marijke Verdonk, Bea de Grootte, Martha Lucassen, Juul Kerbusch, Peter Kramer, Pieter Ramler, Pierre Bastings, Leon Heuts, Wil Macco, Trudie Seegers, the sickfunds VGZ in Maastricht and LIASS in Heerlen and Sittard, and Robin Hull of the General Practitioners Writers Association for their efforts in this study.

\section{References}

1. Senior JR. Towards the measurement of competence of medicine. Philadelphia: National Board of Medical Examiners 1976.

2. Lloyd IS. Definitions of competence in specialties of medicine. Chicago: American Board of Medical specialties 1979.

3. Neufeld VR, Norman GR. Assessing clinical competence. Springer Publishing Company, New York, 1985.

4. Maguire P. Assessing clinical competence. Br Med 11989;298:4-5.

5. Stillman PL, Swanson DB. Ensuring the clinical competence of medical graduates through standardized patients. Arch Intern Med 1987; 147: 1049-52.

6. Norman GR, Neufeld VR, Walsh A, Woodward CA, McConvey G. Measuring physicians' performances by using simulated patients. J Med Educ 1985; 60: 925-34.

7. Vleuten CPM van der, Swanson DB. Assesment of clinical skills with standardized patients: state of the art. Teaching and Learning in Medicine 1990; 2: 58-76.

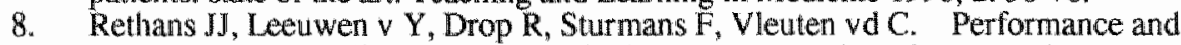
competence: two different constructs in the assessment of quality of medical care. Fam Pract 1990; 7: 168-74.

9. McClure CL, Gall EP, Meredith KE, Gooden MA, Boyer JT. Assessing clinical judgment with standardised patients. J Fam Prac 1985; 20: 457-64.

10. Rethans JJE, Van Boven CPA. Simulated patients in general practice: a different look at the consultation. Br Med J 1987; 294: 809-12.

11. Norman GR, Tugwell P, Feightner JW. A comparison of resident performance on real and simulated patients. J Med Educ 1982; 57: 708-15.

12. Lomas J, Anderson GM, Domnick-Pierre K, et al. Do practice guidelines guide practice? N Engl J Med 1989; 321:1306-11. 
13. Grol R. Kwaliteitsbewaking in de huisartsgeneeskunde. Krips Repro Meppel, 1986. Thesis. (Summary in english)

14. Grol R, Mesker P, Schellevis F (eds.). Peer review in general practice. Nimmegen University Department of General Practice, 1988: 87-9; 97-8; 107-9; 117-9.

15. Rethans JJ, Drop R, Sturmans F, Vleuten vd C. A method for introducing standardized (simulated) patients into general practice consultations? Br I Gen Pract 1991; 41:94-6.

16. Rethans JJ, Sturmans F, Drop R, Vleuten vd C. Assessment of the performance of general practitioners by the use of standardized (simulated) patients. Br J Gen Pract. $1991 ; 41: 97-99$.

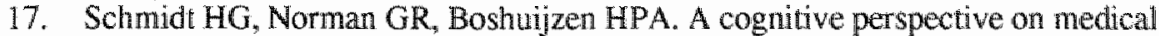
expertise: Theory and implications. Acad Med 1990;65:611-21.

18. Schmid HG, Boshuijzen HPA. Hobus PPM. Transitory stages in the development of medical expertise: the intermediate effect in clinical case representation studies. In Proc. 10th Conf Cognitive Science Soc. Hillsdale, New Jersey: Erlbaum; 1988: 139-45.

19. Norman G, Allery 1, Berkson L et al. Research in the psychology of clinical reasoning: implications for assessment. In: Jolly B (ed). New directions in the assessment of clinical competence. Proc Cambridge Conf IV, Madingley Hall, Cambridge. In press.

20. Kundel HL, Nodine CF, Carmody D. Visual scanning, pattern recognition and decision making in pulmonary nodule detection. Invest. Radiol. 1987; 13: 175-81.

21. Norman GR, Brooks LR, Allen SW, Rosenthal D. The development of expertise in dermatology. Arch Dermatol 1989; 125: 175-81.

22. Hobus PPM, Schmidt HG, Boshuizen HPA. Patel VC. Contextual factors in the activation of first hypotheses: expert-novice differences. Med Educ 1987; $21: 471-76$. 

Chapter 9: Conclusions and recommendations for research 
" 
In the introductory chapter it was stated that the papers in this book had two main aims.

The first of these was to investigate whether it was possible for a set of standardized (or simulated) patients to visit general practitioners during normal surgery hours without being detected. The second aim was to investigate 1) the difference and 2) the relationship between the competence and performance of general practitioners, with performance defined as "what a doctor does in his day-to-day practice" and competence as "what a doctor is capable of doing". 1,2

In this chapter the main conclusions of the projects will be discussed. Some advice for further research in the field of standardized patients and of competence and performance will be given.

\section{The methodology of standardized patients.}

In the first of the two real practice experiments described here, only two out of 48 visits by standardized patients have been detected, and none in the 156 consultations of the second project. Two patients in the second project who were reported to be standardized patients tumed out to be genuine. Together with the results of the assessment of the consistency of the standardized patients (kappa 0.78 to 1.0 ), these findings show the feasibility of this method in a health care system, even where doctors have fixed patient lists.

The main reason for this success probably lies in the detailed procedures used for both the training and for the actual visits by the standardized patients. In discussions with other researchers involved in the training of standardized patients at the Fourth Ottawa Conference on Assessing Clinical Competence (July 1990), it became obvious that the length of the training (role playing and reliability training) of our patients (14 hours) was rather long. 3 The other researchers mentioned having used about half the training time we used in our projects. However, they did not have experience with the use of standardized patients in real practice and it was for this real practice purpose that we in our project were more afraid of using too short a period of training than one which was too long. It is difficult to estimate whether the procedures used in our projects were too detailed; in the words of one of the participating physicians: "How much of your preparation has been superfluous?". Some anecdotal reports by our patients seemed to indicate that nothing was superfluous when it came to preventing the detection of standardized patients. For example, as explained in chapter 6 , the selection of home addresses for the standardized patients was an important feature in the preparation phase for the actual visits. If it was impossible to find a credible address by searching in the computer of another doctor with a practice in the same city, and if it was also impossible to "borrow" adresses from friends or relatives of the researchers, a risk was taken by selecting a non-existing house number in a known street or by 
selecting a random number in an apartmentbuilding. One standardized patient, who was supposed to live at such a random number in an apartmentbuilding, reported that at the start of the consultation the general practitioner he was going to visit showed him a medical file with the same surname and address as the one he had just been given, and asked: "Is she your sister?". So, accidentally we had given the standardized patient both the same name and the same address as an genuine patient. In this case it was enough for the standardized patient to present the medical assurance card to convince the doctor that the (real) address on the existing medical file was likely to be false.

Although during a large number of visits such details as "being familiar with the neighbourhood of the doctors visited' was not a point which was discussed during the actual contacts, these details were crucial during some other visits. They also helped the patients to feel secure during the visits.

In general, it can therefore be said that as a means of preventing detection of any standardized patient, nothing in the preparation phases was superfluous. The question is however whether it is necessary that not a single case is detected. To answer this question a series of experiments would be necessary.

At the start of these projects the majority of general practitioners in the region of the university were unfamiliar with the method of standardized patients, since it had never been introduced in actual practice. Given this fact, we estimate that the figure of $31 \%$ of invited doctors who agreed to participate in this project is a high one.

Although the personal characteristics of the participating doctors and their practices did not deviate from the national characteristics, we cannot exclude the possibility that the participating doctors represent the "cream" of all doctors.

Several months after the visits had taken place we informed the participating doctors about the nature and content of the visits. All of them reacted positively to the visits, that is to say that they had no objection to the fact that details of the medical part of their contacts with the patients were to be reported. We were told that the guarantee of anonymity given to the participating doctors helped a great deal in creating this positive feeling. We think that in future, with the results of the present book at hand, the percentage of doctors who will be willing to participate will increase.

What are the prospects for the method of standardized patients in real practice after the experiments described here? In other words, will the method be used more often, and for what purposes?

Firstly, the experiments have led to a discussion about the way in which audit will have to be organized in practice. This discussion was reflected by several letters to medical journals and other media.4-8 The main issue in the debate about the prospects for this method seems to be whether the method should be used only if doctors give prior permission for the introduction of standardized patients. Although this seems a reasonable demand from the 
point of view of the doctors and their organizations, it may well lead to a different opinion when viewed from the perspective of the patients. In fact, the use of standardized patients in real practice consitutes audit by consumer and we tend to consider that the consumer's opinion is more important than that of the doctors.

Secondly, our data collected with the standardized patient method is restricted to the medical technical findings during the consultations. However, in a non-systematic way we have also reported, in chapter seven, on the opinions of the standardized patients as regards the caring qualities of

the participating doctors. Research with standardized patients for educational and assessment purposes has shown that standardized patients are also capable of reporting in a consistent manner on the social and technical skills of medical students. ${ }^{9}$ It would therefore be worthwhile to extend research in real practice with standardized patients, reporting also on the social and attitudinal skills of physicians.

Thirdly, with regard to research on inter-doctor variation the method of standardized patients will be potentially the best method, since by using standardized patients researchers will be able to present the same stimulus (the standardized patients) to a set of different doctors and be able to compare the difference in performance between doctors. Of course, also in the future, whether the method of standardized patients is the best one to use will still depend on what researchers are looking for in actual practice. For very detailed, for example semantic, analysis of consultations, other methods such as videotaping or audiotaping may be even better.

However, with regard to the assessment of the performance of doctors in their day-to-day care of patients, the method of standardized patients is the best one to use. It is also our experience that this type of research is furthermore very enjoyable for researchers, for standardized patients and for the participating doctors.

\section{Competence and performance.}

Several interesting results have emerged from the research conducted.

Firstly, the findings show that all the doctors participating performed substantial more actions in the competence setting than in the pertormance setting. This result was consistent for all categories used: obligatory, intermediate and superfluous actions. It is unequivocally clear that doctors perform more of the actions mentioned in the standards in a test selting than in actual practice, something which was a matter of debate before our project started.

Secondly, an interesting finding was also that when it comes to efficiency (with efficiency defined as the ratio between the obligatory actions and the number of total actions per consultation), doctors performed substantially better in real practice than in the test setting. This finding suggests that 
doctors may have very good reason for carrying out, or not carrying out, certain actions in practice. For example, this was well demonstrated by the reaction of the participating doctors to their performance in the diabetic case. When the doctors were asked for comments on their low performance (according to the standard used), they argued that when dealing with chronic patients (and complaints) they did not intend to perform all the actions during a single visit. They stated, that working as a general practitioner means that patients are being seen in a longitudinal manner, i.e. during consecutive visits thoughout the years, giving ample opportunities gather data. They argued that the diabetic standard did not take account of this real life fact.

Future research in this area may be focussed on the reasons for the differences found and on the reasons why certain actions were or were not performed. One way to conduct such a study is to introduce standardized patients into general practitioners' practices and to inform the doctors immediately after the consultation, that they have been visited. Then, with the report of the standardized patient at hand, the researchers could ask the doctors, for each of the reported items, for the reason for performing or not performing medical actions. The design of such a study could also be used to study the effects of feedback on real performance of physicians. Feedback in real practice by means of a standardized patient immediately after a consultation is probably very effective, since experience with it for educational purposes is well described. 10,11

Thirdly, a major finding of this study is that it appears to be crucial to choose the correct variable to assess the association between competence and performance. This is shown by the difference in level of the competenceperformance correlations, between on the one hand the obligatory, total and efficiency (competence-performance) correlations and, on the other hand the time and efficiency-time (competence-performance) correlations.

It is difficult to explain why the efficiency score (competence-performance) correlation is not higher than the competence-performance correlations for the obligatory, intermediate and superfluous scores. It suggests that scores without the factor time are bad predictors of performance. The high correlation on the variable "time" seems to indicate that time is an important variable with respect to expertise.

A recent cognitive theory as to the development of medical expertise may clarify this finding. ${ }^{12}$ In this paper a new theory for the development of medical expertise is presented: "Contrary to existing views, this theory assumes that expertise is not so much a matter of superior reasoning skills or in-depth knowledge of pathofysiological states as it is based on cognitive structures that describe the features of prototypical or even actual patients. These cognitive structures, referred to as "illness scripts", contain relatively little knowledge about pathofysiological causes of symptoms and complaints but a wealth of clinically relevant information about disease, its consequences, and the context under which illness develops". 
The authors describe their theory as a stage theory: during their medical education students/doctors pass through several hiearchical stages, each representing a phase in the ascent to expertise.

The first stage is one of forming so-called "propositional or conceptual networks". When confronted with medical problems and solving them students search for events, concepts or objects which can be related to each other and they then try to make causal networks out of them.

The second stage starts with their being confronted with real patients. Having met the same case or problem before (or cases with same features to those already met), shortcuts will emerge in the student's diagnostic process. His knowledge-in-use will reorganize itself (by shortcuts) so that accessibility and efficient use are assured.

The third stage is one of the emergence of "illness scripts". This may be simplified by imaging that, simultaneously with a further compilation of knowledge (stage 1-2), students begin to pay attention to contextual factors under which diseases emerge. Instead of causal processes (stage 1-2), the different features that characterize the clinical appearance of a disease become more important. Problem solving in routine cases thus becomes a process of "script search, script selection and script verification".

The fourth and final stage is called "storing patient encounters as instance scripts". In its essence this stage is characterized by the finding that experienced doctors solve cases by recognizing a new presentation simply on the basis of its similarity to one previously encountered. The theory would predict that when dealing with medical problems which they have not seen before, doctors switch to stages which are lower in the hierarchical system presented. This means that physicians dealing with a more or less unfamiliar problem try to solve it with knowledge/experience at a more basic level and start reasoning towards the solution of a problem on this basis.

In their arguments for presenting this theory, the authors repeatedly discuss the influence of time on the process of clinical reasoning. They argue that the amount of time a physician needs to solve a medical problem reflects an important aspect or results of expertise, in that less time needed reflects better expertise. 13-15 More experienced physicians make more use of "instance scripts", and this takes less time then problem solving.

Furthermore, within a certain time-span, expert physicians compared to less experienced colleagues show better and more adequate processing of relevant patient information, suggesting that time combined with efficiency is important. 13,16

Our data does not allow accurate measurement of differences between experienced and less experienced doctors, since the range of years of experience of our doctors is too small, but the issue of time should certainly be taken up in new studies. In the competence part of our study we have stressed that the doctors should take their time, but we did not say "please take as much time as you can". Of course the effects of this remark to the doctors can be regarded as a bias: all doctors used more time than in the 
practice setting and they thus performed more actions. As we have said earlier in this discussion, a major aim of the study was to see if doctors could perform better. We therefore intended to give the doctors as much time as they desired. Suppose we had put the doctors under stress in the competence part by saying they would be rewarded by taking a very short time, or by sayng that they would be punished for using too long a time, or by saying that they were supposed to treat 40 patients in two hours. If the result of such a study had been that doctors performed fewer actions in the competence part than in the performance part for all variables used, the design could be criticized for not giving the doctors enough leeway to spread their talents.

With the results of the current study at hand it is advisable to conduct the same study as we did, but now with two random groups: one with and one without "time-stress" in the competence part. In this way the same variables (and their competence performance correlations) as we used could be compared under two different conditions.

On the basis of our results other experiments with the factor time might also be worthwhile. We would like to suggest studying what will happen with the results of medical students in examination settings when these results are corrected for the amount of time students take to solve the problem. So far, this has not been usual in current examination systems. ${ }^{17}$ When two students score the same number of points on the Maastricht Progress Test and one of them took four hours and the other only one hour to solve the problems, the results are the same: the same number of points. If tests are supposed to differentiate between levels of medical expertise, then possibly time could be taken into consideration in the outcome.

There is one important remark, or rather restriction, to be made with regard to the conclusions about the factor time in our study. We have not measured time per unit or part of a consultation. For example, the stagetheory deals with time from a cognitive perspective, that is to say that time is measured only with respect to stating a diagnosis or to solving a problem. Besides the cognitive part of a consultation our measurement of time also contains other parts, such as time to shake hands, time to show the patient the way to the pharmacy, time to explain the diagnosis, time to help (un)dress the patient, time to fill in a lab form etc. This implicates that although time seems to be an important factor, more precise measurement of time (for example per unit of consulatation) may well show that in some respects time may be less important. This shows that future studies with respect to the impact or result of time need to specify which component of the consultation the time is needed for. 


\section{Standards of care}

The studies reported in this book show that doctors in real practice perform below standards of care. The mean numbers of actions performed by the doctors in real practice were, for the obligatory category according to the standards, $69,58,65$ and $34 \%$ for respectively the headache, diarrhoea, shoulder and diabetes cases.

Since below-standard performance has been demonstrated before, this may not be seen as an innovative finding. It is therefore more interesting to compare these performance values with their equivalent competence values, which are $82,81,75$ and $58 \%$ respectively. These values show without doubt that doctors are able to perform better in a test situation than in real practice.

Things get complicated, however, with the finding that with regard to efficiency doctors do better in the real practice situation than in the test situation.

What does this result mean for the current policy of standard setting in primary care? We think that the results show that one has to be careful when drawing conclusions about doctors' performance, if these conclusions are onlly based on scores on standards, reflecting competence assessments. The results also show that one has to take account of what actually happens in general practice. We have asked the participating doctors, in a not-systematic manner, about their explanation for the large difference in performance scores between the diabetic case and the other three cases and we have discussed this already (section "competence and performance"). Their main criticism was that the diabetic standard did not take into account the longitudinal aspects of consultations in general practice: patients will be seen at consecutive points in their life and during illness. This longitudinal aspect of general practice is not only very important in chronic diseases, it is also important in more or less acute diseases. The knowledge a specific general physician has about a specific patient with regard to such factors as age, sex, family, use of medication, number of visits per year etc (factors often known as "contextual factors") has been shown to be very important for the diagnostic aspect of consultations. 18 We think that the finding in our study that doctors show more efficient behaviour in real practice (and in less time) than in the competence part is a reflection of this phenomenon. If standards in general practice are to be effective with regard to an increase in the quality of general practice (as is the target of the policy makers who produce standards), then standards should at least cover both the longitudinal and the contextual aspects of general practitioners' work. However hardly any of the current standards seems to cover these issues.

Does this mean that standards are useless, a waste of time? No, we do not think so. They are certainly valuable, but until now mainly for educational reasons. The process of setting standards in general practice is in its infancy, and the Dutch College of General Practitioners (Nederlands Huisartsen 
Genootschap) is well ahead of other national colleges with respect to the process of standard setting. The Dutch College should however be aware of the fact that the process by which standards are currently being made is likely to be a waste of time and money if general practice concepts are not taken into account more explicitly.

It is helpful to know that the main reason for starting the current standards policy of the Dutch College of General Practitioners was not a perceived need to increase quality in general practice, but more fear of governmental intervention (for budgetary reasons) in the autonomy of general practitioners. The College thought it was better to start a quality initiative itself than to be forced into accepting governmental supervision on this issue. Nevertheless this initiative may well lead to good results with regard to improving quality, if the standards are accompanied with quality assurance programmes. Standard setting as a target in itself will never succeed in raising the quality in general practice. Standard setting committees should be aware of the phenomenon of "arm-chair standards", a term used to describe the danger that committees, in their desire to produce consensus standards, design standards which do not have much to do with day-to-day practice. 19

Our results show that although doctors did not perform according to the standards in the performance part, they were able to act substantially better in this respect in the competence part, but still not fully at the level of the standards. This finding implies at least three hypotheses.

Firstly, the doctors are not able to give good quality of care, since they fail to reach the standards even in the competence part.

Secondly, the standards were not realistic, meaning that they did not take account of the actual capacities of doctors in the performance part as well as in the competence part.

The third hypothesis is a mixture of the first two: doctors do not perform well enough and the standards will have to take more account of day-to-day practice. We tend to think that the third hypothesis will be the one most likely to be true.

We therefore think that more research is necessary with regard to standards in general practice. We would like to suggest conducting more studies to find out what happens in actual consultations in general practice. This can be done either by standardized patients or by other methods which are able to clarify the real practice of doctors, such as videotapes of consultations. $20 \mathrm{We}$ would like to suggest emphatically that this kind of research will also have to be focussed on the reasons why doctors perform as they do, for example by asking doctors about the reasons they have for carrying out or not carrying out certain actions during a consultation. Another way to find out whether doctors have good reasons to perform as they do in real practice is to compare the findings on the content of consultations with findings in the literature about the medical content of such consultations. It is sad that most of the current research (at least in the Netherlands) with regard to standards 
is based mainly on questionnaires to doctors, asking whether they support the standards programme and whether they are aware of the content of standards. This type of research has serious shortcomings, as we have showed in this study. 21 .

As long as research with regards to standards is still in its infancy, we would like to state that when it comes to the assessment of actual care for licensure reasons, current standards cannot yet be used.

More research should also be addressed to the hypothesis that the implementation of standards in actual practice indeed leads to a better outcome for patients, because in the end the only good reason for working according to standards for general practitioners will have to be that it produces better outcomes.

\section{References}

1. Senior JR. Towards the measurement of competence of medicine. Philadelphia: National Board of Medical Examiners 1976.

2. Lloyd JS. Definitions of competence in specialties of medicine. Chicago: American Board of Medical specialties 1979.

3. Viz. Stillman PL. Personal communication.

4. Cate ten RS. Simulatie 1. en Verbrugh HS. Simulatie 2. Letters to the Editor. Huisarts Wet $1988 ; 31: 141-2$.

5. Rethans JJE. Standards for primary care. (Letter to the editor). I Fam Prac 1988; 26: 248-9.

6. Vossen JJJH. 'Modern Times', Letter to the Editor. Med Contact 1991; 46; 133-4.

7. Watts G. Medicine Now. Standardized patients in real practice. British Broadcast Corperation 4; March 12 1991: 11.00-11.10 am.

8. Rethans J. Validity of case simulations. Letter to the Editor. J Clin Epidemiol; in press.

9. Stillman PL. Ruggil JS, Rutala PJ, Sabers DL. Patient instructors as teachers and evaluators. J Med Educ 1980; 55: 186 93.

10. Stillman P, Ruggill J, Rutala P,Sabers D. An instructional program using patient instructors as teachers and evaluators. Proc Annu Conf Res Med Educ 1979; 18: $53-8$.

11. Stillman PL, Ruggill JS, Rutala PJ, Sabers DL. Patient instructors as teachers and evaluators. J Med Educ 1980; 55: 186-93.

12. Schmidt HG, Norman GR, Boshuijzen HPA. A cognitive perspective on medical expertise: Theory and implications. Acad Med 1990; 65:611-21.

13. Schmidt HG, Boshuijzen HPA, Hobus PPM. Transitory stages in the development of medical expertise: the intermediate effect in clinical case representation studies. In Proc. 10th Conf Cognitive Science Soc. Hillsdale, New Jersey: Erlbaum; 1988: 139-45.

14. Norman G, Allery 1, Berkson L et al. Research in the psychology of clinical reasoning: implications for assessment. In: Jolly $B$ (ed). New directions in the assessment of clinical competence. Proc Cambridge Conf IV, Madingley Hall, Cambridge. In press.

15. Kundel HL, Nodine CF, Carmody D. Visual scanning, pattern recognition and decision making in pulmonary nodule detection. Invest. Radiol. 1987; 13: 175-81.

16. Hobus PPM, Schmidt HG, Boshuizen HPA, Patel VC. Contextual factors in the activation of first hypotheses: expert-novice differences. Med Educ 1987; 21: 471-76.

17. Vleuten van der CPM, Verwijnen GM. A system for student assessment. In: Vleuten van der $C$, Wijnen van W (eds). Problem-based learning. Perspectives from the Maastricht Experience. Amsterdam 1990; Thesis Publishers.

18. Hobus PPM, Hofstra ML, Boshuizen HPA, Schmidt HG. De context van de klacht als diagnosticum. Huisarts Wet 1988; 31:261-67. 
19. Neufeld VR, Norman GR. A ssessing clinical competence. Springer Publishing Company, New York. 1985.

20. Pringle M, Stewart-Evans $C$. Does the awareness of being video recorded affect doctors consultation behaviour? Br J Gen Pract 1990; 40: 455-8.

21. Rethans JJE, Van Boven CPA. Simulated patients in general practice: a different look at the consultation. $\mathrm{Br}$ Med J 1987; 294: 809-12. 



\section{Summary}

The introduction of this book (chapter 1) provides the reader with the reasons why the studies, described here, have been conducted.

The immediate instigation was the finding in literature that there might be a discrepancy between what doctors do in their practice and what they tell you they do in an interview or in a questionnaire. It is also described why there were reasons to hypothesize that doctors can do better in a test situation than in actual practice. These questions called for a method, which was able to establish the performance in real practice of general practitioners. It is described why the method of standardized patients was chosen for our studies. Reasons are given for the definitions used in this book, i.e. performance is "what a doctors does in his day-to-day practice" and competence is "what a doctor is capable of doing".

Two main purposes are described. The first was to investigate whether it was possible for a set of standardized (or simulated) patients to visit general practitioners during normal surgery hours, without being detected. The second purpose was to investigate 1) the difference and 2) the relationship between competence and performance of general practitioners.

Chapter 2 presents the results of the first experiment with standardized patients in actual practice. Standardized patients made appointments with 48 general practitioners during actual surgery hours and collected facts about their performances. The standardized patients were indistinguishable from real patients and presented a standardized story of a symptomatic urinary tract infection. Two months later the same general practitioners received a written simulation about a patient who had the same urinary tract infection and were asked how they would handle this problemin real practice. Both results were scored against an existing consensus standard.

The overall score for both methods did not show any substantial differences. A more differentiated analysis, however, showed that general practitioners performed significantly better with standardized patients. It is also showed that general practitioners answering the written simulation performed significantly more superfluous actions. The results of this experiment show that the use of standardized patients seems to show the efficient performance of general practitioners.

Chapter 3 presents the results of the same experiment as was described in chapter 2, but now focussing on the feasibility of sending standardized patients into doctors offices. This chapter also provides the reader with the actions, as they were performed by the general practitioners in real practice. These data were scored according to a consensus standard. It is concluded that the method of introducing standardized patients into doctors offices was 
feasible and that the participating physicians performed only $60 \%$ of the actions, which were defined to be essential in the standard used. These results are discussed.

Chapter 4 presents a review on the use of standardized patients. Main purpose of this chapter was to search for the purposes for which standardized patients have been used. Also the feasibility, reliability and validity of the use of standardized patients was investigated.

It is concluded that for educational purposes standardized patients are a unique method and that the validity of the method is good. Concerning the reliability of the use of standardized patients for educational purposes, it is concluded that research on reliability has only recently started and that the results are promising, but more research has to be conducted. The use of standardized patients for practice purposes shows promising results on reliability and validity assesment.

Chapter $\mathbf{5}$, a review paper based on the 18 most important studies in the literature about medical competence, tries to re-initiate the debate about "what is a competent general practitioner?" by proposing clear distinction between 'competence', (what a physician is capable of doing) and 'performance' (what a physician does in his day-to-day practice). With this distinction we looked at whether studies defined both competence and performance, how they dealt with these concepts, what measurement instruments were used and what the conclusion of the studies were.

Although it is the common reasoning that competence is a good predictor of performance, this concept could not be affirmed. The survey showed that the majority of studies use wrong concepts and come to invalid conclusions. With the empirical distinction between competence and performance however, this chapter proposed new directions for quality assessment of general practitioners.

Chapter 6 describes in detail the method to introduce standardized patients into general practice consultations. The chapter looked first at the general preparation by which the method was organized regardless of individual practices or doctors and secondly at the specific preparation concerning the fine detail of the individual visit. The method was tested in 156 consultations with 39 general practitioners and in no cases were the standardized patients detected. It was concluded that the standardized patient method, following the step-by-step procedure described, is feasible in actual practice.

Chapter 7 represents the results of a study in which 39 general practitioners were each visited during normal surgery hours by four standardized patients. The objective of the study was to see whether actual 
performance of general practitioners, as assessed by standardized patients, met predetermined consensus standards of care for actual practice.

The mean group scores of the doctors on the standards ranged from 33 to $68 \%$. The results show that standardized patients may be the method of choice in the assessment of the quality of actual care of doctors. It is hypothesized that the substandard scores of the doctors do not reflect inadequate competence, but are a result of the difference between competence and performance.

Chapter 8 shows the results of a study, which purposes it was to investigate 1) the difference and 2) the relationship between performance and competence. 39 Family physicians were consulted by four, incognito, standardized patients, portraying four different cases, during normal surgery hours. Later 34 of the 39 doctors participated in a controlled practice test at the Medical School, for which they were asked to act to the best of their ability. Here they were confronted with exactly the same standardized cases (different patients) as they had seen earlier in real practice. Based on the reports of the standardized patients, scores were assigned to the physicians.

Results showed that mean competence score of the physicians was substantially higher than their mean performance score. The Pearson correlation between the competence score and the performance score of the participating physicians was negligable. However, when efficiency and consultation time of the consultations were taken into account, moderate to high correlations were found. It was concluded that performance and competence should be considered as distinct constructs. Assessment of competence under examination circumstances can only have predictive value for performance in actual practice when factors such as efficiency and consultation time are taken into account. Below standard performance of physicians does not necessarily reflect a lack of competence.

Chapter 9 rehearses the main conclusions of the research described in this book.

With respect to the method of standardized patients in real practice, it is concluded that this method has proved to be feasible and reiiable. For further research with standardized patients in real practice it would be worthwhile to extend the reports of standardized patients also to the social and attitudinal skills of doctors. It is also adviced to use the method for the study of interdoctor variation.

Concerning the relationship between competence and performance it is concluded that physicians are able to perform more actions in a competence setting compared with the performance setting. On the other hand if efficiency is taken into account, physicians do better in real practice than in a test setting. It is concluded that competence and performance should be considerd as two distinct constructs. 
With regard to the issue whether competence is a predictor of performance, it appears to be crucial to choose the correct variable to measure the correlation between competence and performance. It is concluded that the factor 'time' seems to play a major role in this. It is adviced that more research is necessary to be able to estimate the importance of this time factor.

Concerning standards of care it is concluded that the participating physicians performed below standards in the performance part. On the other hand, they performed considerably better in the competence part, but still below standards of care. More research is necessary to study the validity of standards. At present standards are not (yet) an instrument to assess the quality of pertormance of physicians in real practice. For educational purposes, however, they are good material. 


\section{Samenvatring}

De introductie van dit boek (hoofdstuk 1) bevat de redenen voor het beschreven onderzoek. Aanleiding tot dit onderzoek was een literatuurreferentie, die de hypothese bevatte dat er een verschil bestaat tussen wat artsen in hun dagelijkse praktijk doen, en wat ze in interviews of in antwoord op een vragenlijst zeggen te doen. Daarnaast geeft de introductie de achtergronden van de hypothese dat artsen in een testsituatie beter kunnen handelen dan in de dagelijkse praktijk. Beide hypothesen vereisten een onderzoeksmethode die het feitelijk handelen (performance) van artsen kon vastleggen. Hier wordt beschreven waarom de simulatiepatiënt-methode hiervoor gekozen is.

Tevens bevat de introductie een verantwoording van de gehanteerde definities van de begrippen 'performance' en 'competence' (resp. 'dat wat een arts doet in zijn dagelijks praktijk-handelen' en 'dat waar een arts toe in staat is').

Ten slotte worden de twee hoofddoelen van het onderzoek aangegeven. Het eerste was te onderzoeken of het voor simulatiepatiënten mogelijk was, om huisartsen tijdens hun dagelijkse spreekuren onontdekt te bezoeken. Het tweede doel was, 1) het verschil, en 2) de relatie tussen competence en performance van huisartsen te onderzoeken.

Hoofdstuk 2 vermeldt de resultaten van het eerste experiment met simulatiepatiënten in de feitelijke praktijk. Deze simulatiepatiënten maakten afspraken bij 48 huisartsen, en verzamelden gegevens over deze echte consulten. Deze patiënten waren getraind in het spelen van een gestandlaardiseerde rol: die van een vrouw met een symptomatische urineweginfectie. Twee maanden na deze bezoeken ontvingen de bezochte artsen een schriftelijke simulatiepatiënt-casus met dezelfde inhoud als tijdens de eerdere praktijkbezoeken. Daarbij kregen zij de vraag voorgelegd hoe ze met een dergelijke patiënt in de praktijk zouden omgaan. Hun handelen werd gescoord volgens een consensus-standaard.

Een globale analyse lijkt geen verschil tussen deze twee methoden aan te geven. Een gedifferentieerde analyse geeft echter aan dat de deelnemende artsen het tijdens het echte consult significant beter deden dan op schrift. Op het punt van niet-noodzakelijke en overbodige handelingen verrichtten de artsen meer handelingen op schrift dan in werkelijkheid. De resultaten geven aanleiding, een efficiënt spreekuurgedrag van artsen te veronderstellen.

Hooldstuk 3 geeft andere resultaten weer van het in hoofdstuk 2 besproken onderzoek, - nu met de nadruk zowel op de uitvoerbaarheid van het gebruiken van simulatiepatiënten voor praktijkonderzoek, als op de in de praktijk verzamelde gegevens. Dit leidt tot de conclusie dat de 
simulatiepatiënt-methode bruikbaar lijkt om data tijdens spreekuren te verzamelen. Tevens blijkt dat de huisartsen slechts $60 \%$ van de in de standaard als noodzakelijk gedefinieerde handelingen verrichtten. Deze resultaten worden bediscussieerd.

Hoofdstuk 4 is een literatuuronderzoek naar de doeleinden waarvoor simulatiepatiënten door de jaren heen zijn gebruikt, en naar de betrouwbaarheid en de validiteit van dit gebruik. De conclusie luidt dat de simulatiepatiënt-methode voor educatieve doeleinden een uniek leermiddel is gebleken en dat de validiteit goed is. Betreffende de betrouwbaarheid van simulatiepatiënten voor educatieve doeleinden valt te constateren dat de betreffende resultaten veelbelovend zijn, maar dat hiergaar meer onderzoek nodig is. Het gebruik van simulatiepatiënten voor praktijkdoeleinden is op dit punt beter onderzocht en de resultaten zijn goed.

Hoofdstuk $\mathbf{5}$ is een literatuuronderzoek, gebaseerd op de achttien belangrijkste studies naar medische competentie. Hiervoor is onderscheid gemaakt tussen 'performance' (dat wat een arts doet in zijn dagelijks praktijk-handelen) en 'competence' (dat waartoe een arts in staat is). Met dit onderscheid zijn de achttien studies bekeken op de vragen : of, en zo ja, hoe zij deze begrippen definiëren; welke meetinstrumenten gebruikt zijn; en wat de conclusies van de studies zijn.

Hoewel algemeen de gedachte heerst dat competence een goede voorspeller van performance is, wordt deze gedachte niet bevestigd. Het onderzoek toont aan dat de meerderheid van de onderzochte studies de verkeerde begrippen gebruikt en niet-valide conclusies trekt. Ter afsluiting geeft het hoofdstuk nieuwe wegen aan om de kwaliteit van huisartsgeneeskundig handelen vast te stellen.

Hoofstuk 6 beschrijft gedetailleerd de stappen die nodig zijn om simulatiepatiënten een bezoek te laten brengen aan huisartsen in de echle praktijk. Eerst wordt de algemene voorbereiding beschreven, los van kenmerken van individuele praktijken. Dan volgt een beschrijving van de specifieke voorbereiding, die van praktijk tot praktijk verschilt.

Deze methodiek is getest in 156 consulten bij 39 huisartsen, waarbij geen enkele simulatiepatiënt ontdekt is. De conclusie is dat de simulatiepatiënt ${ }^{-}$ methode voor gebruik tijdens echte spreekuren, volgens de beschreven gefaseerde aanpak, zeer geschikt is.

Hoofdstuk 7 geeft de resultaten weer van een onderzoek waarin 39 huisartsen, tijdens hun spreekuren ieder door vier verschillende simulatiepatiënten werden bezocht. Het doel hiervan was, te onderzoeken hoe artsen in hun dagelijkse praktijk handelen ten opzichte van vastgestelde consensus-standaarden. 
Het blijkt dat artsen per standaard gemiddeld 33 tot $68 \%$ van de handelingen verrichten die volgens de standaarden obligaat zijn. Dit leidt tot de hypothese dat het niet volgens standaarden werken geen gevolg is van onvoldoende competentie, maar eerder het gevolg is van een verschil tussen competence en performance.

Hoofdstuk 8 bespreekt de resultaten van een onderzoek dat tot doel had, 1) het verschil en 2) de relatie tussen competence en performance van huisartsen te onderzoeken. Hiertoe werden 39 huisartsen tijdens hun spreekuren bezocht door vier simulatiepatiënten, die elk een andere klacht naar voren brachten. Deze simulatiepatiënten waren als zodanig niet te herkennen. Hierna deden 34 van de 39 huisartsen mee aan een gecontroleerde test op het skillslab van de universiteit, waarbij de artsen het specifieke verzoek kregen, kwalitatief zo hoog mogelijk te handelen. Tijdens deze test werden de artsen geconfronteerd met simulatiepatiënten met dezelfde casuïstiek als de artsen eerder in de praktijk was aangeboden. Op grond van de rapportages van de simulatiepatiënten zijn per arts scores berekend.

De resultaten geven aan dat de gemiddelde competence-score van de artsen significant hoger is dan de gemiddelde performance-score. De Pearsoncorrelatie tussen de competence- en de performance-score is verwaarloosbaar laag. Doch bij verdiscontering van de efficiëntie en de consultduur in de scores zijn goede tot hoge correlaties te vinden.

De conclusie luidt dat competence en performance als twee verschillende begrippen moeten worden beschouwd. Het meten van competentie onder examen-omstandigheden heeft alleen voorspellende waarde voor de dagelijkse praktijk, als efficiëntie en consultduur ook medebepaald worden. Het niet volgens standaarden handelen hoeft geen gebrek aan competentie te betekenen.

Hoofdstuk 9 herhaalt de belangrijkste conclusies van het onderzoek.

Wat de simulatiepatiënt-methode voor gebruik in de dagelijkse praktijk betreft, luidt de conclusie dat deze methode bruikbaar, betrouwbaar en valide is. Aanbevolen wordt, meer onderzoek te doen met simulatiepatiënten in de dagelijkse dokterspraktijk, zowel naar het rapporteren van sociale vaardigheden en attitude-vaardigheden, als naar interdokter-variatie.

Betreffende de verhouding tussen competence en performance is de conclusie dat artsen meer handelingen verrichten in een competence-situatie dan in een performance-situatie. Als echter ook naar efficiëntie wordt gekeken, doen artsen het beter in de praktijk dan in de testsituatie. De gevolgtrekking is dat competence en performance als twee verschillende begrippen moeten worden beschouwd.

Inzake de schatting van de correlatie tussen competentie en praktijkhandelen, blijkt dat het van wezenlijk belang is, hiervoor de juiste variabele 
te kiezen. De conchusie op dit punt is dat de consultduur een belangrijke rol speelt en dat meer onderzoek hiernaar nodig is.

Tevens is geconcludeerd dat artsen in hun dagelijks praktijk-handelen niet volgens standaarden handelen. In een testsituatie doen ze het wel beter, maar nog steeds niet volgens de standaarden. Er is meer onderzoek naar de validiteit van standaarden nodig, voordat deze bruikbaar zijn als maat voor de kwaliteit van handelen. Voor onderwijskundige doeleinden zijn standaarden echter wel een goed leermiddel. 
Innledningen i denne boken (kapittel 1) behandler bakgrunnen for den beskrevne undersøkelsen.

Foranledningen til denne undersøkelsen var en litteraturhenvisning, som inneholdt den hypotese at det er en forskjell på hva leger foretar seg i sin daglige prakis og hwa de sier i intervjuer eller i sine svar på spørreskjemaer, at de foretar seg. Dessuten gir innledningen bakgrunnene for den hypotese at leger i en forsøkssituasjon kan handle bedre enn i den daglige praksis. Begge hypoteser krevde en undersøkelsesmetode som kunne registrere legers faktiske fremgangsmåte ('performance'). Her beskrives hvorfor en undersøkelsesmetode ved bruk av simulerte pasienter er valgt til dette formålet.

Videre inneholder innledningen en redegjørelse for de brukte definisjoner av begrepene 'performance' og 'competence' (hhv. 'det som en lege gjør i sin daglige yrkesmessige virksomhet' og 'det som en lege er i stand til å gjøre').

Til sist nevnes undersøkelsens to hovedformål. Det første var å undersøke om det var mulig for simulerte pasienter å besøke praktiserende leger i deres daglige konsultasjonstid uten å bli oppdaget. Det andre formål var å undersøke 1) forskjellen, og 2) forholdet, mellom praktiserende legers 'competence' og 'performance'.

Kapittel 2 nevner resultatene av det første eksperimentet med simulerte pasienter i den faktiske praksisen. Disse simulerte pasientene fikk avtaler hos 48 praktiserende leger, og samlet inn data om disse faktiske konsultasjonene. Disse pasientene hadde blitt lært opp til å spille en standardisert rolle: som en kvinne med en symptomatisk urinveisinfeksjon. To måneder etter disse besøkene mottok de konsulterte leger et skriftlig kasus med det samme innholdet som under de tidligere faktiske konsultasjonene med de simulerte pasientene. I denne forbindelse ble de spurt om a beskrive hwordan de ville behandle en slik pasient i praksis. Deres handlemăte ble målt etter en konsensus-standard.

En grov analyse synes ikke å vise noen forskjell på disse to metodene. En differensiert analyse viser imidlertid at de deltakende legene handlet signifikant bedre under den virkelige konsultasjonen enn i sine skriflige svar. Med hensyn til unødvendige og overfladige handlinger skrev legene at de utforte flere av disse enn det viste seg at de gjorde $\mathrm{i}$ virkeligheten.

Resultatene gir grunn til å anta en effektiv handlemăte $\mathrm{i}$ konsultasjonstiden.

Kapittel 3 giengir andre resultater av undersøkelsen diskutert i kapitel 2, denne gangen både når det gjelder muligheten til å gjennomføre bruk av simulerte pasienter til prakis-undersøkelser, og når det gjelder de faktisk 
innsamlede data. Dette forer til den konklusjon at metoden med simulerte pasienter synes å kunne brukes til innsamlingen av data i konsultasjonstiden. Desuten viser det seg at de praktiserende legene utferer bare $60 \%$ av de handlinger som i standarden defineres som nødvendige. Disse resultater diskuteres.

Kapittel 4 er en litteraturundersøkelse av de formălene simulerte pasienter har vart benyttet til i årenes lop, og av påliteligheten og validiteten av å bruke simulerte pasienter.

Konklusjonen er at bruk av simulerte pasienter til undervisningsformål har vist seg å være et enestående læremiddel og at validiteten er god. Med hensyn till påliteligheten av simulerte pasienter til undervisningsformål kan det fastslås at de aktuelle resultatene er lovende, men at det er nodvendig med ytterligere forskning på dette område. Bruk av simulerte pasienter til praktiske formăl er blitt bedre undersøkt på dette punkt, og resultatene er gode.

Kapittel 5 er en litteraturundersøkelse, som er basert på de atten viktigste studier i lege-kompetanse.

I denne forbindelse skjelnes det mellom 'performance' (det som en lege giør i sin daglige yrkesmessige virksomhet) og 'competence' (det som en lege er i stand til ẩ gjøre).

På bakgrunn av denne forksjellen er de atten studier blitt undersøkt med hensyn til følgende spørsmål: om, og i så fall, hvordan, de definerer disse begrepene; hvilke måleinstrumentene som er blitt brukt; og hvilke konklusjoner studiene trekker. Skjønt det er en generell oppfatning at 'competence' gir en god forutsigelse av 'performance', bekreftes denne oppfatningen ikke. Undersøkelsen viser at de fleste studier bruker uriktige begreper og trekker ikke-valide konklusjoner.

Til slutt viser kapitlet nye veier til å fastslå kvaliteten av legers fremgangsmåte.

Kapittel 6 gir en detaljert beskrivelse av de trinn som er nodvendige for a fă simulerte pasienter til å konsultere praktiserende leger i virkelig praksis.

Forst beskrives den alminnelige forberedelsen, uten hensyn til kjennetegn av individuelle legers praksis. Deretter folger en beskrivelse av den spesifikke forberedelsen, som er forskjellig fra praksis til praksis. Denne metodikken er blitt provd i 156 konsultasjoner hos 39 praktiserende leger, og ingen simulert pasient er blitt oppdaget.

Konklusjonen er at metoden med simulerte pasienter, til bruk under virkelige konsultasjoner, etter den beskrevne trinnvise metoden, egner seg meget godt. 
Kapittel 7 gjengir resultatene av en undersøkelse der 39 praktiserende leger hver ble besøkt av fire forskjellige simulerte pasienter i konsultasjonstiden.

Formålet med dette var 3 undersøke hvordan leger i sin daglige praksis handler sammenlignet med faste konsensus-standarder. Det viser seg at leger pr. standard utfører i gjennomsnit 33 til $68 \%$ av de handlingene som ifølge standardene er obligatoriske.

Dette fører til den hypotese at avvikelsen fra standarden ikke er en følge av utilstrekkelig kompetanse, men snarere en følge av en forskjell på 'competence' og 'performance'.

Kapittel 8 diskuterer resultatene av en undersøkelse met det formål, å undersøke 1) forskjellen, og 2) forholdet, mellom praktiserende legers 'competence' og 'performance'.

I denne forbindelse ble 39 praktiserende leger besøkt av fire simulerte pasienter, som hver for seg kom med forskjellige symptomer. Disse simulerte pasientene var ikke til å gjenkjenne som sådan. Heretter deltok 34 av de 39 praktiserende leger i en kontrollert prove i ferdighetslaboratoriet på universitetet, der de uttrykkelig ble spurt om å handle kvalitativt høyest mulig. Under denne proven ble legene konfrontert med simulerte pasienter med de samme symptomene som legene tidligere var blitt konfrontert med i sin praksis. På grunn av de simulerte pasienters rapporter er det blitt beregnet poengsum pr. lege.

Resultatene viser at legenes 'competence'-score i gjennomsnitt er signifikant høyere en den gjennomsnitlige 'performance'-score. Pearsonkorrelasjonen mellom competence'- og 'performance'-scoren er ubetydelig lav. Men dersom effektiviteten og konsultasjonsvarigheten $i$ disse 'scores' tas i betraktning, finnes det fra gode til hoye korrelasjoner.

Konklusjonen er at 'competence' og 'performance' má betraktes som to forskjellige begrep. Måling av kompetanse under eksamensomstendigheter har bare forutsigelsesverdi for den daglige praksis, når effektiviteten og konsultasjonsvarigheten også tas i betraktning. Det at legen ikke handler etter standarder behøver ikke å bety mangel på kompetanse.

Kapittel 9 gjentar undersøkelsens viktigste konklusjoner.

Vedrørende undersøkelsesmetoden med simulerte pasienter til bruk i den daglige prakis, er konklusjonen at denne metoden er formålstjenlig, pålitelig og valid. Det anbefales å forske mer med simulerte pasienter i den daglige praksis, både hva angår rapportering av sosiale ferdigheter/holdningsferdigheter, og lege-variasjon.

Med hensyn til forholdet mellom 'competence' og 'performance' er konklusjonen at leger utforer flere handlinger i en 'competence'-situasjon enn i en 'performance'-situasjon. Tas imidlertid også effektiviteten i betraktning, handler leger bedre i praksis enn i forsøkssituasjonen. 
Konklusjonen er at 'competence' og 'performance' må betraktes som to forskjeligge begrep.

Hva angằ vurderingen av korrelasjonen mellom kompetanse og praktisk handling viser det seg à vare av vesentlig betydning à velge den rette variabelen. Konklusjonen på đette punktet er at konsultasjonsvarigheter spiller en viktig rolle og at ytterligere forskning på dette omrädet er nødvendig.

En anden konklusjon er at leger i sin daglige praksis ikke handler etter standarder. I en forsøkssituasjon gjør de det riktignok bedre, men likevel ikke etter standardene.

Ytterligere forskning av standarders validitet er nødvendig, for disse lar seg bruke til å måle handlingskvaliteten. Til undervisningsformål er standarder imidlertid et godt laremiddel. 


\section{Thanks}

Successful research depends on many people.

I have asked for and received the help and advice of a considerable number of persons. Many of them work in the field of research, others in the field of general practice. I am also most grateful for the assistance and support which I have received from quite a number of people outside the academic world, such as my family and friends.

This study would have been impossible without the participating general practitioners, whose names I have promised not to mention, and without the help and advice of Ferd Sturmans, Riet Drop, Cees van der Vleuten, Trudie Seegers and Geoff Norman (McMaster University, Hamilton, Canada).

Dear friends, I would like to thank you all for helping me during the stressful and exciting, but at the same time very pleasant years that are now behind me. I hope that it will be possible to continue to cooperate with many of you in the future.

I would like to mention (in alphabetical order):

Pierre Bastings, Els Boshuizen, Els Bour, Cees van Boven, Dhr. Braams, Pie Castermans, Michiel Cornel, Donald Crombie (Birmingham, UK), Pia Cuijpers, Dhr. van Deelen, Geert-Jan Dinant, Frans Duijsens, Yvonne L'Espoir-Dohmen, Toon van Gerven, Gale Glivia (McMaster University, Hamilton, Canada), Erik de Graaf, Diederik Grit, Richard Grol, Bea de Groote, Theo Hellemons, Leon Heuts, Pie Hobus, Ron Hoogenboom, Paul Höppener, Robin Hull (Birmingham, UK), Caroliene Janssens, Jan-Willem de Jonge, Victor Kaiser, Juul Kerbusch, Piet Kerkhof, Jan Klerkx, Andre Knottnerus, Peter Kramer, Marike Laning, Yvonne van Leeuwen, Jan Leewis, Martha Lucassen, Wil Macco, Jaap Metz, Jean Muris, Annemarie Muysken, Paul Nekeman, Frank van Onna, Charles Phaff, Marijke Perquin, Piet Portegijs, Pieter Ramler, Jan C.A. Rethans, Thea Rethans-Willems, Henk Schmidt, the sickfund SVGZ in Maastricht (Board of Directors and Dhr J. Schoffelen in particular), the sickfund LIASS in Heerlen and Sittard (Board of Directors and Dhr J. Quadvlieg and Dhr. Rohs in particular), Richard Starmans, Jelle Stoffers, Jacques van Thiel, Marijke Verdonk, Theo Voorn, Maarten Verwijnen, George Wolfs, Wim van Zutphen ànd Paul Zwietering. 


\section{Curriculum vitae}

Jan-Joost Rethans was born on January 11 th, 1955 in Delft, the Netherlands. He finished his secondary education (grammar school, specializing in sciences) at St. Stanislas' College in Delft in 1973. From 1973 to 1974 he studied economics at the Erasmus University in Rotterdam and from 1974 to 1975 he was a student at the medical school of the University of Ghent, Belgium.

From 1975 to 1982 he studied medicine at the University of Limburg in Maastricht. He spent the year1980-1981 in Grindaheim, Norway, working in general and veterinarian practice in a rural area. He graduated from the University of Limburg in 1982, remaining at the same university to start training as a general practitioner. In the period from 1983 to 1984 he was a general practitioner in several practices in the Netherlands. In 1984 he worked at the psychiatric hospital in Reinsvoll, Norway.

From 1985 to 1986 he participated the one-year Research Training Course for general practitioners at the University of Limburg.

Since 1986 he has been working as a researcher/general practitioner at the Department of General Practice of the University of Limburg, where he carried out the studies presented here. To learn more about standardized patients he spent five weeks (in 1986) at the Department of Clinical Epidemiology \& Biostatistics of McMaster University in Hamilton (Canada), where he worked under the supervision of Prof. G.R. Norman.

Since 1987 he has been a member of the Executive Board of the European General Practice Research Workshop (EGPRW).

$\mathrm{He}$ is married with four children. 


\section{Appendices}

1. Detection form for the physicians to report standardized patients.

2. Instructions for the participating general practitioners (competence setting)

3. The standards of care used 


\section{Detection form to report standardized patients}

Date:

Name physician:

Address:

Postal Code:

City:

On what date did you detect the standardized patient?

What name did the standardized patient use to present him/herself?

Was the standardized patient a male/female?

For what main complaint did the standardized patients ask your help?

What reasons made you think that this was a standardized patient?

Did you detect the standardized patient before/during/after the consultation?

Do you believe that the detection of the standardized patient had a serious impact on your performance during that consultation?

Would you please encircle how certain you are that the patient, whom you are now reporting to us, is indeed a standardized patient?

Absolutely certain/ Certain/ Uncertain/ Absolutely uncertain

Further remarks?

Thank you for your cooperation. 

setting)

\section{Dear colleague,}

During the next two hours you will be confronted with one or more standardized patients. You have seen none of these standardized patients before. The problems for which the standardized patients are asking your help differ from the problems presented to you by the standardized patients during their visits in your actual practice. (If the problems were the same ones, you would now recognize them.)

\section{What is the purpose of this setting?}

It has often been assumed that general practitioners deliver sub-optimal patient care in their practice because they do not know how to do better. The purpose of this study is to investigate if that is true. We believe that general practitioners are quite capable of treating their patients well, but that normal practice circumstances (busy surgery hours, telephone calls during contacts, etc) may prevent them providing optimal care.

\section{Top quality}

We would like you to consider the room in which you are now sitting as your own surgery. We would also like you to treat the standardized patient or patients who will be coming to you in a moment with the highest possible degree of quality (anno September 1989). Show the best you are capable of!

We want you to perform to the best of your abilities during this surgery hour. It may be that this will mean that you will be performing better than in your routine surgery hours. We would like to assess the quality of general practitioners' performance when they are working at their top level. This top level performance is required during all parts of a consultation, not only for the history and physical examination but also for the choice of prescription and for concluding the contact.

In this surgery hour you will be free of time pressure. We will assess only the quality of the content of your performance and will not pay attention to the time used. There is no difference for us whether you perform at the same top level of quality in 5 minutes or in 25 minutes. We know from previous experience that much longer than 25 minutes will probably not be neccessary. 


\section{Practical notes.}

From now on, you are a general practitioner in this room. It is morning. If you are used to working in a health centre, then please consider this room as a room in your health centre. If you are used to working in a solo practice, then you are also working in a solo practice now.

\section{Chart sytem}

Each of the patients to come represents a new patient to you. Your medical secretary has already listed this patient in your practice and she has already made up a patient file (medical chart). This will be handed to you by the patient him/herself. You do not have to bother about details such as the visiting standardized patient's previous general practitioner.

We would like to ask you to fill in on this medical chart (during or after the consultation) those items which you consider absolutely necessary. This medical chart should also reflect the top performance of this consulation.

\section{Laboratory examination}

We would like you to complete each consultation. If you wish the patient to go to a hospital laboratory (for a blood test, for example), then please fill in a lab form. The lab forms are in front of you and are the same as the ones you are used to. After handing the form to the patient, you will end the consultation, again in the top-level manner. If, on the other hand, you are used to doing lab tests in your own laboratory, then you may ask the medical student, who will be sitting in this room too, for the result of the test. The result will than be given to you. For example, if you would like to assess the hemoglobin level of the patient or to do a urine test, you may ask the student: "I want to have the hemoglobine value, what is it?" or "I would like to check something in the patient's urine, what are the results?". After having received this information you may continue with the consultation. If you would like to prescribe a drug or drugs to the standardized patient, please write out a prescription and hand it to the patient.

\section{Medical instruments}

We have tried to provide this room with all the usual medical instruments, for instance a stethoscope, a Mercury sphygmomanometer, a 'Snellen' chart, etc. Please take a good look around the room to get acquainted with it. Also take a good look at the instruments. If you think that there is something missing and if you would like to use this device for the examination, then please pretend you are using it and say so clearly to the patient.

In a few moments the first patient will knock on your door. When you have finished the contact, the patient will leave the room. We would like you not to start discussing the contact with the standardized patient afterwards, since he/she has to fill in a form immediately after the contact. 
It is may well be that you will have to wait some time before the next patient enters. Please stay in this room. We have supplied you with a newspaper. If you have urgent questions, please ask the student and he/she will ask me in the corridor.

Once again I would like to thank you for your cooperation in this study.

\section{Good luck!}




\section{History}

1. Obligatory. Time aspects: how long have complaints existed; at what times of the day; how often?

2. Obligatory. Nature of the pain.

3. Obligatory. Location of the pain, radiation, presence of prodomes; progression of complaints.

4. Obligatory. Associated phenomena (such as light phobia, nausea, fever, dental and neck complaints,etc.).

5. Obligatory. Relation with psychosocial circumstances.

6. Intermediate. What is the reaction to the pain.

7. Intermediate. Self therapy.

8. Intermediate. Intoxications (smoking, carbon monoxide).

9. Obligatory. Migraine history (familial history, progression, frequency, connection with circumstances).

10. Superfluous actions history.

\section{Physical examination}

11. Intermediate. Blood pressure reading.

12. Intermediate. Examine eye sight.

13. Intermediate. Examine eye fundus.

14. Intermediate. Cervical spine.

15. Intermediate. Neurological examination.

16. Intermediate. Sinuses: percussion and pressure pain, transillumination.

17. Superfluous actions physical examination.

18. Superfluous actions Laboratory

\section{Guidance and advice}

19. Obligatory. Explain cause of complaints.

20. Obligatory. Discuss prognosis.

21. Obligatory. Explain relationship between complaints and tension.

22. A. Obligatory. In case of therapy: explain expected effect.

B. Obligatory. In case of no therapy: explain why no therapy is prescribed.

23. Intermediate. Relaxation exercises, yoga (brochure).

24. Obligatory. Discuss connection with life style.

25. Superfluous actions guidance and advice.

\section{Therapy}

26. Intermediate. Simple analgetics.

27. Obligatory. Discuss possible background to headache.

28. Intermediate. Benzodiazepines. 
29. Superfluous actions therapy.

\section{Return visit}

30. Obligatory. Indicate whether or not a return visit is necessary, depending on possible increase of complaints and prognosis.

31. Superfluous actions return visit. 
Diagram 2. The standard of the diarrhea case

\section{Mistory}

1. Obligatory. Presence of diarrhea.

2. Obligatory. Presence of nausea, vomiting.

3. Obligatory. Presence of abdominal pain.

4. Obligatory. Course and duration of the complaints.

5. Obligatory. Ask for possible causes such as: contact with illness; travel; special foods, etc.

6. Obligatory. Are there any other complaints?

7. Intermediate. Check medication.

8. Intermediate. Eating habits history.

9. Intermediate. Fever.

10. Obligatory. Stool consistency and frequency.

11. Obligatory. Nature of pain.

12. Obligaat. Location of pain, shifts in location.

13. Superfluous actions history.

\section{Physical examination}

14. Obligatory. Examine abdomen: inspection, percussion, auscultation, palpation.

15. Superfluous actions physical history.

\section{Other laboratory}

16. Obligatory. Stool culture.

17. Superfluous actions other laboratory.

Guidance and advice

18. Obligatory. Present diagnosis.

19. Obligatory. Discuss prognosis.

20. Obligatory. Give diet advice.

21. Superfluous actions guidance and advice.

\section{Medication}

22. Intermediate. Anti-emetics.

23. Intermediate. Adstringents.

24. Superfluous actions medication.

\section{Return visit}

25. Obligatory. Explain that patient should return in two or three days if complaints persist.

26. Superfluous actions return visit. 



\section{History}

1. Obligatory. How long has the complaint existed?

2. Obligatory. Did the pain develop gradually or acutely?

3. Obligatory. Ask location of pain.

4. Obligatory. Can causative moments be found?

5. Obligatory. Is there any movement limitation; when, which movements?

6. Obligatory. How do complaints relate to life and work of patient?

7. Obligatory. Are there any accompanying symptoms such as paresthesias?

8. Intermediate. Ask about self-medication.

9. Intermediate. Are there any general illness symptoms?

10. Superfluous actions history.

\section{Physical examination}

11. Obligatory. Undress above waist, inspection.

12. Obligatory. Ask for pain locations to be indicated.

13. Obligatory. Palpation of pain points.

14. Obligatory. Palpate joint during movement.

15. Obligatory. Check passive movement: extension, flexion, rotation.

16. Obligatory. Check active movement: painful arc, flexed biceps, lower arm extensors.

17. Superfluous actions physical examination.

Guidance and advice

18. Obligatory. Report findings and or any diagnosis.

19. Obligatory. Discuss prognosis.

20. Obligatory. Give movement and exercise advice.

21. Intermediate. Give ergonomic advice.

22. Superfluous actions guidance and advice

\section{Medication and therapy}

23. Intermediate. Sling, immobilisation bandage, splint.

24. Intermediate. Antiphlogistics (NSAID); local corticosteroid injections.

25. Superfluous actions medication and therapy.

\section{Referral}

26. Intermediate. Physiotherapist.

27. Superfluous actions referral. 


\section{Return visit}

28. Obligatory. Indicate whether or not a repeat visit is necessary, in relation with prognosis.

29. Obligatory. Return visit after $2-3$ weeks.

30. Obligatory. Movement sufficiently limited to make working impossible.

31. Superfluous actions return visit. 
Diagram 4. The standard for the diabetes case

\section{History}

1. Obligatory. Ask about health, hypoglycemias.

2. Obligatory. Ask about diet and medication problems.

3. Obligatory. Ask for cause of positive urine reduction $>2 \%$.

4. Obligatory. Pay attention once a year to sight complaints, neuropathy complaints, intermittent claudication complaints.

5. Superfluous actions history.

\section{Physical examination}

6. Obligatory. Determine weight.

7. Obligatory. Take blood pressure.

8. Obligatory. Fundus examination.

9. Obligatory. Inspect feet.

10. Obligatory. Palpation of arteries in feet.

11. Obligatory. Vibration sensee.

12. Superfluous actions physical examination.

\section{Own laboratory}

13. Obligatory. Pre-prandial blood glucose or at fixed time of day.

14. Obligatory. Morning urine protein level.

15. Intermediate. Urine glucose (stick).

16. Intermediate. Basic ECG.

17. Intermediate. Dipslide urine.

18. Intermediate. Determine ketones in urine.

19. Superfluous actions own laboratory.

\section{Other laboratory}

20. Obligatory. Cholesterol and serum lipids.

21. Intermediate. HbAlc.

22. Superfluous actions other laboratory.

Guidance and advice

23. Obligatory. Discuss suggested treatment.

24. Obligatory. Discuss diet.

25. Obligatory. Discuss cause of urine glucose.

26. Obligatory. Point out consequences of overweight.

27. Obligatory. Recommend weight loss.

28. Superfluous actions guidance and advice.

\section{Medication}

29. Obligatory. Discuss treatment policy.

30. Superfluous actions medication. 


\section{Referral}

31. Intermediate. Refer to dietician.

32. Superfluous actions referral.

\section{Return visit}

33. Obligatory. Always schedule new appointment.

34. Obligatory. Return visit after 3-6 months.

35. Superfluous actions return visit. 